

L'Université canadienne Canada's university 
FACULTÉ DES ÉTUDES SUPÉRIEURES ET POSTOCTORALES
IIII

uOttawa
FACULTY OF GRADUATE AND

POSDOCTORAL STUDIES

L.Universite canadienne

Canada's university

Brianna McGrath

AUTEUUR DE LA THESE / AUTTHOR OF THESIS

M.A.Sc. (Biomedical Engineering)

GRADE / DEGREE

Department of Mechanical Engineering

FACULTE, ECOLE, DEPARTEMENT / FACULLTY, SCHOOL, DEPARTMENT

A Mechanobiological Investigation of Platelets

TITRE DE LA THESSE / TITLE OF THESIS

Michel Labrosse

DIRECTEUR (DIRECTRICE) DE LA THESE / THESIS SUPEAVISOR

CO-DIRECTEUAR (CO-DIRECTRICE) DE LA THESE / THESIS CO-SUPERVISOR

EXAMINATEURS (EXAMINATRICES) DE LA THĖSE / THESIS EXAMINERS

James Cheetham

James Harden

Gary W. Slater 


\title{
A Mechanobiological Investigation of Platelets
}

\author{
Brianna McGrath
}

Thesis submitted to the

Faculty of Graduate and Postdoctoral Studies

In partial fulfillment of the requirements

For the MASc degree in Biomedical Engineering

Department of Mechanical Engineering

Faculty of Engineering

University of Ottawa

(CBrianna McGrath, Ottawa, Canada, 2009 




Library and Archives

Canada

Published Heritage

Branch

395 Wellington Street Ottawa ON K1A ON4 Canada
Bibliotheque et

Archives Canada

Direction du

Patrimoine de l'édition

395, rue Wellington

Ottawa ON K1A 0N4

Canada
Your file Votre référence
ISBN: 978-0-494-61167-8
Our file Notre référence
ISBN: $978-0-494-61167-8$

\section{NOTICE:}

The author has granted a nonexclusive license allowing Library and Archives Canada to reproduce, publish, archive, preserve, conserve, communicate to the public by telecommunication or on the Internet, loan, distribute and sell theses worldwide, for commercial or noncommercial purposes, in microform, paper, electronic and/or any other formats.

The author retains copyright ownership and moral rights in this thesis. Neither the thesis nor substantial extracts from it may be printed or otherwise reproduced without the author's permission.
AVIS:

L'auteur a accordé une licence non exclusive permettant à la Bibliothèque et Archives Canada de reproduire, publier, archiver, sauvegarder, conserver, transmettre au public par télécommunication ou par l'Internet, prêter, distribuer et vendre des thèses partout dans le monde, à des fins commerciales ou autres, sur support microforme, papier, électronique et/ou autres formats.

L'auteur conserve la propriété du droit d'auteur et des droits moraux qui protège cette thèse. Ni la thèse ni des extraits substantiels de celle-ci ne doivent être imprimés ou autrement reproduits sans son autorisation.
In compliance with the Canadian Privacy Act some supporting forms may have been removed from this thesis.

While these forms may be included in the document page count, their removal does not represent any loss of content from the thesis.
Conformément à la loi canadienne sur la protection de la vie privée, quelques formulaires secondaires ont été enlevés de cette thèse.

Bien que ces formulaires aient inclus dans la pagination, il n'y aura aucun contenu manquant. 


\section{Table of Contents}

$\begin{array}{ll}\text { Title Page } & \text { i }\end{array}$

Table of Contents $\quad$ ii

List of Tables and Figures iv

Legend

$\begin{array}{lll}\text { Abstract } & \text { vii }\end{array}$

Acknowledgements $\quad$ viii

1 Introduction $\quad 1$

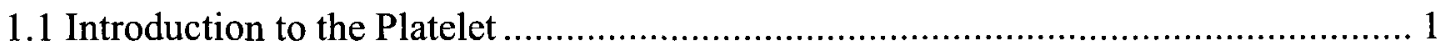





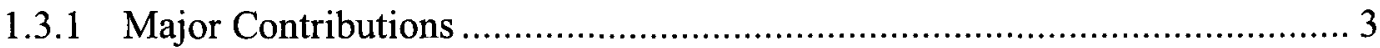



1.4 Organization of Thesis Dissertation.......................................................... 3

2 Background and Literature Review $\quad 5$





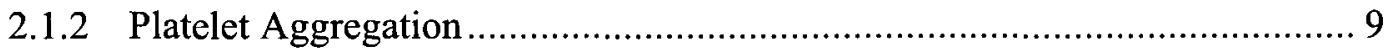

2.1.3 Comparison of Structure and Function between Human and Mouse Platelets ....... 10

2.2 Macroscopic Platelet Biomechanics in the Literature ...................................... 13

2.2.1 Shear-Induced Platelet Activation.................................................... 13

2.2.2 Mechanical Heart Valves and Thrombosis........................................... 17

2.3 Cellular and Subcellular Platelet Biomechanics in the Literature ....................... 18

2.4 Methods to Measure Single Platelets' Mechanical Properties .............................. 20

2.4.1 Atomic Force Microscopy .............................................................. 20

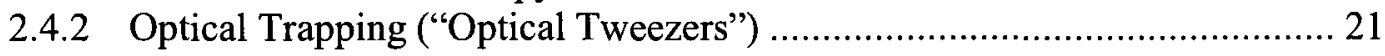





2.5.1 General Principles and Methods in Constitutive Modeling........................ 24

2.5.2 A Constitutive Theory for Biological Membranes.................................... 26

2.5.3 Existing Models Inspired from Erythrocyte Membrane Models ................. 28

3 Experiments $\quad 31$



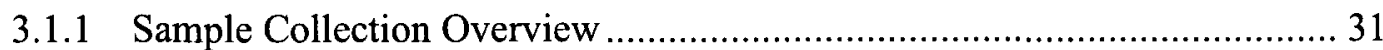




3.1.3 Washed Platelet Preparation .......................................................... 32

3.1.4 Micropipette Aspiration and Imaging Procedure ..................................... 34

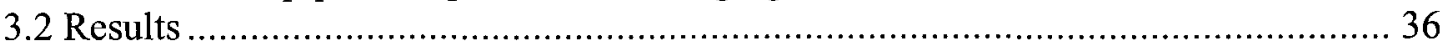

3.2.1 Aspiration Measurements of Inactive Murine Platelet Membranes.............. 36

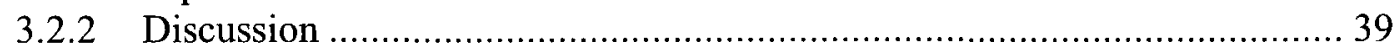

4 Mathematical Model $\quad \mathbf{4 2}$



4.1.1 Geometry of Undeformed Platelet ....................................................... 42

4.1.2 Geometry of Deformed Platelet during Micropipette Aspiration ................. 44

4.2 Equilibrium Equations for a Platelet using Micropipette Aspiration.................... 48



4.4 Problem Formulation and Method of Solution.............................................. 51

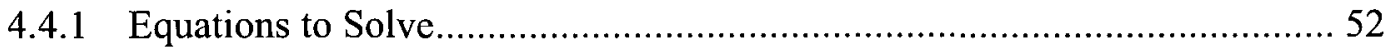

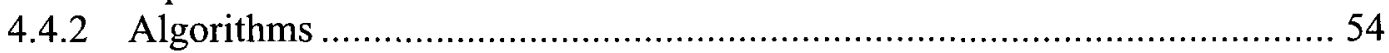















5 Discussion $\quad \mathbf{7 4}$





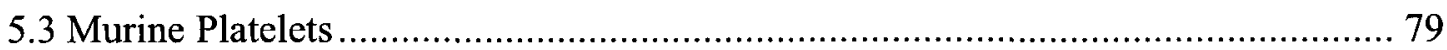

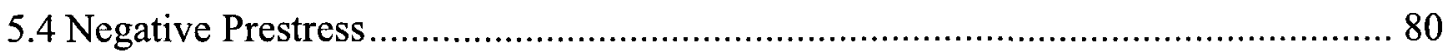



5.6 Limitations of the Current Work and Future Recommendations ......................... 82

6 Conclusions $\quad 84$

Appendix A: Standard Operating Procedure for Rodent Anaesthetization $\quad 86$

Appendix B: Deformed Platelet Assumptions Overview $\quad 90$

Appendix C: Undeformed and Deformed Membrane Elements in Curvilinear
Coordinates

Appendix D: Proof of Equilibrium Equations $\quad 92$

Appendix E: Constitutive Descriptions of Platelet Membrane 95

Appendix F: Zone-dependent Descriptions of Membrane Tension 96

$\begin{array}{ll}\text { Appendix G: MATLAB Files: Commented } & 97\end{array}$

$\begin{array}{ll}\text { Bibliography } & 111\end{array}$ 


\section{List of Tables and Figures}

Tables

2.1: Various functions of organelle-enclosed molecules. 7

2.2: Comparison of platelet parameters in the human and murine species, ........................................... 11

2.3: Amino acid homologies of cloned murine platelet coagulation proteins to human alternatives. .............. 12

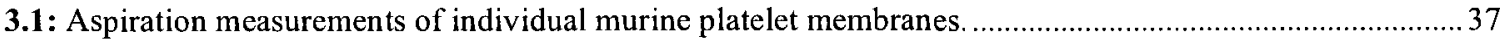

4.1: Material constants for an average $(n=17$ platelets $)$ human platelet membrane $\ldots \ldots \ldots \ldots \ldots \ldots \ldots \ldots \ldots \ldots \ldots . . \ldots 4$



\section{Figures}

2.1: Diagrammatic, cross-sectional representation of the normal human platelet structure with its actin cytoskeletal filaments, and von Willebrand factor (vWF) and fibrinogen from circulating blood plasma.... 6

2.2: Blood flow in a cylindrical chamber of radius $R$ generates a parabolic flow velocity profile 14

2.3: The effect of a stenotic region on the activation of platelets in a circulation loop 16

3.1: Micropipette aspiration of a murine platelet. 36

3.2: Deformation behavior of individual aspirated murine platelet membranes ..... 38

3.3: Average deformation behavior of murine platelets during micropipette aspiration ... 39

4.1: Undeformed platelet geometry 43

4.2: Shell surface geometry for a deformed platelet. 44

4.3: Proposed contour for an aspirated platelet. 45

4.4: Iterative Algorithm to Identify Membrane Material Constants......................................................55

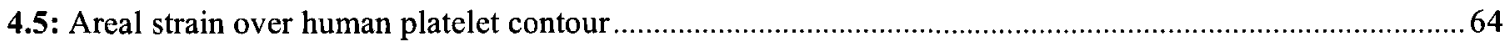

4.6: Average theoretical (x) vs. experimental (o) deformation behavior for the human platelet membrane.... .65

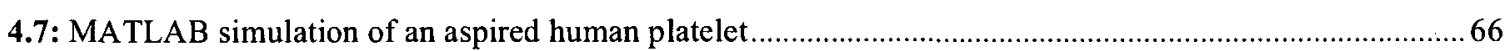

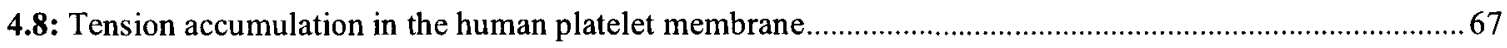

4.9: Longitudinal $\left(\lambda_{s}\right.$, black) and circumferential $\left(\lambda_{\theta}\right.$, grey) stretch ratios over human platelet contour for an

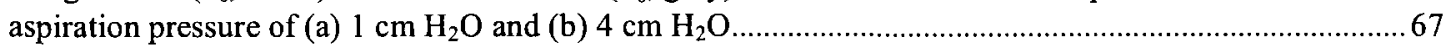

4.10: Areal strain over murine platelet contour 69

4.11: Average theoretical $(x)$ vs. experimental $(0)$ deformation behavior for the murine platelet membrane $\ldots . .70$ 
4.12: MATLAB simulation of an aspired murine platelet.

4.13: Tension accumulation in the murine platelet membrane......

4.14: Longitudinal ( $\lambda_{s}$, black) and circumferential ( $\lambda_{\theta}$, grey) stretch ratios over murine platelet contour for an aspiration pressure of (a) $1 \mathrm{~cm} \mathrm{H} \mathrm{H}_{2} \mathrm{O}$ and (b) $4 \mathrm{~cm} \mathrm{H}_{2} \mathrm{O}$.

C.1: Graphical representation of the initial (undeformed) and final (deformed) geometries of an element on the axisymmetric membrane surface . 


\section{Legend}

\section{List of Symbols}

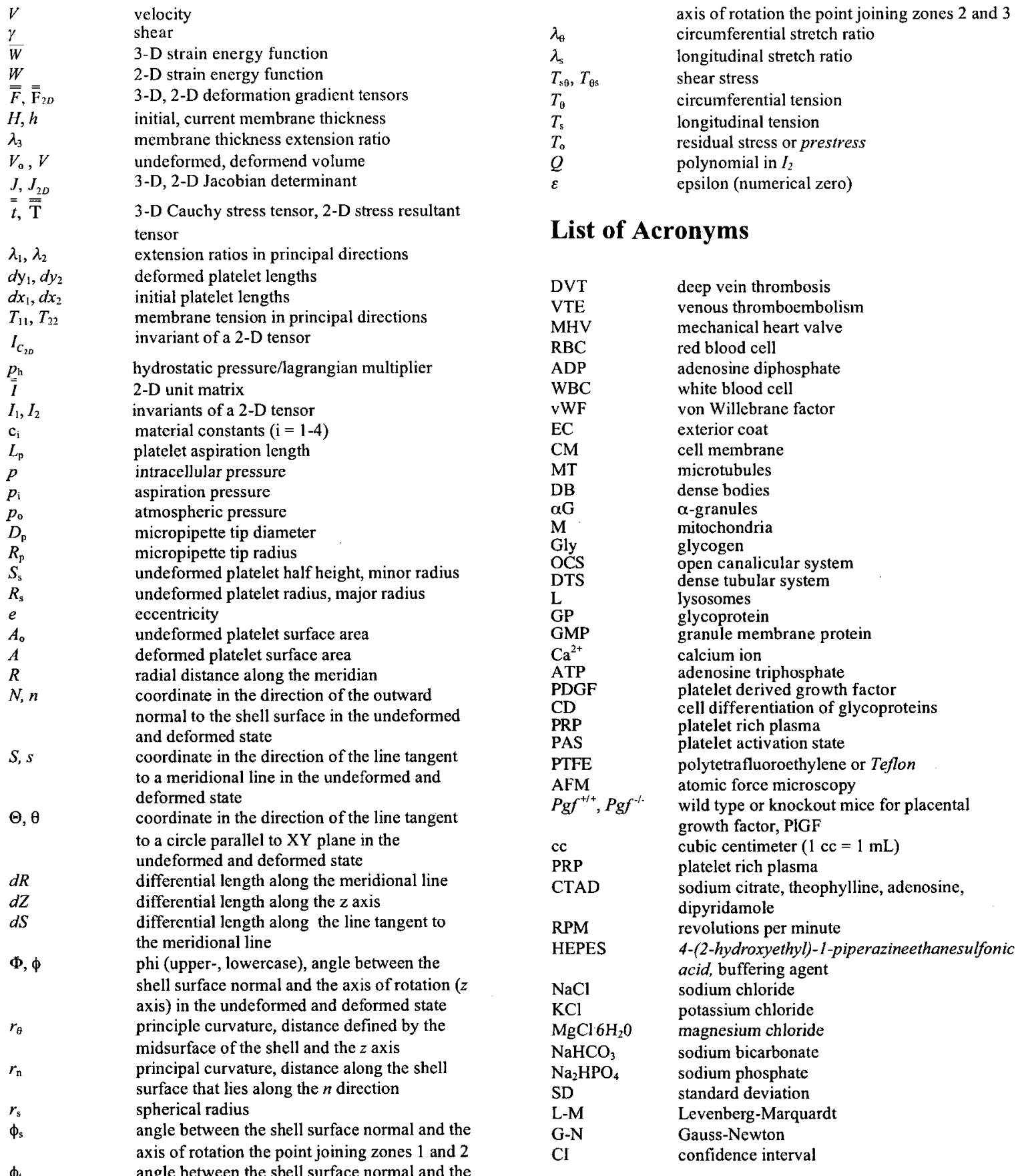




\begin{abstract}
To investigate the mechanical behavior of platelets in response to an applied force, the deformation capacity of the membrane, as quantified by membrane progression into a borosilicate glass micropipette of defined internal diameter, is probed using a controlled range of negative pressure $\left(0-7 \mathrm{~cm} \mathrm{H}_{2} \mathrm{O}\right)$. In real-time, murine platelet membranes fail to demonstrate viscoelastic effects, but maintain an average linear deformation behavior $(n=11$ platelets). Along with published deformation data using human platelets, a novel constitutive model generates a set of optimized material constants that accurately predict platelet membrane behavior. Using MATLAB (Version 7.4), the model is able to preserve platelet deformation at constant volume by implementing the Levenberg-Marquardt method, and ultimately generates an experimentally validated simulation program of micropipette aspiration. Finally, as a complement to these products, the tension and stretch responses to a specific magnitude of negative pressure are resolved.
\end{abstract}




\section{Acknowledgements}

I sincerely appreciate the guidance and support received from my supervisor, Dr. Michel Labrosse, to whom I owe a great deal of thanks. I would also like to extend my gratitude to Dr. Robert Monette, Dr. Geoff Mealing, Tanya Comas and Katharine Schaefer of the National Research Council's Institute for Biological Science and Dr. Antonio Giulivi of the Ottawa General Hospital for their warmth and expertise, as well as their equipment contributions. I am also obliged to the Department of Mechanical Engineering, the Faculty of Graduate Studies, and my supervisor for the generous financial support that has enabled my research.

I dedicate my thesis to my parents and Shane, who have nurtured my potential with unconditional love and support throughout this experience, which has proven invaluable in each new challenge. I am forever grateful. 


\section{Chapter 1}

\section{Introduction}

\subsection{Introduction to the Platelet}

An individual's quality of life hinges on a complex systemic balance that can easily shift to a state of illness or disease. Notably, circulatory system imbalance causing thrombosis presents a real risk in modern society, contributing significantly to morbidity and mortality rates and leading $50 \%$ of the annual non-accidental deaths in the United States (1). Thrombosis is a salient factor in peripheral, coronary, cerebrovascular and nonspecific vascular afflictions induced by hemostatic imbalance or exogenous devices. At the periphery, deep venous thrombosis or DVT can potentially induce venous gangrene and/or pulmonary embolism (2) at an annual incidence of $1.6-1.8$ per 1000 people, and is most commonly asymptomatic in nature ( $96 \%)$ (3). In the same realm of severity and manifestation, the incidence rates of venous thromboembolism (VTE) parallels that of stroke victims, and is more fatal than acute myocardial infarction (9). In contrast, thrombosis in cardiovascular disease (i.e. valve malfunction) and exogenous prosthetics (i.e. MHVs, stents) is predominantly symptomatic, potentially resulting in stroke, myocardial infarction and cardiac death (4). Clearly, the development of each pathophysiological condition is unique in terms of mechanism and systemic location, but perhaps share a common microenvironment that encourages these thrombotic events. Of interest to the current work, one must therefore consider the role of mechanical stimuli in the differential regulation of thrombosis, of which abnormal blood flow conditions, like stagnation or exposure to high shear stress, are induced by arterial stenosis or mechanical heart valves (MHVs) $(5,6,7)$.

In clinical settings, the incidence and progression of each hemostatic malady is inconsistent in terms of therapeutic approach, and are limited by anticoagulant agents and antiplatelet therapies that vary in efficacy and patient safety (8). To address these inadequacies, one might begin to improve patient-specific care by further investigating the mechanical environment of each condition to ascertain thrombogenic risk. Specifically, to reduce the development of these fatal conditions, which may be asymptomatic, one could identify venous regions with high shear stress (i.e. arterial stenosis) and internalized 
prosthetic device designs (i.e. caged-ball, tilting-disk, bileaflet MHVs) (4) for their propensity to induce platelet lysis and ultimate thrombosis. Then, on a fundamental level of investigation, by modeling platelet membrane responses to an applied pressure, one might begin to understand and predict platelet mechanical behavior in circulation and in prosthetic devices. In doing so, a novel or improved therapy that begins to focus on prevention of thrombus development rather than treatment may evolve, which is an approach predominantly lacking in clinical settings (9). These efforts could refine treatments and improve disease management by introducing alternative interventions (i.e. angioplasty, vascular stenting) or by modifying the intensity/duration of pharmacological treatment. Ultimately, the relevance of data collected at all levels of analysis will be considered in the context of improving basic knowledge of thrombosis and clinical practice.

\subsection{Thesis Objectives}

To minimize the scarcity of research detailing the mechanical properties of the platelet membrane, the current work aspires to introduce a novel constitutive model with the capacity to predict membrane responses to an applied load by ultimately defining membrane material constants. Towards this end, one must first propose a constitutive equation that accurately describes membrane mechanical responses to micropipette aspiration, accommodating deformation responses from human and murine platelet samples. This behavior must then be integrated into a comprehensive mathematical model that accurately simulates micropipette aspiration of a platelet. The theoretical foundations of the model must also be validated, employing complementary data sets from the published works of Haga et al. (11) and data collected presently. The presence of mechanotransduction pathways and the limits of platelet membrane strength must also be investigated for their future relevance in clinical applications. Ultimately, the sum achievements of this work will amount to a more comprehensive mechanical description of the platelet membrane. 


\subsection{Thesis Contributions}

\subsubsection{Major Contributions}

As dictated by the generation of accurate membrane material constants, the accumulated efforts of this thesis generate a theoretically validated mathematical model that accurately predicts platelet membrane behavior to a specific mechanical stimulus. These membrane responses are exemplified in theoretical simulations of micropipette aspiration and graphical depictions of membrane stretch and tension.

\subsubsection{Minor Contributions}

Limited evidence of platelet lysis following the application of a specific magnitude of negative pressure has enabled a preliminary definition of membrane strength using the mathematical model.

\subsection{Organization of Thesis Dissertation}

To engage potential readers, the thesis begins with an extensive literature review (Chapter 2) that details the generalized structure and function of the human platelet, and relates this description to the murine platelet species using validated homologies. The significance of this comparison is made evident in later experimental applications that verify theoretical uncertainties in the mathematical model and tests the feasibility of micropipette aspiration using modern technologies. The literature review then details the macroscopic mechanical catalysts of platelet activation and aggregation and narrows the focus to a facet of platelet research that is largely unsupported in terms of published works. Specifically, the microscopic mechanical properties of the platelet, considered either on a global or local scale, are mainly absent due to the relative difficulty of in vitro cell manipulation. However, advances in single cell manipulation techniques that accommodate the high sensitivity and small size of the platelets are detailed. Of specific interest to the current work, micropipette aspiration proves to be an ideal candidate, as it is commonly used for small-scale cellular applications (i.e. erythrocytes, platelets) and contributes a simplistic set of deformation data that facilitates a comprehensive mechanical description of the platelet membrane. The minor complexities of the data set are subsequently exploited in a constitutive model that follows a 
detailed set of principles and methods that are similarly applied in alternative constitutive membrane models.

Next, the dissertation details the mechanisms of blood sample extraction, platelet isolation and micropipette aspiration that generate an acceptable deformation data set by preserving the inactive state of the murine platelet (Chapter 3). Along with published deformation data using human platelets, a comprehensive description of platelet geometries in the undeformed and deformed state marks the beginning development of a mathematical model (Chapter 4). Constitutive descriptions of the general material behavior of the platelet membrane are also defined. Subsequently, the model is implemented in MATLAB and tested for its capacity to generate optimized material constants and novel mechanical properties for the platelet membrane. These results are then compiled and discussed based on compatibility to experimental evidence and their ability to predict membrane responses to an applied load (Chapter 5). Finally, the limitations of the current work are discussed, with recommendations to ameliorate the model and improve the impact of results in future applications. 


\section{Chapter 2}

\section{Background and Literature Review}

The following chapter begins with a detailed description of the functional and structural complexities of the human platelet, emphasizing the importance of a biochemically controlled experimental setup that limits largescale platelet activation caused by positive feedback mechanisms. As detailed below, the conservation of these properties justifies the implementation of a murine platelet model for subsequent inferences to human platelet mechanical behavior. This chapter also omits descriptions of thrombotic events induced by damaged vasculature, in an attempt to focus on the role of mechanotransduction pathways at the macro- and microscale. Notably, the growing field of single-cell mechanical manipulations is detailed, as these techniques generate information that proves invaluable to constitutive modeling of cellular behavior. The general principles and methods of constitutive modeling, along with a relevant erythrocyte membrane model, establish method feasibility in characterizing platelet membrane mechanical properties.

\subsection{Human Platelet Background}

With specific interest to the process of coagulation, whole blood possesses a functional versatility fostered by its variability in aqueous and cellular phases. Blood plasma is predominantly water $(\sim 90 \%)$, but possesses over 100 various dissolved proteins and ions that contribute significantly to plural intermediate coagulation reactions. The cellular components of blood ( $46 \%$ of the volume in human blood) consist primarily of red blood cells (RBCs) (or erythrocytes) ( $\sim 98 \%$ in cell number), which may stimulate blood clot formation upon release of an intracellular aggregation agonist (i.e. ADP) (12). The remaining $2 \%$ of the cellular phase consists primarily of white blood cells (WBCs) (or leukocytes) and platelets (5, 13), which play major roles in immune and thrombotic processes, respectively. The total volume of platelets represents less than $0.2 \%$ of the cellular components.

Synthesized in bone marrow, each platelet reaches a volume of 6 - 10 femtoliters (14). In its dormant state, the inactive discoid platelet has a $2-5 \mu \mathrm{m}$ diameter (14). Cellular integrity is maintained by the cytoskeletal components of the platelet, which act to withstand 
the forces generated by blood flow over the endothelium (14). The structural elements of this system include a spectrin framework (15) that interfaces with the cytoplasmic side of the plasma membrane, a microtubule coil that lies along the circumference of the cell (11) and finally a stable network of cross-linking actin-filaments that spans the entire cytoplasm (Figure 2.1).

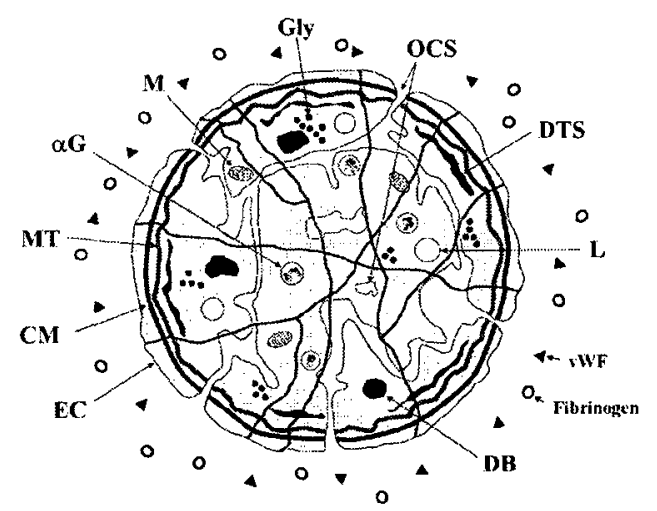

Figure 2.1: Diagrammatic, cross-sectional representation of the normal human platelet structure with its actin cytoskeletal filaments, and von Willebrand factor (vWF) and fibrinogen from circulating blood plasma. Shown are the exterior coat (EC), cell membrane (CM), microtubules (MT), dense bodies (DB), $\alpha$-granules ( $\alpha \mathrm{G}$ ),

mitochrondria (M), glycogen (Gly), open canalicular system (OCS), dense tubule system (DTS) and lysosomes (L).

The outermost layer of the platelet, known as the glycocalyx or external coat, is a surface coat exposed to the aqueous plasma medium that consists of glycoproteins (GP) and is $15-20 \mathrm{~nm}$ thick $(11,16)$, a magnitude which makes this exterior coat much thicker than in other blood cells (14). Inferior to the glycocalyx is the platelet cellular membrane with a thickness of $10 \mathrm{~nm}$ (11), with a phospholipid component (notably phosphatidylserine) that promotes the progression of the coagulation cascade by providing a catalytic surface for Factor-X and prothrombin activation (17). Platelets also possess an open canalicular system (OCS) whose channels connect to the interior of the platelet and serve as a transportation route for constituents released upon platelet activation (18). Prominent cytoplasmic organelles include mitochondria, alpha $(\alpha)$ granules, electron dense bodies, peroxisomes and lysosomes $(14,19)$. In view of the variable functions of organelle-enclosed substances (Table 2.1), the significant ramifications of cell rupture or biochemical release in generating thrombotic events are clear (Section 2.1.1). 
Table 2.1: Various functions of organelle-enclosed molecules (a to d). [Adapted from (16)]. (a)

\begin{tabular}{|c|c|}
\hline \multicolumn{2}{|c|}{ Alpha granules } \\
\hline Molecule & Function \\
\hline Fibrinogen (platelets, plasma) & platelet aggregation (20) \\
\hline Factor $\mathrm{V}$ & $\begin{array}{c}\text { cofactor in coagulation ( } 22), \text { not enzymatically } \\
\text { active }\end{array}$ \\
\hline Platelet-derived growth factor (PDGF) & encourages angiogenesis (14) \\
\hline Platelet factor 4 & $\begin{array}{l}\text { heparin antagonist (16), angiogenesis inhibitor } \\
\text { (23) }\end{array}$ \\
\hline Beta thromboglobulin (platelet specific) & $\begin{array}{l}\text { clotting factor, stimulates neutrophils } \\
\text { (chemotaxis, degranulation, adhesion) (24) }\end{array}$ \\
\hline Albumin & $\begin{array}{c}\text { maintains blood volume (25); impairs platelet } \\
\text { aggregation (26) }\end{array}$ \\
\hline Fibronectin & platelet adhesion (16) \\
\hline Thrombospondin & angiogenesis inhibitor (14); platelet adhesion (16) \\
\hline $\begin{array}{l}\text { von Willebrand factor ( } \mathrm{vWF} \text { ) (platelets, endothelial } \\
\text { cells) }\end{array}$ & $\begin{array}{l}\text { platelet-platelet adhesion (16) binds to thrombin } \\
\text { and ADP (27) }\end{array}$ \\
\hline
\end{tabular}

(b)

\begin{tabular}{cc}
\hline & Electron Dense Granules \\
\hline Molecule & Function \\
\hline Calcium $\left(\mathrm{Ca}^{2+}\right)$ & platelet aggregation (16) \\
serotonin & platelet aggregation (16) \\
adenosine diphosphate (ADP) & platelet aggregation (16) \\
\hline
\end{tabular}

(c)

\begin{tabular}{cc} 
& Lysosome \\
\hline Molecule & Function \\
\hline Hydrolytic enzymes & unknown role in hemostasis (14) \\
\hline
\end{tabular}

(d)

\begin{tabular}{ccc}
\hline & Peroxisomes & \\
\hline Molecule & Function \\
\hline Catalase & mediates platelet activation (21) \\
\hline
\end{tabular}

Evidently, the platelet is one essential blood component regulating the haemostatic process because of its adhesive, aggregative and clotting abilities that govern the coagulation cascade. For the same reasons, the platelet is also significant in pathological thrombosis. 
Typically platelets are highly sensitive to mechanical and chemical stimuli, promoting a series of large morphological and chemical changes representative of the transition into the platelets activated state. Catalysts for platelet activation include injury to vasculature, contact with exogenous foreign surfaces, imbalance of pro- and anti-coagulants in the intact endothelium ('endothelial dysfunction') (10), or abnormal blood flow conditions like stagnation or exposure to high shear stress induced by arterial stenosis or mechanical heart valves $(6,7,5)$ as discussed in Section 2.2 .

\subsubsection{Platelet Activation}

The process of platelet activation is marked by a centralization of platelet organelles, surface glycoprotein conformational changes, and the extension of long spiky processes called pseudopods or filopodia (5). The structural changes in glycoprotein receptors lining the glycocalyx facilitate the adhesion and thus aggregation of platelet populations during hemostatic events (28). These receptors mediate interactions with various adhesive agents, aggregating agents, inhibitors and procoagulant factors (16). Specific to platelets, the selectins (granule membrane protein GMP 140 or P-selectin), the integrins (GP I to GP V), the immunoglobulins (GP VI) and additional receptors with affinity for ADP, collagen, thrombin and epinephrine have significant roles in thrombogenesis $(14,28)$. In addition to the specific roles of GP Ia-IIa (integrin $\alpha 2 \beta 1$ ), GP Ib-IX-V and GP IIb-IIIa (integrin $\alpha I I b \beta 3$ ) in facilitating the adhesion of activated platelets to collagen, vWF and fibrin respectively (14, 24), the cell surface receptor P-selectin mediates the adherence of neutrophils and monocytes during the inflammatory response $(16,20,30)$. Contrary to the glycoproteins that perpetually reside on the glycocalyx surface, P-selectin is solely expressed on the platelet membrane surface when platelets are activated and thus degranulate (31). The organelle centralization is a characteristic feature of the degranulation event, in which respective platelet aggregation and autocrine agonists such as ADP and serotonin are released from the dense bodies, while $\alpha$ - and lysosomal granules release other components to further facilitate the activation of adjacent platelets (14). Degranulation is a result of $\alpha$-granule and dense body fusion to the open canalicular system (OCS) of channels that interface with the outer membrane of the platelet $(11,18)$. The fusion of platelet organelles facilitates the translocation of the intracellular metabolites to the extracellular plasma medium, leading to exponential 
activation of neighboring platelets. These chemicals stimulate the increased expression of cell membrane adhesion receptors such as GP IIb-IIIa, which also encourages the recruitment of platelets to the site of injury (29). During this time, a transient chemical change occurs with a notable change in cytoplasmic calcium $\left(\mathrm{Ca}^{2+}\right)$, whose increased intracellular concentration induces contraction of the platelet via the activation of the actino-myosin motor complexes responsible for platelet cell shape (5). The activated platelet undergoes a morphological change in which it conforms to a roughly spherical shape and loses its previously discoid appearance. This new shape, in conjunction with the extension of filopodia, facilitates the mass aggregation of platelets that forms the base of the final clot by increasing the probability that adjacent platelets will collide due to the relative increase in surface area of each platelet (5).

As the scope of the current work is fundamentally focused on the mechanical behavior of the platelet membrane, one must appreciate the generality of this description of platelet structure and function. For further details about the biochemical complexities of platelet function and hemostasis, Michelson's 'Platelets' (14) and the review article by Stassen et al. (29) are helpful references.

\subsubsection{Platelet Aggregation}

During aggregation, platelet-platelet interaction is reinforced by the contributions of vWF, a large plasma protein that also bridges the gap between the damaged tissue and platelets by means of its shear-induced connection to the platelet membrane glycoprotein receptors (GP Ibo) $(26,29,32)$. Collagen and vWF engage the GP VI and GP Ib-IX-V complexes to induce events such as platelet shape change, aggregation and secretion (14), with notable activation of the GP IIb-IIIa receptor to increase its adhesive properties via shear stress-induced vWF binding to the GP Ib-IX-V complex $(26,33)$. These agonists are also important initiators in the prominent conformational change of the platelet membrane glycoprotein GP IIb-IIIa receptor, which exposes a fibrin receptor and a site for binding of soluble ligands (namely fibrinogen) and thus becomes a catalytic location for platelet-platelet interaction $(34,35)$. The resultant platelet aggregation is encouraged by cross-links between adjacent platelets by the mutual binding of multivalent vWF and divalent fibrinogen (36).

Typically, platelets only adhere to themselves or to the endothelial wall linings when 
damage is present and collagen is exposed (14), or adhere to the surfaces of man-made biomaterials (7). However, the rate and extent of platelet adhesion, deposition and thrombus formation are affected by the blood flow conditions as well (37), and on a less significant scale, the hemoglobin and ADP released by damaged erythrocytes also encourage platelet activation. Upon initial aggregation, platelets are activated by thrombin and their membrane phospholipids release arachidonic acid then converted to thromboxane $A_{2}(14,29)$, a shortlived prostaglandin derivative. Thromboxane $\mathrm{A}_{2}$ then supports additional aggregation of adjacent platelets to the clot formation site (14). After the arachidonic acid release, there is also stimulation to release platelet metabolites such as ADP, which reinforce the continued local aggregation of more platelets, while also exposing the platelet $\alpha$-granule contents (such as fibrinogen, vWF and platelet factor 4) to the extracellular fluid (29). Ultimately, the formation of the platelet clot provides a loosely-knit basic foundation that the integration of formed fibrin will reinforce by providing a reliable structural support in the coagulation cascade (29).

\subsubsection{Comparison of Structure and Function between Human and Mouse Platelets}

As evidenced in a number of studies, the exploitation of mouse blood samples proves convenient for in vivo and in vitro studies of the biological properties of platelets (38). Any direct comparisons between the respective biological functions of each species must be made with caution, however, as the common integration of transgenic and knockout mice in studies may alter their natural biological processes (38). In fact, the manipulation and investigation of mouse models with altered genetic properties has contributed significantly to the understanding of several biological and pathophysiological mechanisms in humans $(39,40)$. Nevertheless, to make justifiable inferences about human biology through murine model investigations it is imperative that a more comprehensive understanding of mice in healthy or diseased states be achieved. As such, the inherent and possibly significant morphological differences between these two species must be explored, with specific interest to possible roles in differential platelet function. 


\section{Structural Comparison:}

From a morphological perspective, both light microscopy and electron microscopy analysis have identified many structural similarities and differences between human and mouse platelets (41). Of significance, murine platelets are prevalent in circulation at greater numbers, and support the smaller mammal with a comparably diminutive size (Table 2.2) to the human alternative (38).

Table 2.2: Comparison of platelet parameters in the human and murine species $(42,38,14,46)$.

\begin{tabular}{|c|c|c|}
\hline & Human & Mouse \\
\hline platelet counts $\left(\times 10^{9} / \mathrm{L}\right.$ in blood $)$ & $150-400$ & $1000-1500$ \\
\hline platelet diameter $(\mu \mathrm{m})$ & $2-5$ & 0.5 \\
\hline mean platelet volume $(\mathrm{fl})$ & $6-10$ & $3-4.7$ \\
\hline
\end{tabular}

In terms of composition, the intracellular components of murine platelets are also quite similar to those of human platelets. Impacting the current research due to their procoagulant behavior, the contents of dense granules (serotonin, ATP, ADP) (43) and $\alpha$ granules (fibrinogen, PDGF, vWF, platelet factor 4) (16) were identified. Of functional significance, conservation of prominent platelet membrane glycoproteins between species emphasizes the importance of these receptors in mediating proper platelet function, as evidenced in immunoelctron microscopy experiments (38).

Although mouse glycoproteins (GP) have yet to be extensively investigated, a number of these receptor complexes (GPIIb/IIIa) are homologically similar to the human alternative (42). The significance of such a discovery was confirmed after the breeding of mice lacking this amino acid sequence (i.e. GPIIIa-knockout mice), as scientists discovered increased mortality of these genetically altered mice, possibly due to hemorrhage (42). Further, platelet analysis using these GPIIIa-knockout mice exhibits almost identical phenotypes to that of the bleeding disorder human Glanzmann thrombasthenia, suggesting some pathophysiological parallels to human hemostasis (44). To date, comparisons between murine and human proteins with significant roles in hemostasis demonstrate high amino acid homology, thereby conserving to a certain degree the structure and functions of essential coagulation proteins (Table 2.3) (44). Given these relationships, however, it is still important to recognize that some functional and structural differences exist and require consideration. 
Table 2.3: Amino acid homologies of cloned murine platelet coagulation proteins to human alternatives. [Adapted from (42)].

\begin{tabular}{|c|c|c|c|c|c|c|c|}
\hline Protein & $\begin{array}{c}\beta 3-\text { integrin } \\
\text { (GPIIb/IIIa) }\end{array}$ & GPIb $\alpha$ & GPIb $\beta$ & GPV & GPIX & $\begin{array}{c}\text { Factor } \\
\text { VII }\end{array}$ & $\begin{array}{c}\text { Factor } \\
\text { VIII }\end{array}$ \\
\hline Homology & $92 \%$ & $75 \%$ & $88 \%$ & $70 \%$ & $71 \%$ & $70 \%$ & $74 \%$ \\
\hline
\end{tabular}

As mentioned, immunoelectron microscopy is a helpful technique used to identify morphological differences and map protein homologies between species, such as confirming $\alpha$-granule protein conservation, but has also revealed the disparities between ultrastructural localization of proteins (38). Murine platelets have increased heterogeneity of granule shape and size to that of human platelets, and the organization of glycoproteins within murine platelet $\alpha$-granules is of a highly compartmentalized orientation (38). Immunoelectron microscopy demonstrates species similarities between $\alpha$-granules, with asymmetrical dispersion of von Willebrand factor (vWF), and the prevalence of GPIIb/IIIa and P-selectin (CD62P) along the granule membrane (38). Additionally, dense granules between species are of similar appearance, but are less prevalent in murine platelets, even when platelet surface area is controlled (38).

The use of immunolabeling for the comparison of granule and plasma membrane proteins between mice and humans also revealed differential expression patterns and morphologies for several proteins, but confirmed the usefulness of mouse models for the study of human platelet biology and function $(45,38,46)$.

\section{Functional Comparison:}

Logically, establishing a well-founded connection between the ultrastructure and consequent functional attributes of both murine and human species is a prerequisite when attempting to use murine models for the study of human pathology. Specifically then, any morphological or functional variability between murine and human platelets must be defined, which may have significant repercussions when attempting to derive important conclusions regarding their respective roles in pathological thrombosis. Murine platelets are recognized to be genetically similar to their human counterparts, and remain functionally similar to human platelets regardless of their differing size, number and structure (46). Along with the mouse adaptability to genetic manipulation, the functional similarities between these two species have proven advantageous in improving knowledge of platelet contributions to hemostasis 
and pathological thrombosis (46). In terms of coagulation, this highly regulated system in mice is recognized to be quite similar to that of humans, with specific reference to the structural conservation of coagulation factors (47). Specifically, homology between amino acid sequences of coagulation factors for human and mouse models demonstrates similarities in domain structures (Table 2.3), suggesting that there are indeed fundamental coagulation processes maintained in both species (42). Further, the structural modifications during murine platelet activation parallel those of human platelets, as shape change in activated mouse platelets is dependent on the actin assembly in response to most agonists (42).

In conjunction with this actin reorganization, murine P-selectin similarly translocates from its resting state on the $\alpha$-granule membrane to the site of organelle content release, the plasma membrane (42). This adhesion molecule also bears similar functional roles to human platelets, made evident in $\mathrm{P}$-selectin deficient mice that demonstrate symptoms of prolonged bleeding and increased bleeding at inflammatory lesion sites (39).

Evidently, there are many distinguishing differences between murine and human platelets (size, number, histological distributions) that are worthy of attention (38). Regardless of these disparities, similarities in intracellular distribution (organelle and glycoprotein subcellular distribution) and function do indeed exist between these species, thereby justifying the study of mice models to investigate various human platelet biological properties (38).

\subsection{Macroscopic Platelet Biomechanics in the Literature}

The following chapter presents the significant effects of variable flow environments in circulation that contribute to platelet activation and aggregation. Evidently, the thrombogenic impact brought about by these systems of irregular blood flow emphasizes the role of mechanical contributions to circulatory integrity.

\subsubsection{Shear-Induced Platelet Activation}

The most extensive facet of platelet activation research thus far focuses on the physical and biochemical repercussions of shear stress generated by flowing blood (26). The motivation of such research is to understand thrombosis as it occurs in injured or diseased blood vessels. 
Shear stress is defined as the force per unit area between fluid layers (or laminae) in flowing blood, which contains an inifinite number of interfacing laminae having some frictional interaction with its neighbors (26). Assuming that the shear stress between laminae is proportional to the shear rate, defined as the change in velocity between two laminae (definition of a Newtonian fluid), then the blood flow through a uniform cylinder takes on a parabolic velocity profile with a maximum shear stress value at the periphery (26) (Figure 2.2):



Figure 2.2: Blood flow in a cylindrical chamber of radius ' $R$ ' generates a parabolic flow velocity profile. This results in maximal velocity $(v)$ and minimal shear $(\gamma)$ at the center of the blood flow stream, and minimal velocity and maximal shear at the vessel wall. It is the difference in velocity between laminae of flowing blood that generates shear forces.

This simplistic model of blood flow through a cylinder with uniform cross-sectional area fails to accurately model blood flow conditions in vivo. Platelets having roughly the shape of an oblate spheroid (or discoid), they would theoretically be subjected to alternating normal and tangential fluid stresses during each rotation induced by the shear stress field within the blood flow. However, at normal hematocrits (the RBC concentration per unit volume of whole blood), particle crowding is such that free rotation of platelets and RBCs is all but inhibited. The continuous collisions between the predominant RBCs induce mixing of the far less numerous platelets and leukocytes, and increase the rate of collisions between the platelets and the vessel wall $(26,12)$. Pulsatile flow conditions with dynamic constrictions dominate in health as well as in diseased states (26).

Nevertheless, preliminary testing of thrombosis was predominantly performed in static and stirred mediums (12), which proved insufficient in characterizing thrombotic disease of the coronary and cerebrovascular dynamic circulation (48) since the magnitude of 
shear stress was low and varied in a complex and uncharacterized way (26). Improvements to mechanical models using circular flow tubes designed to replicate high shear stress in relatively short periods of time $(25-1600 \mathrm{~ms})$ demonstrated that platelet stimulation is a nonlinear phenomenon which changes as a function of shear rate and time, but failed to apply a uniform exposure time and shear stress to all fluid elements (49). It was concluded from circulating flow loop models, that shear stress effect on platelet activation, like chemical agonists, is dependent on 'dose' as well as duration (50). Sutera et al. (51) also established that pulsed exposure to shear stress yielded increased platelet aggregation compared to continuous exposure. A study by Boreda et al. (52) elaborated further and showed that the magnitude of shear stress is more significant than that of exposure time. Mechanical models were then refined based on relevance to the arterial circuit, which is recognized as a high flow environment (53). This was done because modeled mechanisms inducing platelet activation and aggregation must reflect physiologically relevant flow conditions as well vascular geometry $(54,6)$. Specifically, mechanical models that simulate injured or stenotic blood vessels were implemented to replicate vascular responses to hemodynamic forces in vivo using controlled mechanical forces in vitro with a blood suspension assumed to behave as a Newtonian fluid (53). Jesty et al. (50) found that platelet rich plasma (PRP) flow through a stenotic loop produced a 5 -fold increase in platelet activation compared to that of a nonstenotic loop (Figure 2.3), indicating a need to characterize platelet activation at multiple shear stress magnitudes (32). Wootton et al. (55) found the region of highest shear to be located within the stenosis throat, which positively correlates to the region with greatest thrombus formation and thus platelet activation (56). 


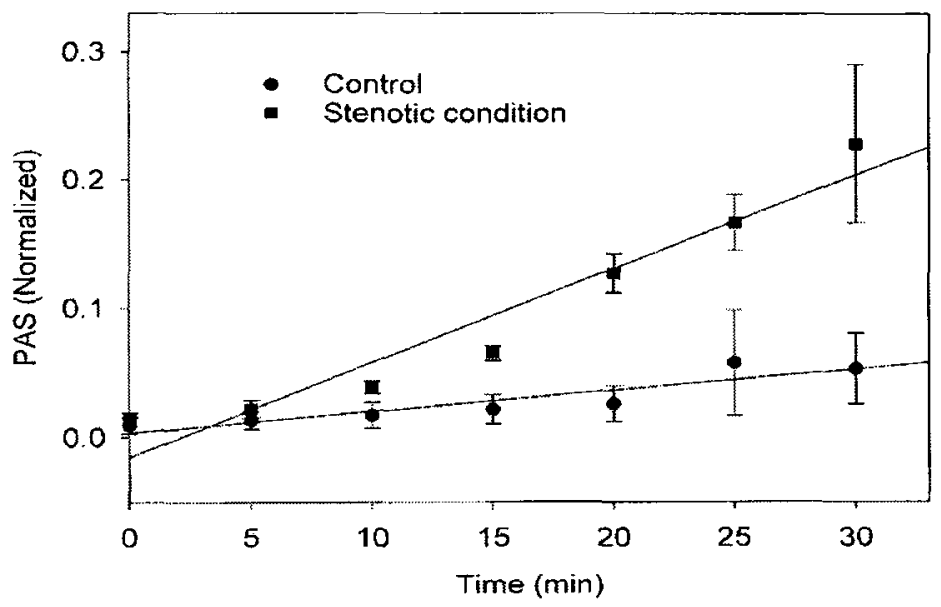

Figure 2.3: The effect of a stenotic region on the activation of platelets in a circulation loop. Gel-filtered platelets were circulated in a flow loop in a platelet buffer at a flow rate of $6.25 \mathrm{ml} / \mathrm{min}$. Samples were removed immediately after injection into the loop (zero time), and then at 5-min intervals, for assay of platelet activation state (PAS) $(\bullet$ ). In a parallel experiment a $1 \mathrm{~m}$ section of $0.97 \mathrm{~mm}$ PTFE tubing was inserted in the circulation loop to provide a region of high shear rate $(\mathbf{\square})(50)$.

It is important to note that shear stress exposure is different for platelets that circulate through stenotic arteries $(26,55)$, due to different transient exposure times and shear stresses (12). By contrast, platelets that accumulate in a growing thrombus are continuously exposed to shear until the ultimate occlusion of the vessel. Experimental evidence supports that the threshold for platelet activation is dependent on both platelet-solid surface interaction and duration of platelet exposure to shear stress. Novel mechanical models then emerged to directly attribute platelet activation to fluid shear stress using rotational viscometers coated with silicone (or another nonthrombogenic material) that can differentially apply pulsatile shear stress (52) or allow real-time optical measurements of platelet aggregation (57), with both designs generating a constant and uniform shear stress. These models were used to explore the variability of shear-induced platelet activation and aggregation, which resolved a number of suppositions that shear stress did not induce platelet activation, but rather caused platelet lysis (58) exposing a multitude of platelet activation agonists into circulating plasma. This supposition was repeatedly discredited by several studies, namely those using rotational viscometer systems $(56,12)$ that found that platelet activation depends on the presence of vWF and functional platelet glycoprotein complexes GP Ib-IX-V and GP IIb-IIIa in conjunction with moderate shear stress magnitudes $(26,59)$. This connection between rheologic and vascular factors is supported by the fact that mixing vWF with platelets in a 
static or stirred suspension will fail to generate a platelet response (26). Although the implementation of viscometers generating well-controlled shear fields has yielded a multitude of information regarding platelet activation, these conditions are obviously less applicable to vascular flow conditions or flow through prosthetic devices (50).

\subsubsection{Mechanical Heart Valves and Thrombosis}

Although the use of mechanical heart valves (MHVs) as a long-term therapy is far superior to any drug treatment offered to date, mechanical valves are at significant risk of causing platelet rupture, hemolysis and thromboembolisms, all of which prove unfavorable to longterm use (60). Note that such deleterious effects are mostly not observed with bioprosthetic heart valves, which have structural longevity problems of their own. Given that the materials used for manufacturing MHVs are selected for their low intrinsic thrombogenicity, it follows that the function of MHVs is thrombogenic. Several scenarios can be imagined, including a most extreme one in which crushing of platelets occurs between fixed and moving rigid parts of the MHVs. Release of the platelet content in the bloodstream due to cell lysis will indeed trigger a massive thrombotic reaction (58). MHVs are also known to induce cavitation, which is the formation and collapse of vapor-filled bubbles (61). Cavitation is an energetic, albeit very localized, phenomenon that has been observed to promote erosion of the surface of MHVs. Therefore, it could possibly be involved in pro-thrombotic effects, by still unknown mechanisms (61).

Despite these potential mechanical effects of MHVs on platelets, it is the shear stress generated by flowing blood around MHVs that has received the most attention from researchers. As a control, biomolecule-induced platelet-platelet aggregation and adhesiondependent activation were prevented by exclusion of the plasma protein agonists that induce these processes, most notably fibrinogen, vWF and erythrocytes (7). In vitro studies then established the procoagulant properties of platelets in flow conditions (mildly restricted, severely restricted, unrestricted) mimicking those produced by MHVs, using a left ventricle assist device with two Björk-Shiley mono-leaflet valves mounted in opposition that generated areas of high shear stress, turbulent fluctuations, and regions of recirculation and stasis (7). An eight-fold increase in platelet activation for the severely restricted valve was observed when compared to that of the unrestricted valve. This established that restriction is 
one primary source of increased thrombogenicity in MHVs (7), which is marked by increased platelet activation, reduced platelet half-life, and increased thromboxane $\mathrm{A}_{2}$ synthesis and release (62). As with shear-induced platelet activation in stenotic vessel models, high shear stress exposure to only a small platelet fraction is necessary to induce the aforementioned activation resultants, as most pass far from the wall and leaflet and experience low shear stress (63). Experiments in MHVs also proved that platelets are increasingly aggravated over time and are susceptible to activation during both systolic and diastolic phases. Restricted valves in systole produced a recirculation zone that generates local turbulent stresses of the order of several thousand dyne $/ \mathrm{cm}^{2}$ : shear stress magnitudes at which platelet activation is a certainty (63). Diastole causes additional platelet complications, as restriction prevents valve closure, producing retrograde flow resulting in strong regurgitant jets that similarly induce strong shear and turbulent stresses $(37,7)$.

\subsection{Cellular and Subcellular Platelet Biomechanics in the Literature}

The following chapter addresses the relative absence of single-cell and subcellular investigations of platelet biomechanics, and emphasizes the importance of platelet research at a more fundamental level. To date, platelet research probing potential mechanical catalysts of platelet activation and aggregation predominantly considers these events at the whole blood level, and fails to adequately consider the catalytic potential of single cells. As introduced with limited detail below, further research detailing microscopic, mechanicallybased platelet responses will minimize the information gap.

As detailed in Section 2.2, extensive research at the macroscopic level has characterized the biomechanical significance of platelet activation in variable flow conditions with specific emphasis on pathophysiological thrombosis induced by stenotic flow systems and MHVs. To refine the understanding of this biomechanical relationship it is imperative that experimental data be obtained for the platelet mechanical properties at the single-cell level. Indeed, if it is true that platelets are sensitive to shear stress, the precise mechanism has yet to be defined. One such explanation introduces the possibility of shear-stress induced deformations/conformational changes in platelet membrane receptors or the cytoskeleton, which may then be converted to a biological response in the cell $(64,60)$. Additional speculations introduce an actual shear-stress receptor on the cell-surface, or question the 
relevance of the platelet discoid shape and membrane structure with regards to facilitating platelet activation. One such study showed that a deficiency in the stable GP Ib-IX-V complex (characteristic of Bernard-Soulier's Syndrome - BSS) produces prolonged bleeding time, thrombocytopenia, severe hemorrhagic manifestations, and notably increased membrane deformability when compared to normal platelets $(65,14)$. The authors of the study postulated the possible loss of the major transmembrane link of GP Ibo with the actinbinding protein (filamin) inside the platelet membrane to the underlying actin cytoskeleton ( $\sim 85 \%$ of linkages to actin) as being responsible for this unusual deformability $(67,14)$. In other words, a specific magnitude of membrane agitation may induce enough stress on the surface to either render GP Iba receptors nonfunctional or mechanically sensing. This potentially creates an opportunity to investigate whether direct membrane disruption induces a mechanochemical transduction pathway ('mechanotransduction') linking the cell membrane to the potentially reactive cell interior (68).

As mechanotransduction is confirmed in other cell studies $(69,119)$, some researchers suggest that structural changes in the actin cytoskeleton occurring in platelet spreading (14) and cell deformation (70) enable the physically connected GP IIb-IIIa receptor to assume a conformational change required for ligand binding $(71,14)$, which represents a type of inside-out signaling. At the opposite end of this spectrum, proteins (namely GP Ib-IX-V) embedded in the platelet surface regulate actin architecture of the cytoskeleton and control shape change (14), which is an intricate interaction that depends on the cytoplasmic dynamics of the actin polymer. Despite these associations, many questions remain regarding the regulation of such an interaction, the properties of the binding sites, and the functional ramifications of this connection on GP Ib $\alpha$-dependent adhesion (14). For instance, it appears that cytoskeletal associations affect the adhesive function of the GP IbIX-V complex (14), but its pathophysiological significance has yet to be confirmed. Overall, these arguments suggest that any in vivo type of direct platelet membrane deformation such as induced by MHVs may induce shape change and aggregation and thus possible thrombosis. Note also that GP IIb-IIIa, besides its adhesive function, generates signals that induce platelet aggregation and spreading (35). This type of phenomenon is referred to as outside-in signaling. To further emphasize the role of a mechanical stimulus on platelet function, aggregation and adhesion to collagen-coated surfaces was shown to depend on GP 
Ib-IX-V-vWF interactions, especially at high shear rates (72). GP Ia-Ila receptors are also necessary for more permanent tethering of platelets to collagen fibres, and the function of these receptors is enhanced with increasing shear rates (73).

\subsection{Methods to Measure Single Platelets' Mechanical Properties}

Mechanical properties herein include any quantifiable relationship between applied forces and observed deformations, either globally (i.e. whole platelet) or locally (i.e. a portion of the membrane). Mechanical properties of interest can be the modulus of elasticity (or Young's modulus), the shear modulus, the loads and/or deformations necessary to achieve platelet lysis or activation, and parameters describing the time-dependent or viscoelastic response of the platelet.

Obvious barriers to the quantification of such properties of the platelet include the relative size of blood components as well as the relatively small range of forces which can be exerted. However, a number of techniques can adequately measure the mechanical properties of single cells $(74,75)$, and have been used to study platelets, as presented below.

\subsubsection{Atomic Force Microscopy}

Atomic force microscopy (AFM) can measure forces with high spatial resolution at both molecular and cellular scales (76) and allows for real-time measurement of mechanical properties of an unfixed platelet membrane as well as surface topography $(77,68)$. The technique relies on a tapered silicon or silicon nitride probe mounted on a cantilever arm. As the probe tip interfaces with a cell, a physical deflection occurs, which can be measured by sensing the change in position of a laser beam reflecting off the cantilever arm. The high resolution of this detection method (displacements $<1 \mathrm{~nm}$ ) is ensured by the precise placement of the laser and detector (68). With this technique, one can collect force-distance curves for local surface locations, ultimately generating a force map image of the surface (77). Recent platelet investigations using this method looked into the local interactions of surface receptor GP IIb-IIIa which has an important role in platelet adhesion, activation, and aggregation $(78,79)$. The high-resolution capabilities of this method enabled direct visualization of single ligand/receptor interactions on platelets in a dynamic and 
physiologically relevant environment, proving the significance of this technique in future identifications of deformation-induced pathological thrombosis at the local level $(76,79)$.

\subsubsection{Optical Trapping ("Optical Tweezers")}

Photons possess momentum as they propagate, thus exerting a small effective force or 'radiation pressure' on a surface when light shines on it (80). A photon beam can then be directed to trap a bead or small particle (80). This trapped bead can be coated with an adhesive glycoprotein (typically fibronectin) which has specific or non-specific affinity for biological membranes, so that the bead will preferentially bind to a given location on the cell

allowing displacement of the membrane by non-invasive means $(80,81)$. Once adhered to the cell surface, the beam is moved laterally, and the motion of the bead can be observed at the microscopic level (82). Exerted force and bead displacement are simultaneously quantified, and can be used to determine the elastic and viscoelastic behavior of the membrane $(81,82)$. As optical tweezers behave like a mechanical spring, this technique is ill-suited for steady-state force measurements that exceed simple motion impedance (80). However, this method has applications relevant to platelets in understanding the variable responsiveness of membrane receptors (ex. GP Ib-IX complex) to specific forces $(80,74,75$, 29). As with AFM, the surface topography of a cell can be obtained. Although the method is beneficial in many biomechanics applications, in that it can generate forces representing the load from few molecules, the method cannot generate cellular-level forces in excess of $\sim 100$ $\mathrm{pN}$ without risking thermal or optical cell damage (80). This proves to be a significant barrier when attempting to model the mechanical repercussions of cell-cell interaction in circulation, as cellular forces are in the $\mathrm{nN}$ range (80). Technological improvements of this simple technique also offer the opportunity to investigate the deformation response of entire cells to large optical forces that limit cellular damage, using an 'optical stretcher' set-up which utilizes two unfocused laser beams in opposition (80). Although this 'optical stretcher' technique offers a similar global evaluation of cellular elastic behavior to that of micropipette aspiration (see Section 2.4.3), it lacks experimental validation with platelet models. 


\subsubsection{Micropipette Aspiration}

Characterization of the mechanical properties of platelets and potential activation can be performed using micropipette aspiration of a single cell (11). This type of mechanical manipulation that investigates cellular and subcellular biomechanical properties dates back to the middle of the twentieth century. At the basic level, it requires the use of a fine glass micropipette whose internal diameter typically ranges between $0.5-10 \mu \mathrm{m}$, and whose tip is controlled by a micromanipulator (68). Under direct microscope observation, a portion of the cell membrane is sucked into the micropipette, which allows for a real-time measurement of the cell's deformation and motion as it responds to quantifiable pressure changes. The mechanical properties (specifically, viscosity, elastic modulus and relaxation time) of erythrocytes and leukocytes have been investigated via aspiration of the entire cell or a cellular fraction under constant negative pressure $(83,84,85)$. Applications of this technique to platelets were initially impeded due to their small size and propensity to become sticky upon contact with glass. These limitations were overcome by limiting the internal diameter of the pipette to below $1 \mu \mathrm{m}$, and applying agents that prevent platelet activation during sample processing (65). With this resolved, micropipette aspiration could potentially enable the characterization of the variable stress and strain information in the membrane, and the determination of the magnitude of stress and/or strain required for platelet activation or ultimate lysis. Although micropipette experiments on platelets have been performed, they concentrated on the general deformation index, defined as the ratio of the magnitude of platelet deformation to imposed pressure without considerations to the time history of platelet tongue deformation $(86,87,88,11)$. In other early studies, platelet viscosity was assumed to be constant and independent of the rate and type of deformation, as allowing the platelet's aspirated cross-section to return to its original geometry in the absence of applied external forces enables a force balance between the viscous resisting forces and the elastic restoring forces $(89,90)$.

Validation of platelet micropipette aspiration is given by a number of studies whose static deformation data is qualitatively similar, with minor differences attributed to evolving experimental equipment and physiological variations in individual platelets $(86,91,11)$. This technique highlighted the importance of the microtubule coil in maintaining a discoid shape for the platelet, as pharmacological treatment of platelets to remove these microtubules was 
associated with a large increase in platelet extension upon aspiration $(65,66)$. This would suggest that the microtubule coil contributes significantly by exerting a force on the platelet surface to confer platelet resistance to deformation in the micropipette (65). A factor to clarify is the potential for resultant platelet activation following mechanical stimulation, an effect that has thus far proven inconclusive and is therefore a facet of platelet research that demands further analysis. Although limited in experimental support, a study by Haga et al. (11) concluded that platelet activation was not a repercussion of micropipette aspiration, as experimentally the extended platelet tongue re-integrated into the inactive discoid platelet when aspiration ceased. This suggests that the theoretical contributions of the open canalicular system with potentially fused $\alpha$-granules upon platelet activation did not in fact add to membrane extension, as platelet activation was not achieved $(11,66)$.

\subsection{Constitutive Modeling for Platelets}

The study of mechanical disruption causing circulatory trauma has thus far focused almost exclusively on erythrocyte membrane rupture or hemolysis (7), thereby discounting the invaluable role of platelets in regulating hemostatic and thrombotic events. As evidenced in previous sections, the latest blood trauma investigations have identified flow-induced platelet activation as being the primary cause of thrombosis $(37,92)$. This relationship is presumed similar in vivo, with proposed agonists of platelet activation being vascular environments of high shear stress, blood plasma components and damaged vasculature. To better define these models of platelet activation under physiological hemodynamic forces, quantification of the rheological properties of the platelet membrane is desirable. Comprehension of the intrinsic mechanical properties of the platelet plasma membrane will facilitate a better understanding of the implications of platelet deformation in circulation.

Ultimately, the cumulative information regarding platelet activation, platelet deformation, and platelet material properties amount to a more comprehensive understanding of platelet contributions to physiological thrombosis, whether in blood vessels or around MHVs. The following sections (2.5.1-2.5.2) will therefore detail the general mechanism by which the platelet membrane material properties can be determined using constitutive modeling, and present existing models of qualitatively similar cell structures. 


\subsubsection{General Principles and Methods in Constitutive Modeling}

As evidenced in the previous section (Section 2.2) outlining the significance of mechanical stimuli in facilitating biological responses in cellular systems, research has extended this interest to focus on the mechanical properties of soft biomaterials. To this effect, constitutive modeling contributes to a more comprehensive understanding of mechanical influences by providing insight into the mechanical deformation behavior of these unique biological materials.

To achieve an accurate model, one must first summarize the physiological loading conditions and resultant behavior of the tissue/membrane, considering those pathophysiological conditions that may also deform these biological materials. Understanding this physiological deformation range for a biostructure of interest may expose certain model characteristics (i.e. incompressibility) that may facilitate or lengthen the analytical process. Specifically, complexity issues may arise when establishing the scope of the theoretical development, as one can obtain a highly characterized description of a material's stress state using a three-dimensional material model at the expense of computation time (93). Conversely, a more simplistic approach using isotropic material assumptions may fail to adequately capture the inherent complexities of most biological materials. Further, the presence of residual stresses in biological tissue or membrane systems is a common condition (93) that must be identified, as a failure to address this load-free membrane stress would result in an incomplete model (93).

To clarify these uncertainties and identify salient shape transformations, in vivo testing obviously offers direct observation of a material's behavior in its natural mechanical environment, however deformation responses may also be convoluted by unregulated biochemical parameters (93). With this concept of in vivo loading conditions, one can alternatively develop controlled in vitro nonphysiological experimental conditions (for examples, Section $2.4,2.5 .3$ ) that probe the potentially extreme deformation capabilities of the material. Depending on the biomaterial properties of interest, mechanical test designs will vary, such as investigating mechanical properties effected by shear, strain rates (quasistatic or dynamic), or variable loading patterns (cyclically or discontinuously) (93). Notably, the derived material properties vary depending on the mechanism of tissue/cell manipulation 
as well as the accuracy of the theoretical model chosen to fit the resultant experimental data $(94,95)$.

Generally, acceptable constitutive descriptions consider the histological structure of the biological material of interest, outline the main deformation geometries achieved experimentally that are useful or mechanically relevant for the determination of the material's mechanical properties, and identify the general mechanical response characteristics of the material (i.e. isotropic, anisotropic) (93). Following a detailed description of the material's histology, the task of defining the material properties of each relevant component requires extensive and specialized research that is unfortunately dependent on robust experimental data that is scarce (93). One must therefore appreciate that most constitutive models fail to directly address the complexities of histological structure by taking a continuum mechanics approach, and therefore use a phenomenological model that describes a biostructure in macroscopic terms (93).

Essentially, this development begins with a theoretical framework that accurately describes the fundamental mechanical behavior of the material in response to an idealized loading state (96). It is therefore advantageous to use a theory that accounts for the finite deformations and nonlinear responses (hyperelastic behavior) inherent to many soft biomaterials $(98,94,99)$. Compatible with this behavior, equations that describe the experimentally observed stress-strain relationship of a specific biological material are derived from a constitutive equation and material constants that reliably predict the deformation state $(93,96)$. The most common of such functions (ex. strain energy function) are exponential in nature, however logarithmic and polynomial forms have also garnered attention (93). These general equations are then tailored to address the geometric features of the biostructure that are relevant for the analysis of subsequent deformation responses (ex. extension, torsion, compression, inflation) that this system will incur (93).

Overall, it is important to take a critical approach to constitutive modeling, exploring those previous modeling attempts which may have had certain "mechanical, mathematical and computational deficiencies" (93) so as to formulate a novel model that provides a more comprehensive and accurate description of biomaterial mechanical behaviors. 


\subsubsection{A Constitutive Theory for Biological Membranes}

Along the lines of general constitutive modeling of biological materials, Humphrey et al. (97) sought to develop a constitutive relationship for any nonlinear biological membrane, as defined by a structurally thin shell which is assumed to have negligible resistance to bending following finite deformations. Further, this theoretical development assumes that in-plane stresses $^{1}$ do not vary significantly across membrane thickness. Considering the membrane as a two-dimensional continuum, Humphrey et al. (97) details the conversion of a threedimensional strain energy function, $\bar{W}$, to one that describes membrane material behavior in two-dimensions. Ultimately, the two-dimensional strain energy function, $W$, is defined per initial surface area of the material, rather than volume (97).

For a general membrane deformation in three-dimensions, the in-plane components of the deformation gradient tensor, $\overline{\bar{F}}$, are represented in matrix form (97):

$$
[\overline{\bar{F}}]=\left[\begin{array}{ccc}
F_{11} & F_{12} & 0 \\
F_{21} & F_{22} & 0 \\
0 & 0 & \lambda_{3}
\end{array}\right] \quad \text { where } \lambda_{3}=\frac{h}{H}
$$

Essentially, each deformation gradient tensor component characterizes the displacement of a material point with initial position vector ' $X$ ' to a deformed configuration ' $x$ '. Also, the extension ratio, $\lambda_{3}$, describes the relationship between the membrane's initial thickness, $H$, and current thickness, $h$, following deformation. Thus, the Jacobian determinant of $\overline{\bar{F}}$ is evaluated to produce the following:

$$
J=\operatorname{det} \overline{\bar{F}}=\lambda_{3}\left(F_{11} F_{22}-F_{12} F_{21}\right),
$$

which can be alternatively expressed to relate the current membrane material volume $(V)$, and the initial membrane volume $\left(V_{\mathrm{o}}\right)$ :

$$
J=\frac{V}{V_{o}}=\frac{h}{H} J_{2 D} .
$$

Ultimately, Humphrey et al. (97) defines a general three-dimensional relationship for a hyperelastic material which relates the Cauchy stress tensor, $\bar{t}$, to the strain energy function, $\bar{W}$, as

\footnotetext{
1 ' 1,2 ' directional subscripts denote in-plane components, whereas the out-of-plane component is denoted by the subscript ' 3 '.
} 


$$
\bar{t}=\frac{2}{J} \overline{\bar{F}} \frac{\partial \bar{W}}{\partial \overline{\bar{C}}} \overline{\bar{F}}^{\mathbf{T}}, \quad \text { with } \quad \overline{\bar{C}}=\overline{\bar{F}}^{\mathrm{T}} \overline{\bar{F}},
$$

where $\overline{\bar{C}}$ is the right Cauchy-Green deformation tensor. Following this three-dimensional development, the equation is recast for membranes introducing a two-dimensional strain energy function, $W$ :

$$
\begin{aligned}
J t & =2 \overline{\bar{F}} \frac{\partial \bar{W}}{\partial \overline{\bar{C}}} \overline{\mathrm{F}}^{T} \\
\frac{h}{H} J_{2 D} t & =2 \overline{\bar{F}} \frac{\partial \bar{W}}{\partial \overline{\bar{C}}} \overline{\mathrm{F}} \\
\bar{t}_{2 D} & =\frac{2}{J_{2 D}} \overline{\bar{F}}_{2 \mathrm{D}} \frac{\partial W}{\partial \overline{\bar{C}}_{2 D}} \overline{\overline{\mathrm{F}}}_{2 D}{ }^{T} \quad \text { with } \overline{\mathrm{W}}\left(\overline{\bar{C}}_{3 D}\right)=\frac{W\left(\bar{C}_{2 D}\right)}{H} \\
\overline{\bar{T}} & =\frac{2}{J_{2 D}} \overline{\bar{F}}_{2 \mathrm{D}} \frac{\partial W}{\partial \overline{\bar{C}}_{2 D}} \overline{\mathrm{F}}_{2 D}{ }^{T}
\end{aligned}
$$

where $\overline{\bar{T}}$ is a general in-plane stress resultant tensor ${ }^{2}$ with nonspecific membrane applications. Considering only principal directions, the two-dimensional gradient tensor, $\overline{\bar{F}}_{2 D}$, is expressed in terms of extension ratios, $\lambda_{1}$ and $\lambda_{2}$, which define the ratios between the final $\left(d y_{1}, d y_{2}\right)$ and initial $\left(d x_{1}, d x_{2}\right)$ lengths of a deformed biomembrane surface element:

in addition,

$$
\left[\overline{\bar{F}}_{2 D}\right]=\left[\begin{array}{ll}
\lambda_{1} & 0 \\
0 & \lambda_{2}
\end{array}\right] \quad \text { with } J_{2 D}=\operatorname{det} \overline{\bar{F}}_{2 D}=\lambda_{1} \lambda_{2},
$$

$$
\left[\frac{\partial \mathrm{W}}{\partial \overline{\overline{\mathrm{C}}}_{2 D}}\right]=\left[\begin{array}{cc}
\frac{\partial \mathrm{W}}{\partial C_{11}} & 0 \\
0 & \frac{\partial \mathrm{W}}{\partial C_{22}}
\end{array}\right]
$$

Ultimately, membrane stresses in these principal directions can be expressed as,

$$
\begin{aligned}
& T_{11}=\frac{1}{\lambda_{2}} \frac{\partial W}{\partial \lambda_{1}} \\
& T_{22}=\frac{1}{\lambda_{1}} \frac{\partial W}{\partial \lambda_{2}} .
\end{aligned}
$$

\footnotetext{
${ }^{2}$ A two-dimensional measure of in-plane force per unit in-plane length. As such, this in-plane stress resultant is not a stress, but is often referred to as a membrane stress or tension.
} 


\subsubsection{Existing Models Inspired from Erythrocyte Membrane Models}

As mentioned, a current focus of cellular research investigates the continuum mechanical properties of soft biomaterials, of which the derivation by Humphrey (97) offers a constitutive theory useful for generalized biomembrane applications. Maintaining relevance to the current work, it is important to characterize existing membrane models to use as a theoretical framework from which to build. As such, the investigation of existing erythrocyte membrane models possesses obvious advantages, offering a well-characterized, structurally similar cell to platelets which endures similar in vivo hemodynamic conditions (see Section 2.2.1).

Based on well-characterized shape transformations of erythrocytes in response to single-cell manipulations including cell-sphering (94), preliminary membrane research by Fung and Tong (101) implemented mechanical equilibrium equations for an axisymmetric membrane, and defined their own isotropic, elastic stress-strain relationship to describe erythrocyte membrane behavior for finite deformations. Under these deformation conditions, bending stresses are also assumed negligible relative to membrane stresses, as the erythrocyte membrane is considered a thin shell (98). Although this stress-strain law failed to accurately describe experimentally observed membrane deformation behavior, Fung and Tong (101) demonstrated the feasibility of erythrocyte membrane constitutive modeling with experimental validation.

Developing this research pursuit further, Evans (98) and Skalak et al. (94) sought to apply Humphrey's (97) rationale to erythrocyte membranes, proposing alternative forms for the strain energy function, $W$, in two-dimensions. One must appreciate that these developments take a continuum-based approach and pertain to a geometrically simple cell evaluated at the whole-cell or global level, modeling an erythrocyte composition that naturally lacks a reinforcing cytoskeleton. Both models also propose an elastic material membrane, and formulate stress-strain laws for finite deformations that describe membrane behavior following the aforementioned sphering transformation (98). For simplicity, however, subsequent theoretical developments are individually presented.

Using similar conventions to Humphrey (97), Evans (98) introduces a twodimensional Cauchy-Green deformation tensor, $\overline{\bar{C}}_{2 D}$, 


$$
\left[\overline{\bar{C}}_{2 D}\right]=\left[\begin{array}{cc}
\lambda_{1}^{2} & 0 \\
0 & \lambda_{2}^{2}
\end{array}\right]
$$

where the first invariant of this two-dimensional tensor is described by,

$$
I_{C_{2 D}}=\operatorname{tr} \overline{\bar{C}}_{2 D}=\lambda_{1}^{2}+\lambda_{2}^{2} \text {. }
$$

Next, the development by Evans (98) treats the erythrocyte membrane as a nearly incompressible material by citing osmotic-sphering, which demonstrated negligible surface area increases following cellular deformation. Similarly, a study by Hochmuth et al. (102) testing shear-deformed fixed cells demonstrated two-dimensional incompressibility, as the product of the two extension ratios yields the characteristic magnitude of one. Satisfying this incompressibility constraint (i.e. $\lambda_{1} \lambda_{2}=1$ ) for a two-dimensional material, the adapted derivation of the in-plane stress resultant tensor, $\overline{\bar{T}}$, also includes a lagrangian multiplier, $p_{\mathrm{h}}$, to yield the following:

$$
\overline{\bar{T}}=-p_{h} \overline{\bar{I}}+2 \frac{\partial W}{\partial I_{C_{2 D}}} \overline{\bar{C}}_{2 D} \quad \text { with } W=W_{o}+\mathrm{c}_{1}\left(I_{C_{2 D}}-2\right) .
$$

The two-dimensional strain energy function, $W$, describes small strain membrane responses, while the lagrangian multiplier is considered an analogue to hydrostatic pressure (98).

Subsequently, the principal membrane stresses are as follows:

$$
\begin{aligned}
& T_{1}=-p_{h}+2 c_{1} \lambda_{1}^{2} \\
& T_{2}=-p_{h}+2 c_{1} \lambda_{2}^{2} .
\end{aligned}
$$

Unfortunately, these constitutive descriptions have limited applicability to erythrocytes maintaining spherical geometries (i.e. $T_{1}=T_{2}$ ), as this condition is only achieved when the incompressible membrane does not stretch in either principal direction (i.e. $\lambda_{1}=\lambda_{2}=1$ ).

As an alternative, Skalak et al. (94) maintain the same gradient tensor, $\overline{\bar{C}}_{2 D}$, as Evans (98), but propose two separate invariants $\left(I_{1}, I_{2}\right)$ to complement his own two-dimensional study of erythrocyte membranes:

$$
\left[\overline{\bar{C}}_{2 D}\right]=\left[\begin{array}{cc}
\lambda_{1}^{2} & 0 \\
0 & \lambda_{2}^{2}
\end{array}\right] \text { with } I_{1}=\lambda_{1}^{2}+\lambda_{2}^{2}-2 \text { and } I_{2}=\lambda_{1}^{2} \lambda_{2}^{2}-1=\left(\frac{d A}{d A_{o}}\right)^{2}-1
$$

where $d A_{\mathrm{o}}$ and $d A$ are the initial and final areas of a surface membrane element. Skalak et al. (94) also postulate an alternative two-dimensional strain energy function, 


$$
W=\frac{c_{1}}{4}\left(\frac{1}{2} I_{1}^{2}+I_{1}-I_{2}\right)-\frac{c_{2}}{8} I_{2}^{2} .
$$

Similar to Evans (98), this strain energy function enables a description of erythrocyte membrane deformation behavior, by defining two membrane material constants, $c_{1}$ and $c_{2}$. With further reduction, the principal membrane stresses are given by:

$$
\begin{aligned}
& T_{1}=2 \frac{\lambda_{1}}{\lambda_{2}}\left[\frac{\partial W}{\partial I_{1}}+\lambda_{2}^{2} \frac{\partial W}{\partial I_{2}}\right] \\
& T_{2}=2 \frac{\lambda_{2}}{\lambda_{1}}\left[\frac{\partial W}{\partial I_{1}}+\lambda_{1}^{2} \frac{\partial W}{\partial I_{2}}\right] .
\end{aligned}
$$

Once again, maintaining a spherical geometry where tension values are directionally equivalent (i.e. $T_{1}=T_{2}$ ) is only possible when the strain energy function is dependent on the single invariant, $I_{2}$ [i.e. $W=W\left(I_{2}\right)$ ]. In contrast, incorporating both strain invariants establishes a geometric condition where the deformed cell could only assume a spherical shape when $c_{1} \equiv 0$, generating the following constitutive relation,

$$
T_{1}=T_{2}=\frac{\lambda_{1} \lambda_{2}}{2}\left(1-\lambda_{1}^{2} \lambda_{2}^{2}\right)
$$

To complement the development by Evans (98), conserving areal strain $\left(\lambda_{1} \lambda_{2}=1\right)$ in the absence of membrane stretch along the principal directions (i.e. $\lambda_{1}=\lambda_{2}=1$ ) would indicate an absence of membrane stresses (i.e. $T_{1}=T_{2}=0$ ).

Evidently, these erythrocyte models establish the feasibility of constitutive modeling using novel strain energy functions for single cell membrane applications. In presuming erythrocyte membrane incompressibility, however, such models are limited in their capacity to accurately model the membrane mechanical behavior of cells assuming a spherical shape during deformation. 


\section{Chapter 3}

\section{Experiments}

As platelets are recognized for their intrinsic sensitivity and small size, a modernized attempt to verify the feasibility of micropipette aspiration using murine platelets proves advantageous. The following chapter therefore describes the various mechanisms of sample collection, processing and manipulation that contribute to a novel set of deformation data for the murine platelet.

\subsection{Materials and Methods}

\subsubsection{Sample Collection Overview}

When attempting to characterize the functional and physical properties of platelets by means of in vitro platelet assays, it is imperative to preserve the inactive integrity of blood following collection (46). Particularly for mice, their small size and limited blood volume $(\sim 1.8 \mathrm{~mL}$ in an average mouse of $\sim 25 \mathrm{~g}$ ) increases the probability of platelet activation during the blood withdrawal process (46). The withdrawal of $0.5-0.7 \mathrm{~mL}$ of blood from adult mice is well tolerated, with the advantage of a relatively fast recovery time (within 24 hours) (103). Blood can be collected by a number of mechanisms, such as through the "retobulbar venous plexus ("retroorbital"), heart, inferior vena cava, jugular vein, carotid artery, tail or lateral saphenous veins" (46). Each entry point confers variability in terms of method, blood volume yield, platelet activation and animal risk. Notably, blood collection through puncturing of the inferior vena cava can generate a $1-2 \mathrm{~mL}$ sample and is recognized to minimize the incidence of platelet activation, but requires surgical exposure of the vein and subsequent use of a 22 - 27 gauge needle $(42,46)$. A similar volume is also achieved with cardiac puncture, which is a terminal procedure where cervical dislocation is performed following blood collection and is recognized to have minimal risk in causing platelet activation (104). 


\subsubsection{Animals}

Mice used for blood collection were $P g f^{+/+}$or $P g f^{-\gamma_{-}}$of both genders and range in size from 28-30 grams. Mouse models with polar expression (i.e. wild type or knockout) of the PlGF gene do not contribute significantly to proper platelet function, rather the PlGF gene contributes to pathological angiogenesis and adaptive arteriogenesis (105). Blood was collected from isoflurane-anaesthetized mice by cardiac puncture using a $1 \mathrm{cc}$ syringe with a 25 gauge needle. Isoflurane was obtained from Webster Veterinary (Sterling, MA) and is recognized to have negligible effects on platelet function in vitro at therapeutic concentrations (Induction, 4\% anesthetic vaporization; Maintenance, $1.5-3 \%$ anesthetic vaporization) (See Appendix A) (106). With a clean draw, an average volume collected from each mouse ranged between $0.7-1.0 \mathrm{~mL}$, which was immediately pooled ( 3 - 5 mice) and combined with an anticoagulant known to minimize the incidence of platelet activation (107, 46). Typically, sodium citrate is implemented in studies where platelet function is analyzed in plasma, while acid citrate dextrose is advantageous when preparing washed or gel-filtered platelets (46). It is also recognized that the presence of ADP and collagen in plasma will induce greater platelet aggregation in heparinized PRP rather than citrated PRP (108). As such, a 3.2\% buffered sodium citrate solution containing sodium citrate, theophylline, adenosine and dipyridamole in a ratio of 5 parts blood to 1 part anticoagulant within a CTAD vacutainer was used (109). Agents such as dipyridamole are commonly used to inhibit platelet aggregation in human blood samples, and are also applicable to murine platelet assays $(42,110)$.

\subsubsection{Washed Platelet Preparation}

The accepted standard for human platelet preparation from White et al. (65) has been adjusted to accommodate the divergent demands of mouse platelets outlined above (Section 3.1.1). As mentioned, the platelet yield from each mouse is significantly less than that of human donors, which often encourages the pooling of blood collected from several animals $(\sim 3-15$ animals) so that a sufficient number of platelets is collected (46). Following this collection, most assays isolate platelets from whole blood to yield platelet rich plasma (PRP), with a potential for additional purification using a washing or gel-filtration technique (46). All manipulations (storage, handling) are performed at room temperature, and are performed 
using polyethylene plastic, polypropylene plastic, polycarbonate plastic, or siliconized glass (107). The isolation specifications for platelet rich plasma varies depending on the methodology, but implements either a single centrifugation or a multiple-step centrifugation process which isolates platelet populations from the plasma and the buffy coat (46). Murine platelets are not easily separated from other cellular components (erythrocytes and leukocytes) when compared to human platelets under gentle centrifugal forces, as murine platelets occur at a larger population density in whole blood (42). As such, an adequate sample of murine platelet-rich plasma is difficult to isolate, and may therefore require the application of larger centrifugal forces (104) and subsequent addition of a buffer (i.e. Tyrode's buffer). This washing step reconstitutes platelets within the liquid medium and eliminates those procoagulant plasma enzymes (i.e. thrombin) generated during whole blood collection and preparation, while also providing a highly controlled solution of inorganic ions, proteins and platelet aggregation antagonists (104). Buffers should also be controlled for $\mathrm{pH}$ (range, 7.2 - 7.4), as any divergence from the physiological range may alter platelet function (104).

Following blood collection the CTAD blood sample settled for 15 minutes, whereupon platelet rich plasma was isolated from the whole blood by centrifugation (model GS-15R, Beckman Coulter, Mississauga, ON) at 3716 RPM $(2300 \times \mathrm{g})(10 \mathrm{~s} / \mathrm{mL})$ at room temperature $\left(\sim 22^{\circ} \mathrm{C}\right)(104)$. During this density separation technique, braking of the centrifuge was avoided. At the same time, a $15 \mathrm{~mL}$ polypropylene conical centrifuge tube labeled 'PRP' was coated with HEPES-modified $\mathrm{Ca}^{2+}$-free Tyrodes buffer, pH 7.3 (138 mM $\mathrm{NaCl}, 2.7 \mathrm{mM} \mathrm{KCl}, 2 \mathrm{mM} \mathrm{MgCl} 6 \mathrm{H}_{2} 0,12 \mathrm{mM} \mathrm{NaHCO}_{3}, 0.4 \mathrm{mM} \mathrm{Na}_{2} \mathrm{HPO}_{4}, 1 \%$ bovine serum albumin, $10 \mathrm{mM}$ HEPES, $5.5 \mathrm{mM}$ dextrose), that was kindly donated by Dr. Antonio Giulivi, Ottawa General Hospital, Ottawa, ON. Following the first phase of centrifugation, samples of PRP were transferred to the labeled conical tube and mixed with an equal volume of 3.8\% sodium citrate anticoagulant, $\mathrm{pH} 8.0$ (RICCA chemical company, Arlington, TX) and centrifuged at $3634 \mathrm{RPM}(2200 \mathrm{x} \mathrm{g})$ for 4 minutes to pellets at room temperature (107, 104). Supernatant was discarded and the platelet-rich pellet was recombined with approximately $0.7 \mathrm{~mL}$ of filtered HEPES-modified $\mathrm{Ca}^{2+}$-free Tyrodes buffer without disturbing the pellet. The buffer was aspirated immediately and discarded. Addition of this buffer to the pellet will remove the $\mathrm{Ca}^{2+}$ from the suspension, which reduces the potential for 
activation (16). An additional $1.4 \mathrm{~mL}$ of buffer was then added to gently resuspend the pellet without introducing air bubbles. Theophylline $(3 \mathrm{mM})$ and adenosine $(5 \mathrm{mM})$ (Acros Organics, Fair Lawn, NJ) were also incubated with the suspending buffer to impede potential platelet activation upon contact with foreign surfaces (Becton, Dickinson and Company, 2008). Washed platelets were immediately implemented in micropipette aspiration experiments performed within three hours of blood collection at room temperature (104).

\subsubsection{Micropipette Aspiration and Imaging Procedure}

Micropipettes were prepared in a one-step process using a horizontal micropipette puller (model P-80, Sutter Instrument Co., Novato, CA). The thin-walled boron silicate glass capillary pipettes with filaments (World Precision Instruments Inc., Sarasota, FL), $1.5 \mathrm{~mm}$ in external diameter, were heated and drawn in the puller to assume needle-like structures. To maintain micropipette durability and ensure an appropriate taper length, the micropipette puller was fixed with a $2.5 \mathrm{~mm} \times 2.5 \mathrm{~mm}$ box filament (111). These micropipettes tips have a fairly short taper $(\sim 4-6 \mathrm{~mm})$ and have internal diameters appropriate for platelet studies $(\sim 0.6-1.4 \mu \mathrm{m})$. These internal diameters were observed and measured with an upright microscope (Fisher Scientific, Pittsburgh, PA) equipped with an objective micrometer. The implementation of filamented glass to produce micropipettes with tip diameters within this range ensured easy filling of the Tyrode's buffer solution into the pipette. Coupled to a large plastic syringe via plastic tubing and fixed to a micropipette holder, a small magnitude of positive pressure was applied through the micropipette tip to prevent tip blockage as it first crossed the meniscus of the filtered buffer. Negative pressure was then applied through the immersed micropipette tip until the majority of the short taper was filled with buffered solution. The remaining large diameter portion of the pipette was backfilled using a thin wire guide (MicroFil 28g, World Precision Instruments Inc., Sarasota, FL) coupled with a filter unit (MILLIPORE, Bedford, MA) to prevent pipette blockage. Any air bubbles trapped within this filled pipette were removed prior to micropipette use. Filled pipettes were secured in a microelectrode holder (World Precision Instruments Inc., Sarasota, FL) with a pressure-sealed screw cap and were fixed in a micromanipulator (model MP-285, Sutter Instrument Co., Novato, CA). This unit was connected to a pressure adjustment system and a water manometer by buffer-filled rigid tubing made of polyethylene plastic, which is free 
from trapped air. The pressure adjustment system consisted of a screw-driven syringe that exerted negative and positive pressure through the micropipette. Joints in this pressure system were sealed with parafilm (' $M$ ' laboratory film, American National Can Company, Chicago, IL) so as to guard against potential leaks.

Approximately $0.7 \mathrm{~mL}$ of washed platelets were placed on a $12 \mathrm{~mm}$ siliconized glass cover slip fixed to a specimen chamber and mounted on the stage of an upright microscope (Axioskop FS, Carl Zeiss Inc., Toronto, ON) with phase-contrast optics. Platelets were observed through a Zeiss 100x water dipping objective lens (AchroPlan 100x/1.0 W Ph3, Toronto, $\mathrm{ON}$ ) and a 10x eyepiece. A split prism transmitted the resultant cellular image to a video camera (model XCD- X700, SONY, Toronto, ON) that transmits and records the image in the Northern Eclipse Imaging program (Version 7.0) where it is visualized on a computer monitor. The microscope and micromanipulator were supported on a hydraulic anti-vibration table (Technical Manufacturing Corporation, Peabody, MA), while the negative pressure generator was located directly adjacent to this system.

The tip of the pipette was carefully positioned over the sample under direct observation, while a constant positive pressure of $1 \mathrm{~cm} \mathrm{H}_{2} \mathrm{O}$ was maintained during sample immersion through the meniscus. To minimize tip blockage, this technique was maintained until the pipette tip approached the surface of a platelet, whereupon a negative pressure of 1 $\mathrm{cm} \mathrm{H}_{2} \mathrm{O}$ was applied to ensure contact. Only discoid platelets were used for aspiration studies. Once in contact, successive increases in negative pressure of $1 \mathrm{~cm} \mathrm{H}_{2} \mathrm{O}$ were applied until a maximum pressure of $10 \mathrm{~cm} \mathrm{H}_{2} \mathrm{O}$ was reached. Any adherence of the platelet to the micropipette was assumed negligible, and only those platelets that passively detached from the pipette after suction were used. The aspiration lengths $\left(L_{\mathrm{p}}\right)$ of platelets at each successive pressure $\left(p_{\mathrm{i}}\right)$ were recorded once the aspirated projection reached equilibrium using the Northern Eclipse imaging program. In addition, the micropipette tip radius $\left(R_{\mathrm{p}}\right)$ and the major and minor radii of the unaspirated platelet portion were recorded.

To calibrate the magnification of the microscope system and each micropipette internal diameter, calibration images of known dimensions corresponding to given magnifications were inputted into the Northern Eclipse imaging program. The lengths of these images under a magnification of $100 \mathrm{x}$ water dipping objective lens (AchroPlan $100 \mathrm{x} / 1.0 \mathrm{~W} \mathrm{Ph} 3$, Toronto, ON) were previously measured using a vernier caliper and 
inputted into the Northern Eclipse calibration program. Values were expressed in micrometers.

\subsection{Results}

\subsubsection{Aspiration Measurements of Inactive Murine Platelet Membranes}

To visualize the effects of micropipette aspiration on the murine platelet, Figure 3.1 depicts the typical membrane response to a controlled gradient of negative pressure.

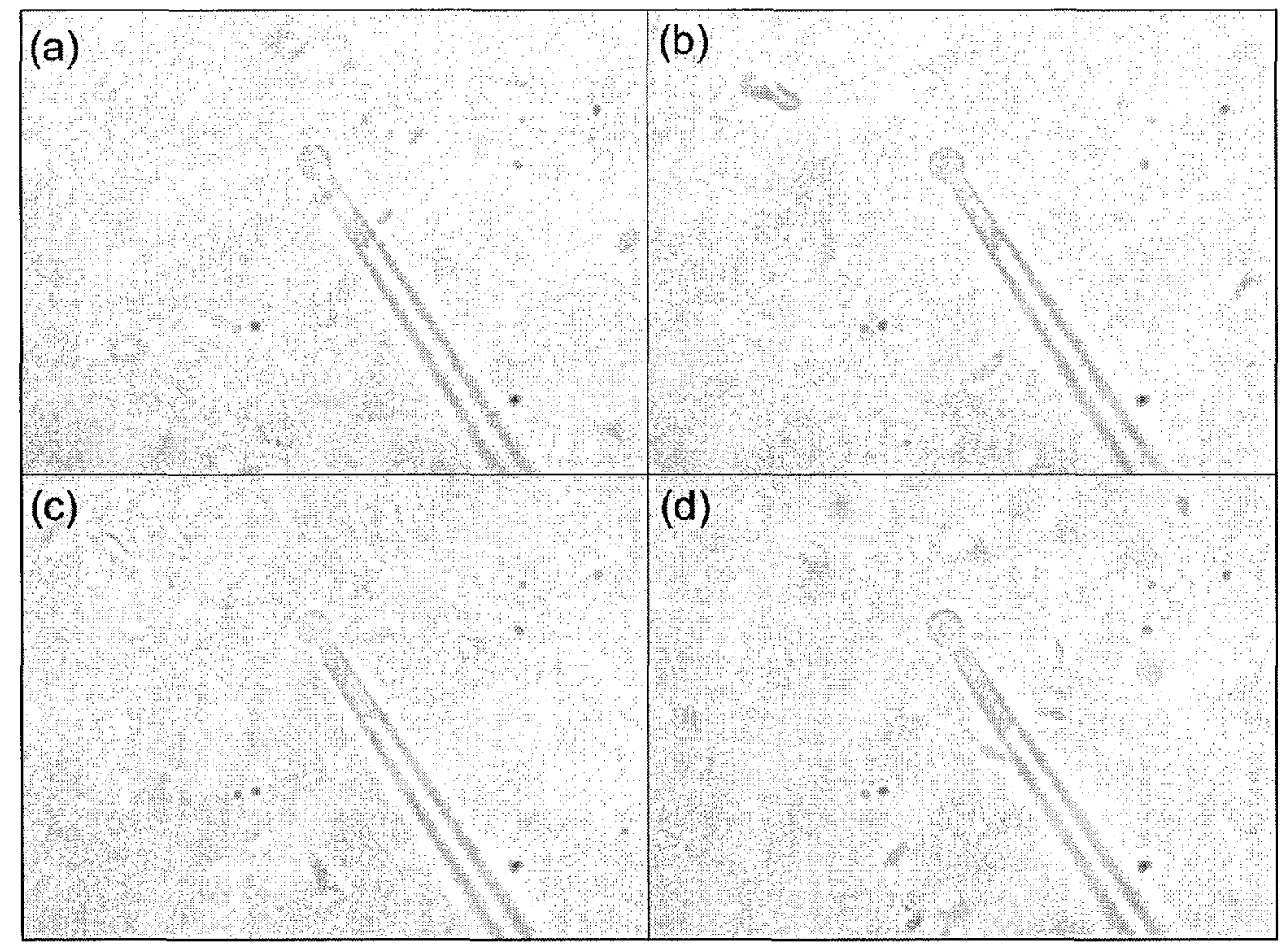

Figure 3.1: Micropipette aspiration of a murine platelet. A single resting platelet was aspired over a pressure gradient of $1-4 \mathrm{~cm} \mathrm{H} \mathrm{H}_{2} \mathrm{O}\left(\sim 98-392 \mathrm{~N} / \mathrm{m}^{2}\right)$ (Figures 3.1, a-d).

Table 3.1 presents the salient information from each micropipette aspiration experiment of single inactive murine platelets. Inactive platelet deformation behavior was quantified in terms of resultant aspirated platelet length $\left(L_{\mathrm{p}}\right)$ and magnitudes of the major radius $(\mu \mathrm{m})$ and minor radius $(\mu \mathrm{m})$ of the unaspirated platelet fraction. The average pipette radius $\left(R_{\mathrm{p}}\right)$ was also recorded, along with observed cases of platelet lysis. Although static deformation data 
was collected, overt signs of membrane viscoelasticity (strain dependent on time) were absent.

Table 3.1: Aspiration measurements of individual murine platelet membranes. The average shape ratio (major radius/minor radius) of the unaspirated platelet fraction was calculated by taking measurements over the entire aspiration pressure range for each sampled platelet.

\begin{tabular}{|c|c|c|c|c|c|c|c|}
\hline $\begin{array}{c}\text { Aspiration } \\
\text { Pressure } \\
\left(\mathrm{N} / \mathrm{m}^{2}\right)\end{array}$ & $L_{\mathrm{p}}(\mu \mathrm{m})$ & $\begin{array}{c}\text { Average } \\
R_{\mathrm{p}}(\mu \mathrm{m})\end{array}$ & $\begin{array}{c}\text { Average } \\
\text { major/minor (-) }\end{array}$ & $\begin{array}{c}\text { Aspiration } \\
\text { Pressure }\left(\mathrm{N} / \mathbf{m}^{2}\right)\end{array}$ & $L_{\mathrm{p}}(\mu \mathrm{m})$ & $\begin{array}{c}\text { Average } \\
R_{\mathrm{p}}(\mu \mathrm{m})\end{array}$ & $\begin{array}{c}\text { Average } \\
\text { major/minor (-) }\end{array}$ \\
\hline \multicolumn{4}{|c|}{ Platelet 1} & \multicolumn{4}{|c|}{ Platelet 10} \\
\hline 0.0 & & \multirow[t]{4}{*}{0.68} & \multirow[t]{4}{*}{0.99} & 0.0 & 3.50 & \multirow[t]{4}{*}{0.39} & \multirow[t]{4}{*}{1.08} \\
\hline 98.1 & 6.77 & & & 98.1 & 3.92 & & \\
\hline 196.1 & 6.70 & & & 196.1 & 14.33 & & \\
\hline 294.2 & 7.02 & & & 294.2 & 20.02 & & \\
\hline \multicolumn{4}{|c|}{ Platelet 2} & \multicolumn{4}{|c|}{ Platelet 11} \\
\hline 0.0 & 7.28 & \multirow[t]{5}{*}{0.36} & \multirow[t]{5}{*}{1.04} & 0.0 & & \multirow[t]{3}{*}{0.50} & \multirow[t]{3}{*}{1.06} \\
\hline 98.1 & 6.50 & & & 98.1 & 9.18 & & \\
\hline 196.1 & 7.51 & & & 196.1 & 10.58 & & \\
\hline 294.2 & 8.30 & & & \multicolumn{4}{|c|}{ Platelet 12} \\
\hline 392.3 & 9.33 & & & 0.0 & & \multirow[t]{6}{*}{0.33} & \multirow[t]{6}{*}{1.03} \\
\hline \multicolumn{4}{|c|}{ Platelet 3} & 98.1 & 4.39 & & \\
\hline 0.0 & 5.15 & 0.32 & 1.05 & 196.1 & 5.32 & & \\
\hline 98.1 & 8.59 & & & 294.2 & 5.81 & & \\
\hline 196.1 & 10.08 & & & 392.3 & 8.55 & & \\
\hline 294.2 & 12.52 & & & 490.3 & 9.83 & & \\
\hline \multicolumn{4}{|c|}{ Platelet 4} & \multicolumn{4}{|c|}{ Platelet 13} \\
\hline 0.0 & 3.84 & 0.36 & 1.03 & 0.0 & 3.65 & 0.33 & 1.06 \\
\hline 98.1 & 8.55 & & & 98.1 & 7.76 & & \\
\hline 196.1 & 9.90 & & & 196.1 & 10.28 & & \\
\hline 294.2 & 11.93 & & & 294.2 & 13.61 & & (cell lysed) \\
\hline \multicolumn{4}{|c|}{ Platelet 5} & \multicolumn{4}{|c|}{ Platelet 14} \\
\hline 0.0 & 5.75 & 0.37 & 1.05 & 0.0 & 1.29 & 0.33 & 1.02 \\
\hline 98.1 & 7.51 & & & 98.1 & 6.65 & & \\
\hline 196.1 & 8.17 & & & 196.1 & 8.46 & & \\
\hline 294.2 & 8.50 & & & 294.2 & 9.01 & & \\
\hline \multicolumn{4}{|c|}{ Platelet 6} & 392.3 & 11.00 & & \\
\hline 0.0 & & 0.54 & 1.09 & 490.3 & 13.09 & & \\
\hline 98.1 & 13.74 & & & 588.4 & 13.45 & & \\
\hline 196.1 & 19.15 & & & 686.5 & 13.18 & & $(\mathrm{cel1})(\mathrm{sed})$ \\
\hline 294.2 & 23.04 & & & & & let 15 & \\
\hline & & elet 7 & & 0.0 & 4.56 & 0.33 & 1.03 \\
\hline 0.0 & 7.55 & 0.51 & 1.05 & 98.1 & 7.46 & & \\
\hline 98.1 & 13.22 & & & 196.1 & 9.13 & & \\
\hline 196.1 & 17.16 & & & 294.2 & 9.73 & & \\
\hline 294.2 & 20.97 & & & 392.3 & 13.32 & & \\
\hline 392.3 & 23.63 & & & 490.3 & 14.42 & & \\
\hline 490.3 & 26.43 & & & 588.4 & 14.53 & & \\
\hline & & elet 8 & & & & let 16 & \\
\hline 0.0 & 3.76 & 0.34 & 1.04 & 0.0 & & 0.33 & 1.01 \\
\hline 98.1 & 13.74 & & & 98.1 & 7.44 & & \\
\hline 196.1 & 17.32 & & & 196.1 & 11.09 & & \\
\hline 294.2 & 19.02 & & & & & let 17 & \\
\hline & & elet 9 & & 0.0 & 3.46 & 0.30 & 1.05 \\
\hline 0.0 & 4.66 & 0.42 & 1.04 & 98.1 & 7.51 & & \\
\hline 98.1 & 12.97 & & & 196.1 & 9.94 & & \\
\hline 196.1 & 13.76 & & & & & & \\
\hline 294.2 & 14.82 & & & & & & \\
\hline
\end{tabular}


In response to micropipette aspiration, Figure 3.2 displays individual membrane deformation behavior for 17 inactive platelets, describing a linear increase in extension length over a pressure range of 0 to $7 \mathrm{~cm} \mathrm{H} \mathrm{H}_{2} \mathrm{O}\left(\sim 0-686 \mathrm{~N} / \mathrm{m}^{2}\right)$. This relationship is represented by plotting the product of aspiration pressure $\left(p_{\mathrm{i}}\right)$ and pipette tip radius $\left(R_{\mathrm{p}}\right)$ versus the quotient of aspired platelet length $\left(L_{\mathrm{p}}\right)$ and pipette tip radius. Notably, the y-axis is defined as such to normalize $L_{\mathrm{p}}$ values obtained using micropipettes of various internal radii (86). As later emphasized in Section 4.5.2, only platelets that exhibit this linear deformation behavior while maintaining constant volume are assumed significant and useful for subsequent characterization of platelet membrane material properties. As such, the average deformation behavior for inactive platelet membranes (mean $\pm \mathrm{SD}$, for 11 platelets) is defined in Figure 3.3. Each mean was based on measurements of 11 aspirated platelets proven to maintain a constant volume over the entire range of negative pressure.

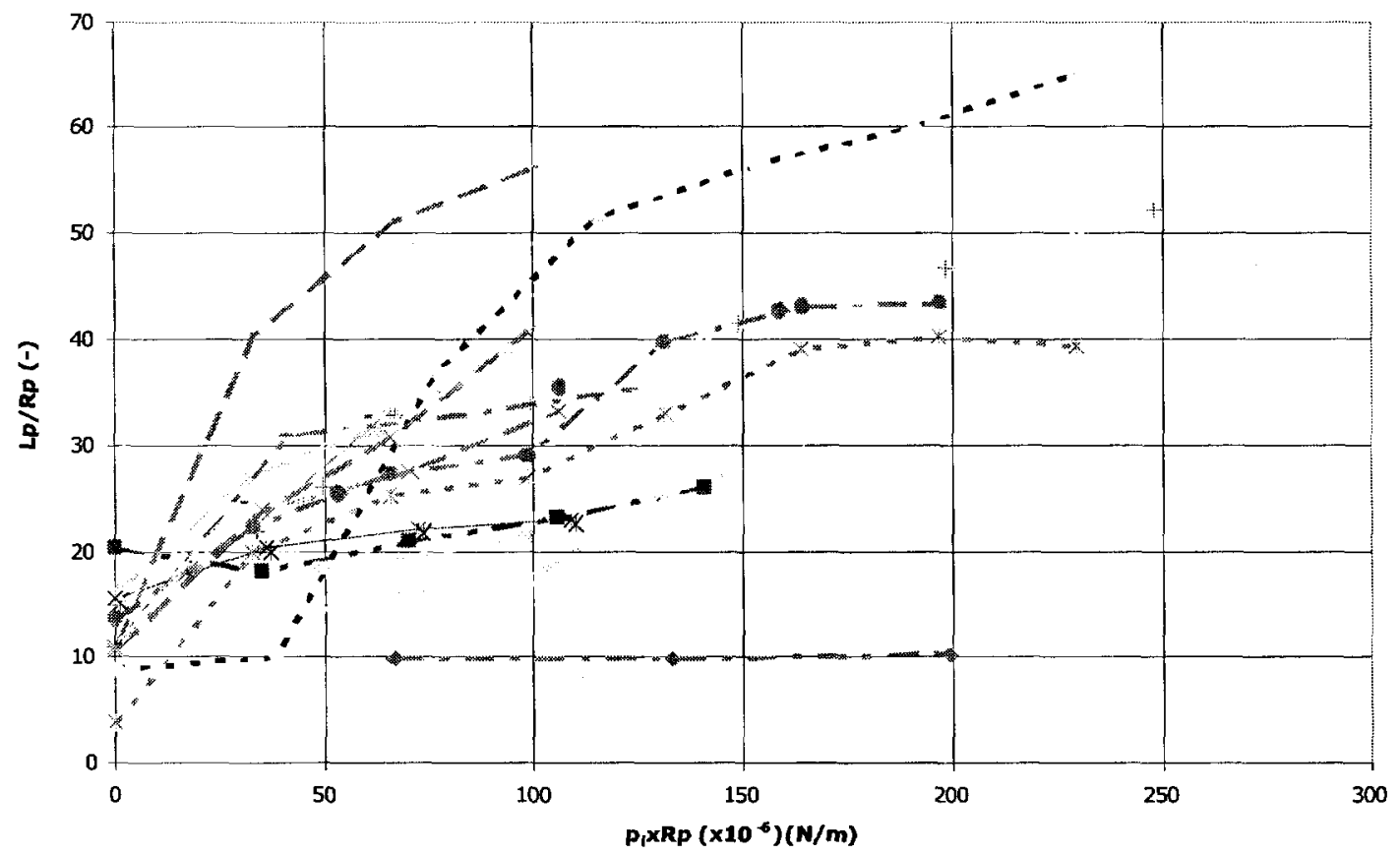

Figure 3.2: Deformation behavior of individual aspirated murine platelet membranes. To improve legibility, variable line styles were introduced for each platelet. 


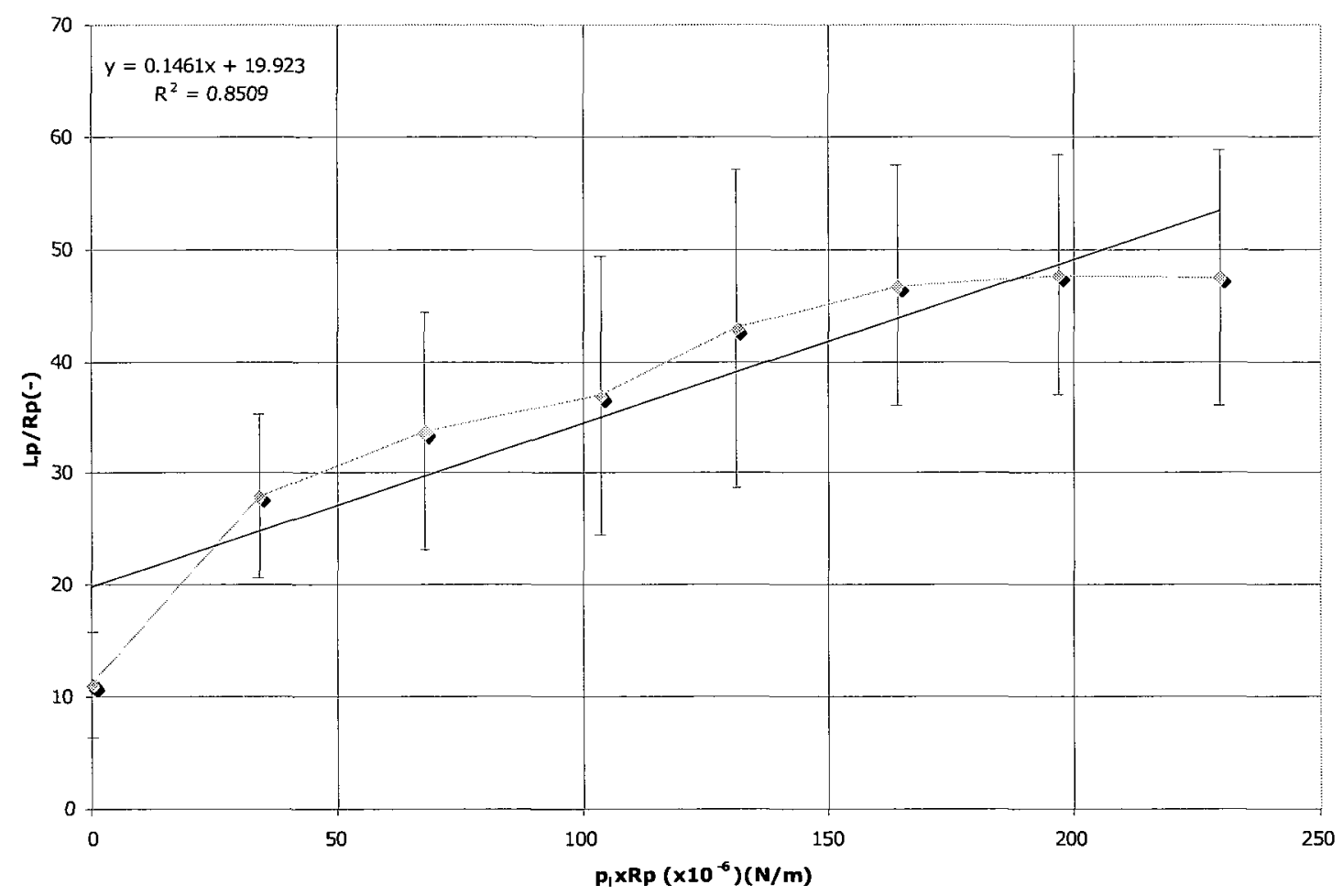

Figure 3.3: Average deformation behavior of murine platelets during micropipette aspiration. Linear regression is based on plotted average values. The standard deviations are a result of platelet and pipette radius variability, rather than measurement error.

\subsubsection{Discussion}

In addition to circumventing certain safety concerns surrounding equipment contamination with human blood samples, the murine alternative provided an opportunity to confirm the feasibility of micropipette aspiration for platelet deformation experiments, as robust platelet deformation data prove scarce. Namely, previous platelet deformation studies employing micropipette aspiration pursued alternative research interests, investigating platelet deformability responses in the presence of activation agonists (86), or in the absence of cytoskeletal structural components (66). Although useful to the current work to some extent, it was necessary to reintroduce the methodological fundamentals of this technique along with improved imaging software programs and tailored sample preparation procedures (Section 3.1 ), so as to confirm the overall deformation characteristics (shape transformations, material properties etc.) of the platelet. Specifically, Table 3.1 introduces the observed spherical shape of the unaspirated platelet fraction, identifying the ratio of the major and minor radii as 
approximately 1.0. As discussed in Sections 4.1.2 - 4.2, this geometric consistency reduces the complexity of future mathematical model developments. During deformation, the platelet membrane was also observed for evidence of viscoelastic behavior, however the membrane's progression into the pipette appeared to be dependent on applied pressure only. In terms of whole cell responses, preliminary evidence of platelet lysis was also observed, which may ultimately be used in subsequent analysis to determine the membrane strength. As quantified in terms of irreversible murine shape change from a discoid to spherical conformation, mechanically-induced platelet activation using this technique was not observed. This lack of mechanotransduction could potentially be impeded by the various sample processing steps (Section 3.1.2 - 3.1.3), which implemented a number of activation and aggregation antagonists to impede coagulation during the multiple sample washing steps. Alternatively, the limited range of applied negative pressure might also suggest that murine platelets possess certain hemostatic capabilities. Prevention of sample activation is also necessary to preserve membrane mechanical behavior, as previous aspiration studies by Burris et al. (86) demonstrated that activated platelet exhibit greater deformability.

Interestingly, the static deformation data outlined in Figure 3.2 and Figure 3.3 reveal membrane behavioural similarities between platelet species, as it is qualitatively similar to results obtained in studies by Haga et al. (11), Burris et al. (86) and White et al. (66) that used human platelet models. As with human platelets (66), this linear deformation behavior is observed to be independent of the local site of aspiration (i.e. platelet poles, equator), thereby supporting a simplistic material behavior (i.e. isotropic) assumption. It is also important to recognize that the accumulated platelet deformation data may vary in usefulness in the subsequent characterization of the platelet membrane. As emphasized in the theoretical development of subsequent chapters, platelet volume is assumed constant during micropipette aspiration. To adhere to this theoretical constraint, Figure 3.3 only presents the average deformation behavior of those murine platelets shown to maintain constant volume, so as to ultimately obtain an acceptable and representative deformation data set for subsequent mathematical model applications (Section 4.5.2). To assess the linearity of these data points, a trendline was fitted to this plot of average deformation behavior and generated a fairly reasonable prediction of applicable values, as the coefficient of determination $\left(R^{2}\right)$ is approximately .85 , which suggests that the correlation is strong (112). Estimating the 
Pearson correlation coefficient, $r$, also clarifies the extent of a linear relationship between the average values of $\frac{L_{p}}{R_{p}}$ and $p_{\mathrm{i}} \mathrm{xRp}$ outlined in Figure 3.2, as an estimate of approximately +.92 demonstrates a strong correlation (112). 


\section{Chapter 4}

\section{Mathematical Model}

Although the principles and methods required for the constitutive modeling of biological materials remains generally consistent, the development of each model requires an extensive and specific understanding of the biostructure in question. As such, the following Chapter provides a detailed description of platelet geometry, membrane material behavior and loading conditions necessary to model platelet membrane responses to micropipette aspiration. To accommodate this complex set of information, the Chapter also details those algorithms used for a solution in Matlab. Finally, the model's efficiency in predicting membrane deformation responses is presented.

\subsection{Geometric Assumptions}

\subsubsection{Geometry of Undeformed Platelet}

As mentioned, morphological studies characterizing an inactive, undeformed platelet have identified a discoid shape in circulation (13) with a geometry comparable to an oblate spheroid. An oblate spheroid is an ellipsoid that is rotationally symmetric about a polar axis (z-axis) with a shorter polar radius $\left(\mathrm{S}_{\mathrm{s}}\right)$ than the radius of the equatorial circle $\left(\mathrm{R}_{\mathrm{s}}\right)$ whose plane bisects this axis (i.e. $R_{\mathrm{s}}>S_{\mathrm{s}}$ ) (Figure 4.1). The coordinates $(X, Y, Z)$ of points on the surface of an idealized platelet are as follows (113),

$$
\begin{array}{lr}
X=R_{s} \sin \Phi \cos \Theta & \text { where } 0 \leq \Phi \leq \pi \\
Y=R_{s} \sin \Phi \sin \Theta & -\pi \leq \Theta \leq \pi \\
Z=S_{s}(1+\cos \Phi) . &
\end{array}
$$

The eccentricity, $e$, of an oblate spheroid is defined by,

$$
e \equiv \sqrt{1-\frac{S_{s}^{2}}{R_{s}^{2}}},
$$

where its surface area, $A_{0}$, is defined by,

$$
A_{o}=2 \pi R_{s}^{2}+\pi\left(\frac{S_{s}^{2}}{e}\right) \ln \left(\frac{1+e}{1-e}\right)
$$

The volume of an oblate spheroid is also a function of the polar $\left(S_{\mathrm{s}}\right)$ and equatorial $\left(R_{\mathrm{s}}\right)$ radii, 


$$
V_{o}=\frac{4}{3} \pi R_{s}^{2} S_{s}
$$

The platelet surface geometry in both the undeformed (uppercase notation) and deformed (lowercase notation) states can be treated as an axisymmetric shell. Essentially, the shape of an axisymmetric shell is generated by revolving a continuous curve or generator around the $Z$-axis (114). The distance along the generator (or meridian) is defined by $R=R(Z)$, and rotates about the $Z$-axis to form the middle surface of this shell of revolution (114). Relative to this surface, the thickness of the shell is uniform in both directions (114). The platelet is considered a thin axisymmetric shell, as the membrane thickness is significantly less than the minimum principal radius of curvature (Section 2.1) (114).

Further, any point along the middle surface of this shell can be described by an orthogonal coordinate system defined by $(N, S, \Theta)$. The $N$ coordinate is defined as the outward normal to the surface of the shell, the $S$ coordinate is the line tangent to the corresponding meridional line, while the $\Theta$ coordinate is the line tangent to a circle that passes through the point in question and is parallel to the $X Y$ plane (Figure 4.1) (114).



Figure 4.1: Undeformed platelet geometry. The platelet assumes an oblate spheroid geometry when inactive in circulation. An illustration of a specific element lying on the axisymmetric surface of the bottom oblate hemispheroid is defined by points $\mathrm{A}$ to $\mathrm{D}$ in the inactive (undeformed) shape and correspond to $\mathrm{A}(R, \Theta, Z), \mathrm{B}$ $(R+d R, \Theta, Z+d Z), \mathrm{C}(R, \Theta+d \Theta, Z), \mathrm{D}(R+d R, \Theta+d \Theta, Z+d Z)$. 
In the undeformed geometry, the differential positions along the contour of the axisymmetric shell are as follows, with

$$
\begin{aligned}
R^{2} & =X^{2}+Y^{2}, \text { and therefore } \\
R & =R_{s} \sin \Phi \\
d R & =R_{s} \cos \Phi d \Phi, \\
d Z & =-S_{s} \sin \Phi d \Phi, \\
d S & =\sqrt{d R^{2}+d Z^{2}}=\sqrt{R_{s}^{2} \cos ^{2} \Phi+S_{s}^{2} \sin ^{2} \Phi} d \Phi .
\end{aligned}
$$

Defining the undeformed geometry of the platelet will prove useful in future model developments (Section 4.4.1) that impose geometric constraints on platelet deformation behavior.

\subsubsection{Geometry of Deformed Platelet during Micropipette Aspiration}

To better characterize the geometry and associated equations that dominate axisymmetric shell calculations, with specific relevance to the deformed state, one must include a number of additional variables (Figure 4.2). The angle $\phi$ defines the angle between the shell surface normal and the axis of rotation (z-axis). Along the $n$ direction, the distance $r_{\theta}$ is defined by the midsurface of the shell and the $z$-axis, and is recognized to be a principle curvature at the point $(s, \theta)$ along the shell surface (114). The distance $r_{\mathrm{n}}$ defines a second principal curvature of the shell surface that lies along the $n$ direction, and is the local arc in the meridional plane (114).

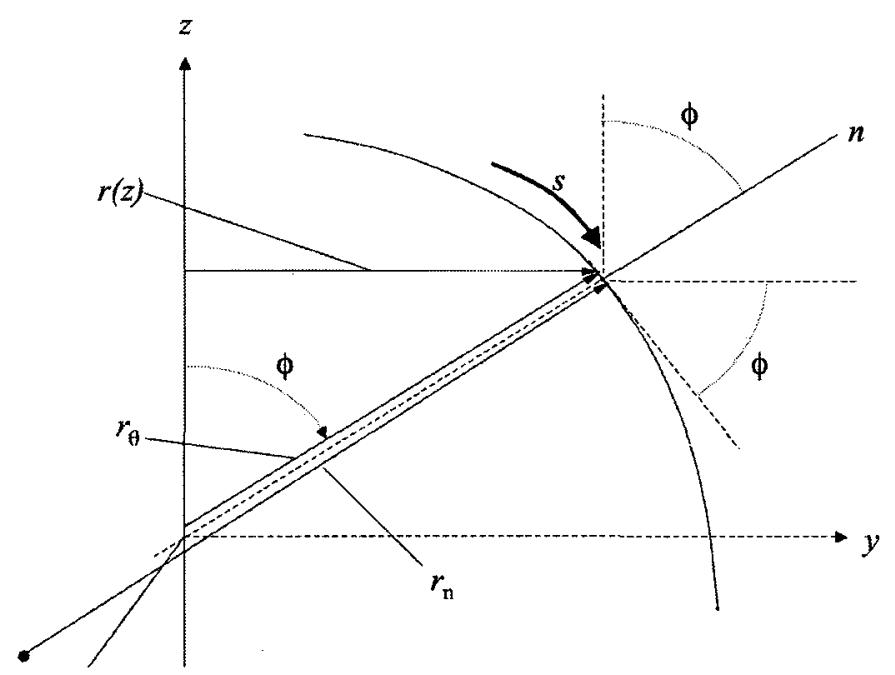

Figure 4.2: Shell surface geometry for a deformed platelet. [Adapted from (114)]. 
In the context of the current research, a platelet deformed by micropipette aspiration with an axisymmetric shell geometry is subjected to variable magnitudes of distension pressure, which is location-dependent and defined by three major 'zones' (Figure 4.3). During micropipette aspiration, the platelet geometry is described in terms of the unaspirated, spherical shell (zone 1), the aspirated cylindrical shell (zone 2) and the aspirated hemispherical shell (zone 3).

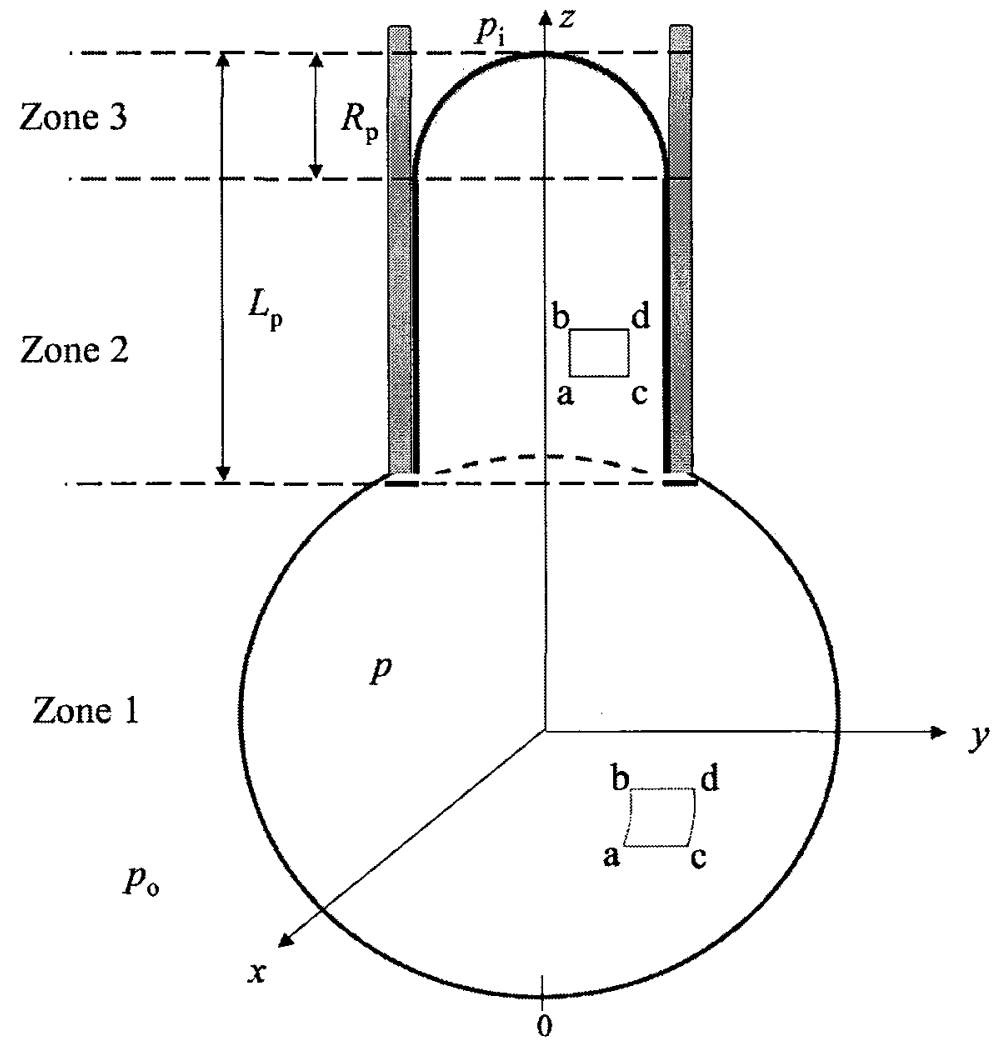

Figure 4.3: Proposed contour for an aspirated platelet. Contact between the platelet membrane and the micropipette during aspiration assumed to occur along the front face and inner wall of the pipette. Zones 1 to 3 define the spherical shape, the circular cylindrical shape, and the hemispherical shape of the deformed platelet, respectively. The aspirated platelet length $\left(L_{\mathrm{p}}\right)$ and the inner radius of the pipette $\left(R_{\mathrm{p}}\right)$ are shown. Atmospheric pressure $\left(p_{0}\right)$, intracellular pressure $(p)$ and aspiration pressure $\left(p_{\mathrm{i}}\right)$ contribute to distension pressure.

For all zones (See Appendix B), the relationship between the radius $r=r(z)$ which generates a meridional line and $r_{\theta}$ is defined by (114),

$$
r=r_{\theta} \sin \phi \text {. }
$$

As introduced in Section 3.2.2, the present development assumes that the unaspirated portion of a platelet (zone 1) maintains a spherical geometry, where a sphere of radius, $r_{\mathrm{s}}$, and the principal curvatures $r_{\theta}$ and $r_{\mathrm{n}}$ are presumed equal $\left(r_{\mathrm{s}}=r_{\theta}=r_{\mathrm{n}}\right)(101,114)$. Further, the 
constraints of the glass micropipette with uniform internal radius $\left(R_{\mathrm{p}}\right)$ are assumed to limit membrane behavior (zone $2, r_{\theta}=R_{\mathrm{p}}, r_{\mathrm{n}}=\infty$; zone $3, r_{\theta}=r_{\mathrm{n}}=R_{\mathrm{p}}$ ). The radii can therefore be expressed as such,

$$
\begin{array}{lll}
\text { Zone 1: } & r=r_{s} \sin \phi & \phi_{s} \leq \phi \leq \pi \\
\text { Zone 2: } & r=R_{p} & \phi=\frac{\pi}{2} \\
\text { Zone 3: } & r=R_{p} \sin \phi & 0 \leq \phi \leq \frac{\pi}{2}
\end{array}
$$

Similarly, the length component parallel to the $z$-axis varies with each zone, with notable contributions by the aspirated platelet length $\left(L_{\mathrm{p}}\right)$ in zones 2 and 3 .

$$
\begin{array}{lll}
\text { Zone } 1: & z & =r_{s}(1+\cos \phi) \\
\text { Zone 2: } & & z=r_{s}\left(1+\cos \phi_{s}\right) \leq z \leq r_{s}\left(1+\cos \phi_{s}\right)+L_{p}-R_{p} \\
\text { Zone 3: } & z & =r_{s}\left(1+\cos \phi_{s}\right)+L_{p}-\mathrm{R}_{\mathrm{p}}(1-\cos \phi)
\end{array}
$$

The boundary angle $\phi_{\mathrm{s}}$ corresponds to angle between the shell surface normal and the axis of rotation at the transition point between zone 1 (spherical shell) and zone 2 (cylindrical shell). This value is constant, and is a function of inner pipette radius $\left(R_{\mathrm{p}}\right)$ and sphere radius $\left(r_{\mathrm{s}}\right)$,

$$
\phi_{s}=\arcsin \left(\frac{R_{p}}{r_{s}}\right) \text {. }
$$

Variable position along each axisymmetric shell contour is described by differential lengths $(d r, d z, d s)$ that are as follows,

$$
\begin{array}{ll}
\text { Zone 1 }(83,114): & d r=d s \cos \phi ; d z=-d s \sin \phi ; d s=r_{n} d \phi \\
\text { Zone 2: } & d r=0 ; d z=d s ; d \phi=0 \\
\text { Zone 3: } & d r=d s \cos \phi ; d \phi=-d s \sin \phi ; d s=R_{p} d \phi
\end{array}
$$

These relationships will ultimately contribute to the development of equilibrium equations (Section 4.2) for the platelet membrane acting as an axisymmetric shell (114).

As mentioned in Section 2.5.2, the deformed platelet must also be described in terms of extension ratios, that define circumferential stretch $\left(\lambda_{\theta}\right)$ and longitudinal stretch $\left(\lambda_{s}\right)$. These stretch magnitudes can be identified by relating the defining points (A to D) of an element lying on the undeformed axisymmetric surface (Figure 4.1) to the deformed alternative (a to d) (Figure 4.3). This elemental relationship can also be defined in curvilinear coordinates for visual clarity purposes, as illustrated in Appendix C. Evidently, membrane 
stretch will change across zones due to variable constraints (i.e. glass micropipette), and are therefore represented as such:

Zone 1 (Sphere of radius $r_{s}$ ), where $\Phi_{s} \leq \Phi \leq 0$ and $\Phi_{s}=\arcsin \left(\frac{R_{p}}{r_{s}}\right)$ :

$$
\begin{aligned}
& \lambda_{\theta}=\frac{a c}{A C}=\frac{r d \theta}{R d \Theta}=\frac{r}{R_{s} \sin \Phi}=\frac{r_{s} \sin \phi}{R_{s} \sin \Phi}, \\
& \lambda_{s}=\frac{a b}{A B}=\frac{d s}{d S}=\left(\frac{r_{s}}{\sqrt{R_{s}^{2} \cos ^{2} \Phi+S_{s}^{2} \sin ^{2} \Phi}}\right) \frac{d \phi}{d \Phi}
\end{aligned}
$$

Zone 2 (Cylinder of radius $R_{p}$ ), where $\Phi_{1} \leq \Phi \leq \Phi_{s}$ :

$$
\begin{aligned}
& \lambda_{\theta}=\frac{a c}{A C}=\frac{r d \theta}{R d \Theta}=\frac{r}{R_{s} \sin \Phi}=\frac{R_{p}}{R_{s} \sin \Phi}, \\
& \lambda_{s}=\frac{a b}{A B}=\frac{d s}{d S}=\left(\frac{1}{\sqrt{R_{s}^{2} \cos ^{2} \Phi+S_{s}^{2} \sin ^{2} \Phi}}\right) \frac{d z}{d \Phi}
\end{aligned}
$$

Zone 3 (Hemisphere of radius Rp), where $0 \leq \Phi \leq \Phi_{t}$ and $\Phi_{t}=\frac{\pi}{2}$ :

$$
\begin{aligned}
& \lambda_{\theta}=\frac{a c}{A C}=\frac{r d \theta}{R d \Theta}=\frac{r}{R_{s} \sin \Phi}=\frac{R_{p} \sin \phi}{R_{s} \sin \Phi} \\
& \lambda_{s}=\frac{a b}{A B}=\frac{d s}{d S}=\left(\frac{R_{p}}{\sqrt{R_{s}{ }^{2} \cos ^{2} \Phi+S_{s}^{2} \sin ^{2} \Phi}}\right) \frac{d \phi}{d \Phi} .
\end{aligned}
$$

Finally, it is also important to describe the behavior of the entire deformed platelet in terms of the final surface area $(A)$ and volume $(V)$, which are calculated by taking the sum of the three zones:

$$
\begin{aligned}
A & =A_{\text {sphere }}-A_{\text {spherical cap }}(116)+A_{\text {cylinder }}+A_{\text {hemispherical cap }} \\
& =4 \pi r_{s}^{2}-2 \pi r_{s}\left(r_{s}-\sqrt{r_{s}^{2}-R_{p}^{2}}\right)+2 \pi\left(L_{p}-R_{p}\right) R_{p}+2 \pi R_{p}{ }^{2} \\
V & =V_{\text {sphere }}-V_{\text {spherical cap }}(116)+V_{\text {cylinder }}+V_{\text {hemispherical cap }} \\
& =\frac{4}{3} \pi r_{s}^{3}-\frac{\pi}{3}\left(r_{s}-\sqrt{r_{s}{ }^{2}-R_{p}^{2}}\right)^{2}\left(2 r_{s}+\sqrt{r_{s}{ }^{2}-R_{p}{ }^{2}}\right)+\pi\left(L_{p}-R_{p}\right) R_{p}{ }^{2}+\frac{2}{3} \pi R_{p}^{3}
\end{aligned}
$$

Along with the undeformed calculations of platelet surface area $\left(A_{\mathrm{o}}\right)$ and volume $\left(V_{\mathrm{o}}\right)$, determining these deformed values may confirm useful platelet characteristics (i.e. incompressibility) that could reduce the complexity of the mathematical model (98). Specifically, the theoretical development for the platelet membrane is reduced to two dimensions by assuming the cell volume is conserved (i.e. $V_{\mathrm{o}}=V$ ) following deformation. 


\subsection{Equilibrium Equations for a Platelet using Micropipette Aspiration}

As adopted in previous erythrocyte membrane models (Section 2.5.3), the current mathematical model for the platelet membrane employs the equations of mechanical equilibrium for a thin axisymmetric shell (114), where only loading that primarily produce membrane forces are considered. Based on conclusions made by Fung and Tong (101) using erythrocytes, the dominant stresses in a thin shell are primarily membrane stresses, with negligible bending or radial stress contributions. For erythrocytes and platelets alike, the thickness of the membrane is such that there is not an appreciable distance between the extracellular and intracellular membrane surface: a morphological property that impedes significant bending stresses (101). Fung and Tong (101) also concluded that for thin shells, which are defined in cylindrical coordinates, shear stresses $T_{\mathrm{s} \theta}$ and $T_{\theta \mathrm{\theta}}$ are considered equal and negligible when symmetrically loading a surface of revolution. To justify this assumption, one must consider the remaining principal stresses (circumferential/hoop stress and longitudinal/axial stress) in the membrane, which are constant over the axisymmetric shell geometry. Along these orthogonal planes, then, the shear stress is zero (115). It is also accepted that if the Gaussian curvature, defined as the product of the two principle curvatures at any point $(s, \theta)$, is positive over the entirety of the platelet, then the absence of shear stress holds true (98).

Once static equilibrium is reached following micropipette aspiration at a uniform magnitude of negative pressure, the resultant internal forces are defined by the circumferential tension, $T_{\mathrm{\theta}}$, acting in the $\theta$ direction and the meridional tension, $T_{\mathrm{s}}$, acting in the $s$ direction (114). Surface loading of thin axisymmetric shells can include distension pressure values $p_{\mathrm{n}}, p_{\mathrm{s}}$, and $p_{\mathrm{\theta}}$, which are continuous along the membrane at any point $(s, \theta)$ (114). Ultimately, however, only the distension pressure that is normal to the membrane surface $\left(p_{\mathrm{n}}\right)$ is considered (i.e. $p_{\mathrm{\theta}}=p_{\mathrm{s}}=0$ ) in subsequent micropipette aspiration applications using the following equilibrium equations (114): 


$$
\begin{aligned}
\frac{T_{\theta}}{r_{\theta}}+\frac{T_{s}}{r_{n}} & =p_{n}, \\
\frac{\partial \mathrm{T}_{\theta}}{\partial \theta} & =0, \\
\frac{1}{r_{\mathrm{n}}}\left(\frac{\partial T_{s}}{\partial \phi}\right)+\frac{1}{r}\left(T_{s}-T_{\theta}\right) \cos \phi+p_{s} & =0 .
\end{aligned}
$$

For simplicity, these equilibrium equations can be expressed as follows (See Appendix D) (98),

$$
\begin{aligned}
& T_{s}=\left(\frac{p_{n}}{2}\right) r_{\theta}, \\
& T_{\theta}=p_{n} r_{\theta}\left(1-\frac{r_{\theta}}{2 r_{n}}\right) .
\end{aligned}
$$

These equilibrium equations will vary across the deformed platelet, and are dependent on zone geometry as well as the variable contributions of atmospheric pressure $\left(p_{\mathrm{o}}\right)$, uniform intracellular pressure $(p)$ and aspiration pressure $\left(p_{\mathrm{i}}\right)$. Using superscripts to refer to zones, Zone 1 (Sphere of radius $r_{s}$ ):

$$
T_{s}^{1}=\left(\frac{p-p_{o}}{2}\right) r_{s}=T_{\theta}^{1}
$$

Zone 2 (Cylinder of radius $R_{\mathrm{p}}$ ):

$$
\begin{aligned}
& T_{s}^{2}=\left(\frac{p-p_{o}}{2}\right) r_{s}=\left(\frac{p-p_{i}}{2}\right) R_{p}=T_{s}^{1}=T_{s}^{3}, \\
& \text { therefore } \mathrm{p}=\frac{\mathrm{p}_{0} r_{s}-p_{i} R_{p}}{r_{s}-R_{p}} \\
& \mathrm{~T}_{\theta}^{2}=p R_{p}
\end{aligned}
$$

Zone 3 (Hemisphere of radius $R_{p}$ ):

$$
\mathrm{T}_{\mathrm{s}}^{3}=\left(\frac{p-p_{i}}{2}\right) R_{p}=T_{\theta}^{3} .
$$

Of interest, the sole contribution in zone 2 to distension pressure is the intracellular pressure $(p)$, as the confines of the glass micropipette impede atmospheric pressure contributions. As this variable is not directly quantifiable in the context of the current work, intracellular pressure is ultimately identified by assuming that the membrane behaves homogeneously (i.e. $T_{\mathrm{s}}{ }^{1}=T_{\mathrm{s}}{ }^{2}=T_{\mathrm{s}}{ }^{3}$ ). Further, the circumferential tension in this zone $\left(T_{\theta}{ }^{2}\right)$ is also limited by 
the micropipette, thereby impeding its usefulness in characterizing the material properties of the platelet membrane.

\subsection{Material Model}

Complementing the theoretical developments of both erythrocyte membrane models (Section 2.5.3), the current constitutive model for the platelet membrane also postulates a strain energy function, $W$, implementing an invariant defined by Skalak et al. (94). The novel logarithmic strain energy function incorporates a univariate $\left(I_{2}\right)$ polynomial, $Q$, and is developed as follows,

$$
W=-\frac{c_{1}}{2} \ln (1-Q) \quad \text { with } Q=\mathrm{c}_{2} I_{2}^{n_{2}}+c_{3} I_{2} \text { and } I_{2}=\lambda_{1}^{2} \lambda_{2}^{2}-1
$$

The hyperelastic behavior of the membrane is described by this strain energy function and is ultimately used to derive a stress-strain relationship (See Appendix E) which automatically accounts for the spherical portion (i.e. $T_{\mathrm{s}}=T_{\theta}$ ) of the deformed platelet, since $W=W\left(I_{2}\right)$ is upheld ${ }^{3}$ :

$$
T_{s}=T_{\theta}=2 \lambda_{s} \lambda_{\theta} \frac{\partial W}{\partial I_{2}}
$$

Clearly, with the condition that the deformed platelet assumes an axisymmetric shape, the principal axes of stress and strain follow a circumferential and longitudinal direction, respectively. Unlike the developments by Evans (98) and Skalak et al. (94), however, the preservation of areal strain is not prerequisite, as membrane incompressibility is not directly considered for the platelet membrane, thereby avoiding those model limitations (Section 2.5.3) inherent to both erythrocyte models. Finally, the current work also considers the residual tension $\left(T_{0}\right)$ in the platelet membrane, such that:

$$
T_{s}=T_{\theta}=2 \lambda_{s} \lambda_{\theta} \frac{\partial W}{\partial I_{2}}+T_{o}
$$

Numerous investigations mapping the stress characteristics of cell membranes have revealed this inherent load-free tension, with quantified cases of isometric tension sustained by the supporting cytoskeletal components of the cell (117). If one ignores the presence of prestress in the membrane, the global mechanical equilibrium of the platelet membrane would be

\footnotetext{
${ }^{3}$ Note that membrane stresses and extension ratios in principal directions are directly analogous to the conventions used by Evans (98), Skalak et al. (94) and Humphrey et al. (97).
} 
inaccurate. Significant to current definitions of platelet geometry in the undeformed and deformed shapes, residual stress is also recognized to be a critical determinant of cell shape stability, as diminished intracellular tension increases cell flexibility (117). Further, mechanotransduction pathways are also partially controlled by the structural integrity of the cell, as governed by the global mechanical stability (including prestress) of the cell (117). Evidently, by incorporating prestress in the mathematical description of membrane tension, one can improve the overall accuracy of the constitutive model in predicting membrane mechanical responses. As such, the tension generated in the membrane is described in terms of the mechanical equilibrium equations (Section 4.2), the above constitutive descriptions, and prestress.

\subsection{Problem Formulation and Method of Solution}

The development of a mathematical model that accurately predicts the mechanical behavior of the platelet membrane in response to an applied load requires a multifaceted and interdependent approach. To date, constitutive models for the platelet membrane are nonexistent, as preliminary efforts to employ membrane models of different cell types (i.e. erythrocytes) prove flawed due to variability in geometry and global deformation responses.

To parallel those general constitutive modeling principles and methods outlined in Section 2.5 , the present approach to platelet membrane modeling is one which integrates the salient features of this method, but characterizes the specific mechanism of cell deformation in terms of platelet membrane responses to micropipette aspiration. The main objective of this work is to ultimately propose constitutive equations and material constants for the human and mouse platelet membranes that accurately describe the respective membrane responses to a specific form of mechanical manipulation. The preliminary modeling steps that extensively define the platelet's shape transformation and material model (i.e. constitutive equations, prestress) are applicable to both platelet species, as Section 2.1.3 emphasizes their structural and functional homology. Once the undeformed geometry and stress response characteristics to general loading conditions are defined, the model also incorporates a resultant, deformed geometry, along with a comprehensive description of those membrane stresses generated in response to micropipette aspiration. Initially, the geometric constraints on the aspirated platelet are predominantly hypothetical, based solely on limited aspiration 
images of human platelets (11). Further, the complexity of the model is reduced to a twodimensional evaluation of membrane deformation responses, assuming that platelet volume is stable during the aspiration process. As discussed later in Section 4.5, these hypotheses are validated from micropipette aspiration performed on a murine platelet model.

For simplicity, the deformed platelet is segmented into three well-defined and quantifiable geometries (Section 4.1.2), and is proposed to maintain a constant volume over a range of deformation described in terms of extension ratios $\left(\lambda_{s}, \lambda_{\theta}\right)$. The model also defines a set of quantifiable variables consistent between the undeformed and deformed geometries, and adjusts the magnitude of a specific variable (i.e. ' $r_{\mathrm{s}}$ ') to satisfy the volume constraint by complying with inputted experimental measurements of aspirated platelet fractions (i.e. ' $L_{\mathrm{p}}$ '). Further, solutions for the extension ratio ' $\lambda_{\mathrm{s}}$ ' in zone 2 (cylindrical shape) are only deemed acceptable when platelet volume is conserved, which is maintained when the length of this platelet fraction is indeed $L_{\mathrm{p}}-R_{\mathrm{p}}$ (Figure 4.3). To satisfy equilibrium equations and this final constraint, the model utilizes the Levenberg-Marquardt method, which is an optimization method that identifies material constants $\left(\mathrm{c}_{\mathrm{i}}\right)$ that minimizes the error on the deformed length of zone 2 .

\subsubsection{Equations to Solve}

The mechanism that ultimately defines independent and optimized sets of membrane material constants for the human and murine platelet membrane is common to both, but differs slightly in experimental descriptions of membrane deformability, which will be discussed in a later Section 4.5. Beginning with geometry and size definitions, the initial platelet volume for the human platelet is based on an average value found in literature (11, 8 ), while the initial murine platelet volume was calculated using experimental length measurements $\left(R_{\mathrm{s}}, S_{\mathrm{s}}\right)$ of inactive platelets collected in the current study. In addition to this information, deformation data such as the pipette radius $\left(R_{\mathrm{p}}\right)$, the range of aspiration pressures $\left(p_{\mathrm{i}}\right)$ and the resultant aspirated platelet lengths $\left(L_{\mathrm{p}}\right)$ prove useful in subsequent computations in the deformed state.

Specifically, the experimentally observed $L_{\mathrm{p}}$ and $R_{\mathrm{p}}$ values are useful in the first significant calculation, which identifies the radius $\left(r_{\mathrm{s}}\right)$ of the deformed spherical protrusion (zone 1) that maintains cell volume during the shape transformation: 


$$
0=V_{o}-\left(\frac{4}{3} \pi r_{s}^{3}-\frac{\pi}{3}\left(r_{s}-\sqrt{r_{s}^{2}-R_{p}^{2}}\right)^{2}\left(2 r_{s}+\sqrt{r_{s}^{2}-R_{p}^{2}}\right)+\pi\left(L_{p}-R_{p}\right) R_{p}^{2}+\frac{2}{3} \pi R_{p}^{3}\right) .
$$

The $r_{\mathrm{s}}$ value is then used to calculate the internal pressure $(p)$ of the platelet using the following calculation:

$$
p=\frac{p_{o} r_{s}-p_{i} R_{p}}{r_{s}-R_{p}}
$$

Subsequent evaluations of membrane tension are organized into zones, as equations vary between these regions (Section 4.1.2). For zone 1 and 3, the membrane equilibrium in these regions following aspiration is similarly described by fully implicit differential equations of the form $f\left(\Phi, \phi, \frac{d \phi}{d \Phi}\right)=0$, while zone 2 is alternatively described. Specifically, membrane equilibrium is represented as:

Zone 1: $\quad\left(p-p_{0}\right) r_{s}-2 c_{1} \lambda_{s} \lambda_{\theta}\left(\frac{Q^{\prime}}{1-Q}\right)-2 T_{o}=0$

Zone 2: $\quad\left(p-p_{\mathrm{i}}\right) R_{p}-2 c_{1} \lambda_{s} \lambda_{\theta}\left(\frac{Q^{\prime}}{1-Q}\right)-2 T_{o}=0 \quad$ where $Q^{\prime}=\frac{\partial Q}{\partial I_{2}}$

Zone 3: $\quad\left(p-p_{\mathrm{i}}\right) R_{p}-2 c_{1} \lambda_{s} \lambda_{\theta}\left(\frac{Q^{\prime}}{1-Q}\right)-2 T_{o}=0$

Evidently, these descriptions of membrane equilibrium follow similar equations, but differ in the complexity of each evaluation, which is dependent on the method of extension ratio (i.e. $\lambda_{s}, \lambda_{\theta}$ ) identification. Since zone 1 and 3 conform to a spherical geometry and represent opposite poles of the deformed macrostructure, one can impose specific boundary conditions at the poles (i.e. $\Phi=\phi$ ) and assume that the magnitude of membrane extension is identical in the radial and circumferential directions (i.e. $\lambda_{s}=\lambda_{\theta}$ ). One can then solve numerically for the unknown variables $\left(\frac{d \phi}{d \Phi}\right)_{\pi}$ and $\left(\frac{d \phi}{d \Phi}\right)_{0}$, which can be alternatively expressed as $\kappa_{1}$ and $\kappa_{3}$, respectively (See Appendix F). Deciphering the membrane stresses in zone 2, however, requires an alternative approach to solving $\lambda_{s}$ and $\lambda_{\theta}$ that cannot be simplified by imposing boundary conditions. Notably, the membrane of this aspirated platelet fraction maintains a cylindrical shape whose extension is governed by the radius of the pipette $\left(R_{p}\right)$ and the differential length along the axis of symmetry $(d z)$. Thus, the solution for $\lambda_{\theta}$ is easily defined, but requires a novel approach for the evaluation of $\lambda_{s}$, since: 


$$
\lambda_{\theta}=\frac{R_{p}}{R_{s} \sin \Phi} ; \lambda_{\mathrm{s}}=\left(\frac{1}{\sqrt{R_{s}^{2} \cos ^{2} \Phi+S_{s}^{2} \sin ^{2} \Phi}}\right) \frac{d z}{d \Phi} \quad \text { where } \Phi_{t} \leq \Phi \leq \Phi_{s} .
$$

Since one cannot directly evaluate $\frac{d z}{d \Phi}$, acceptable $\lambda_{s}$ values that satisfy the conservation of volume assumption are identified using the Levenberg-Marquardt (L-M) method. Essentially, $\lambda_{s}$ values are acceptable when the numerical error $(\varepsilon)$ is minimal between the longitudinal deformation in zone 2 and the experimental length $\left(L_{\mathrm{p}}-R_{\mathrm{p}}\right)$ :

$$
\left|\int_{\Phi_{t}}^{\Phi_{s}} \lambda_{s} \sqrt{R_{s}^{2} \cos ^{2} \Phi+S_{s}^{2} \sin ^{2} \Phi} d \Phi-L_{p}-R_{p}\right|<\varepsilon .
$$

To find this minimum error, the material constants $\left(\mathrm{c}_{\mathrm{i}}\right)$ are iteratively adjusted and ultimately defined using this optimization method.

\subsubsection{Algorithms}

To achieve the aforementioned objectives, the mathematical model first introduces the experimental deformation data (i.e. aspiration pressure, $L_{\mathrm{p}}$ values) and also defines a set of variables necessary in later evaluations of the deformed platelet. Once a representative geometric foundation is defined, the model sequentially reduces acceptable solutions for the membrane material constants by implementing the Levenberg-Marquardt (L-M) method. This iterative algorithm provides a robust and definitive approach to solving non-linear least square problems, and is considered a hybrid alternative to the Gauss-Newton (G-N) and steepest descent optimization methods. Although the G-N method minimizes the number of iteration requirements, its convergence efficiency is only optimal when the initial solution is close to the desired minimum solution. Interestingly, when this proximity condition is met, the Levenberg-Marquardt method behaves similar to the Gauss-Newton method. Conversely, by behaving like the steepest descent method the L-M method can also accommodate situations beginning with an initially large range of solutions at the cost of computation time. Focusing on the current problem then, this complex algorithm requires a number of highly interdependent calculations, and thus demands a level of organization described in the following flowchart (Figure 4.4, a-b): 
(a)

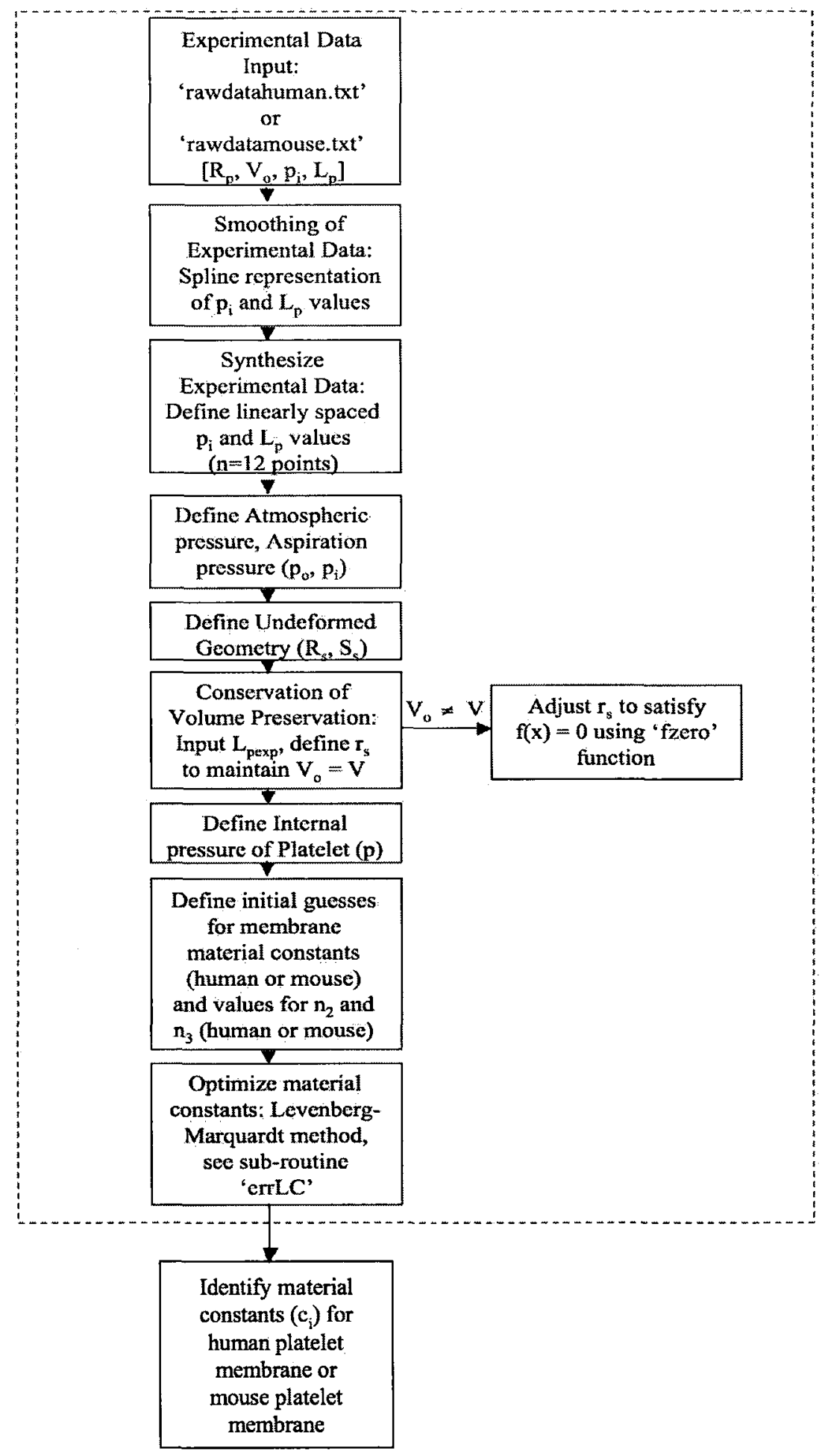


(b)



Figure 4.4: Iterative algorithm to identify membrane material constants. Schematic of the MATLAB program '1-Material Constants Identification' details of (a) M-file: 'main' and (b) M-file: 'errLC'. 
Evidently, the final identification of platelet membrane material constants requires the conjunctive efforts of the M-file 'main', which synthesizes and defines salient variables with global applications, and the function 'errLC', which characterizes the membrane deformation responses in each geometrically independent zone of the aspirated platelet. Most notably, the m-file 'main' also introduces the Levenberg-Marquardt method. Essentially, this optimization method solves problems of the form $\sum\left\{\operatorname{errLC}(\mathrm{x})^{2}\right\}$, by finding a minimum solution to the sum of squares of the functions defined in 'errLC'. To begin iterating, initial species-sensitive guesses for material constants $\left(c_{i}, i=1-4\right.$; $c_{4}$ defines prestress $\left.\left(T_{0}\right)\right)$ are inputted along with constants $n_{2}$ and $n_{3}$, which may generate a set of material constants that fail to produce a minimum numerical error $(\varepsilon)$ (Section 4.4.1). With continued iterations narrowing the range of acceptable material constants, the program ultimately yields a vector of optimized material constants that conserve platelet and produce a minimum error $(\varepsilon)$ between the length parameters.

\subsubsection{MATLAB Implementation}

To execute the computation demands of the algorithm above, MATLAB (version 7.4) offers an effective set of functions that coordinately work to produce a set of optimized platelet membrane material constants (See Appendix G). Although mentioned briefly, it is necessary to detail the features of some fundamental functions, as they preserve the integrity of the model by maintaining certain theoretical assumptions (Section 4.1.2) that pertain to platelet deformation behavior.

As the mathematical model seeks to accurately predict the membrane deformation behavior of the platelet by identifying specific material constants, a set of experimental data detailing the deformation response of the platelet membrane to micropipette aspiration is first introduced in the M-file 'main'. This deformation data characterizes the sequential advance of the platelet membrane into a micropipette, as controlled by the magnitude of applied negative pressure. The undeformed dimensions of the platelet are also indirectly introduced, and are related by a generally accepted shaperatio $\left(\frac{S_{s}}{R_{s}}=0.15-0.25\right)$ for human platelets (14). To attain a more comprehensive description of membrane behavior, cubic spline 
interpolation of the experimental data set is executed using the 'spline' function. This function offers an alternative to single polynomial interpolation, as it provides a more representative description of experimental data by fitting several continuous polynomials to the data set. Following this data curve fitting, a set of linearly spaced data is identified and synthesized using the functions 'linspace' and 'ppval', respectively. The synthesized data set describing the length of the aspirated platelet $\left(L_{\mathrm{p}}\right)$ is then used to identify the spherical radius $\left(r_{\mathrm{s}}\right)$ of the deformed platelet that preserves volume, as isolated by the 'fzero' function. By first establishing a function of the form $f=V_{o}-V$ and an initial input $R_{\mathrm{s}}$, 'fzero' solves for $r_{\mathrm{s}}$ such that $f=0$. An acceptable value of $r_{\mathrm{s}}$ is ultimately identified by fzero at its value where the function $f$ changes sign, which indicates that volume is indeed preserved (i.e. $V_{\mathrm{o}}=V$ ). Following these evaluations, MATLAB can then implement the Levenberg-Marquardt method, whose aforementioned optimization properties are carried out by the 'Isqnonlin' function that evaluates 'errLC'. In terms of 'Isqnonlin' function specifications, lower and upper limits to the solution are set as undefined, as no bounds exist for the minimum solution. The Levenberg-Marquardt method also requires a number of predetermined option settings, including 'MaxFunEvals' set to $1 \mathrm{e} 100$, that ultimately allows an infinite number of function evaluations, 'MaxIter' set to $5 \mathrm{e} 3$, which allows a maximum of 5000 iterations before termination of the estimation. Both 'TolFun' and 'TolX' are also optimization parameters that influence the Levenberg-Marquardt process, and are set at 1e-5 and 1e-8 to indicate the termination tolerance values on the function value and parameter estimates respectively.

To evaluate the implicit differential equations outlined in the M-file 'errLC' and Section 4.4.1, it is necessary to enforce specific boundary conditions. To achieve this end, the angle $\phi$ (angle between the shell surface normal and the axis of rotation) and its undeformed geometry equivalent, $\Phi$, were presumed equal at the poles, such that one can also equate extension ratios $\lambda_{s}$ and $\lambda_{\theta}$. Although invaluable to the solutions of $\left(\frac{d \phi}{d \Phi}\right)_{\pi}$ and $\left(\frac{d \phi}{d \Phi}\right)_{0}$, one must make a clear distinction between these angles $(\Phi$ and $\phi)$ in MATLAB, so as to prevent a numerical crash. As such, one must distinguish each variable slightly away from the poles to avoid computational problems, differing only by a magnitude of epsilon $(\varepsilon)$. 
Zone 1: At approximately $\pi$,

$$
\begin{aligned}
\left(\frac{d \phi}{d \Phi}\right)_{\pi} & =\kappa_{1} \\
(d \phi)_{\pi} & =\kappa_{1}(d \Phi)_{\pi} \\
\phi & =\kappa_{1} \Phi+m_{1} .
\end{aligned}
$$

By imposing the boundary condition that $\Phi=\phi=\pi$, the constant $m_{1}$ is clearly defined $\left(m_{1}=\left(1-\kappa_{1}\right) \pi\right)$ and $\phi$ can be expressed as follows,

$$
\phi=\kappa_{1} \Phi+\left(1-\kappa_{1}\right) \pi, \quad \text { and } \quad \text { when } \Phi=\pi-\varepsilon, \phi=\pi-\kappa_{1} \varepsilon .
$$

Zone 3: At approximately 0,

$$
\begin{aligned}
\left(\frac{d \phi}{d \Phi}\right)_{0} & =\kappa_{3} \\
(d \phi)_{0} & =\kappa_{3}(d \Phi)_{0} \\
\phi & =\kappa_{3} \Phi+m_{3} .
\end{aligned}
$$

Conversely, by imposing the boundary condition that $\Phi=\phi=0$, the constant $m_{3}$ is clearly defined $\left(m_{3}=0\right)$ and $\phi$ can be expressed as follows,

$$
\phi=\kappa_{3} \Phi, \quad \text { and finally, } \quad \text { when } \Phi=\varepsilon, \phi=\kappa_{3} \varepsilon .
$$

After imposing the specific boundary conditions, defining the unknown magnitude of $\frac{d \phi}{d \Phi}$ at each pole is facilitated by implementing the 'fsolve' function in MATLAB. This function solves a system of nonlinear equations of several variables of the form $f(x)=0$ which are defined independently in fun 1 and fun 3 by first inputting an arbitrary scalar value (x0) and returning solutions for $\left(\frac{d \phi}{d \Phi}\right)_{\pi}$ and $\left(\frac{d \phi}{d \Phi}\right)_{0}$, respectively. As is applicable to other functions, the performance of 'fsolve' can be improved by replacing the default optimization parameters defined by the 'optimset' function. In this function, both 'Tolfun' and 'Tolx' are set to $1 \mathrm{e}-20$ and $1 \mathrm{e}-12$, and indicate the termination tolerance on the function value and parameter estimates respectively.

Following the identification of ' $\frac{d \phi}{d \Phi}$, at the poles, one must then define a set of new data using the function 'ode15i', by integrating a system of differential equations (ex. odefun1) of the form $f\left(\Phi, \phi, \frac{d \phi}{d \Phi}\right)=0$. This algorithm solves fully implicit differential equations by inputting initial conditions (ex. $\phi_{1}$ and $\left.\left(\frac{d \phi}{d \Phi}\right)_{\pi}\right)$ and ultimately identifies an array 
of variables $\Phi, \phi$ and $\frac{d \phi}{d \Phi}$ that describe the contour of each zone within a predetermined range (ex. tspanl). This algorithm is useful for variable descriptions in zone 1 and 3, while the deformation behavior of zone 2 is evaluated using alternative approaches.

Rather than determine $\frac{d z}{d \Phi}$ in zone 2 using 'fsolve', this function directly solves for values of $\lambda_{s}$ that satisfy the system of nonlinear equations of the form $f(x)=0$ defined in fun2. A scalar value of 1 was inputted as an initial condition (x0), supposing that extension in the radial direction did not occur. Once $\lambda_{s}$ is defined, it is implemented in the evaluation of the integral,

$$
\int_{\Phi_{1}}^{\Phi_{s}} \lambda_{s} \sqrt{R_{s}^{2} \cos ^{2} \Phi+S_{s}^{2} \sin ^{2} \Phi} d \Phi,
$$

using the function 'trapz' for trapezoidal numerical integration, which ultimately determines the undeformed length of zone 2 within the interval $\left(\Phi_{\mathrm{t}}, \Phi_{\mathrm{s}}\right)$.

\subsection{Applications}

Following a detailed description of the mathematical model, it is necessary to investigate the potential applications of this model rendered in MATLAB, using deformation data collected from various platelet species. Specifically, the model predicts the membrane deformation responses for both human and murine platelets, demonstrating specific behavioral similarities between two independent micropipette aspiration studies.

To achieve this end, several MATLAB programs with compatibility for both platelet species were developed. One such program (Logarithm Model/Identification of Material Constants) satisfies the main objective of this research project, as it generates membrane material constants for the whole range of aspiration pressure $\left(0-7 \mathrm{~cm} \mathrm{H}_{2} \mathrm{O}\right)$ following the input of experimental data (human or murine). Given these material constants, another program (Logarithm Model/Simulation for Pressure Range) utilizes the initial platelet geometry and pressure range to then accurately simulate pipette aspiration. Finally, using another program (Logarithm Model/Various Results at Single Pressure), a graphical rendition of the deformed platelet shape and resultant membrane tension and stretches can also be generated. 


\subsubsection{Human Platelets}

As is potentially relevant for clinical applications, there exists a definite advantage in defining the material properties of the human platelet membrane. As such, it is appropriate to implement deformation data obtained from a previous micropipette aspiration experiment performed by Haga et al. (11), as it is extremely useful in current mathematical model applications. Although Haga et al. sought to alternatively explore the applicability of differing cell-specific models in the quantification of specific rheologic properties (i.e. Young's modulus, viscous modulus) of the platelet, the basic set of deformation data collected in this study offers adequate insight into the general deformation behavior of the platelet membrane. Specifically, Haga et al. identify the average deformation behavior of platelets $\left(\mathrm{n}=17\right.$ platelets) within a specific undeformed volume range $\left(0.33 \times 10^{-18} \mathrm{~m}^{3} \leq V_{\mathrm{o}} \leq\right.$ $1.05 \times 10^{-18} \mathrm{~m}^{3}$ ), which unfortunately fails to consider those platelets having an average and more representative volume $\left(6.0 \times 10^{-18} \mathrm{~m}^{3} \leq V_{\mathrm{o}} \leq 10 \times 10^{-18} \mathrm{~m}^{3}\right)$ (Table 2.2) (14). To address this concern, the undeformed dimensions $\left(R_{\mathrm{s}}=2.4 \mu \mathrm{m}, S_{\mathrm{s}}=0.35 \mu \mathrm{m}\right)$ and pipette radius $\left(R_{\mathrm{p}}\right.$ $=0.4 \mu \mathrm{m})$ were adjusted to accommodate an average platelet volume $\left(8.44 \times 10^{-18} \mathrm{~m}^{3}\right)$ while a set of new data points were defined to follow the average linear deformation behavior for human platelets $\left(\frac{L_{p}}{R_{p}}=10.406 \times 10^{3} p_{\mathrm{i}} \times R_{\mathrm{p}}+0.9703\right)$ obtained by Haga et al. (11). In contrast to the confirmed shape transformation outlined above (Section 4.1.2), Haga et al. (11) only presents a hypothetical diagram of platelet aspiration that depicts the unaspirated platelet fraction as discoid in shape. As such, the current work must presume that the human platelet follows an identical shape transformation to that confirmed during the aspiration of murine platelets.

In addition to the identification of membrane material constants for an average size human platelet, the mathematical model has the capacity to investigate additional behavioral characteristics. To identify the global deformation behavior of the platelet, it is pertinent to confirm whether the platelet exhibits signs of constant volume or constant area following micropipette aspiration. Using the mathematical model, then, both hypotheses can be tested by independently enforcing these model constraints and computing values for $r_{\mathrm{s}}$ that satisfy these cell responses. For example, using the constant volume hypothesis the model can 
compare values for the undeformed $\left(A_{0}\right)$ and deformed area $(A)$ and verify the validity of this assumption.

\subsubsection{Murine Platelets}

As the current mathematical model accommodates deformation data from nonspecific platelet species, one can also investigate the membrane material properties for the murine platelet, as blood samples are readily attainable. Notably, the geometric assumptions for the deformed platelet (Section 4.1.2) were validated following the real-time aspiration of murine platelets performed for this present work. Based on experimental observation, the measured mean volume for an undeformed murine platelet $(\mathrm{n}=11$ platelets $)$ is $3.088+/-1.075 \times 10^{-17}$ $\mathrm{m}^{3}$, and follows a normal distribution. To ensure the quality of subsequent analyses, model applications only implement murine platelet experimental data that maintains a linear deformation behavior and a constant volume. Using the mathematical model, then, one can isolate inadequate deformation data sets by identifying those that generate $r_{\mathrm{s}}$ values that fail to preserve volume (i.e. $V_{\mathrm{o}} \neq V$ ).

As human and murine platelets possess similar functional and structural characteristics (Section 2.1.3), one can investigate and compare the mechanical properties of the murine platelet membrane to the human alternative. Interestingly, the average deformation behavior of the murine platelet membrane $(n=11$ platelets $)$ follows the same linear trend $\left(\frac{L_{p}}{R_{p}}=0.1461 p_{\mathrm{i}} \mathrm{x} R_{\mathrm{p}}+19.923\right)$ (Section 3.2 .1$)$ as human platelets, however it exhibits greater deformation capacity. As such, the mathematical model can therefore identify a set of material constants for the murine platelet membrane that may suggest a divergence in mechanical character between species. Using the model, the controlled aspiration of a murine platelet to the point of cell lysis also offers an opportunity to potentially determine membrane strength.

\subsection{Results}

As is the main objective of the current work, one must evaluate the efficiency of the mathematical model design by confirming that the resultant material constants accurately 
predict platelet membrane mechanical responses to micropipette aspiration. To verify the validity of this model, a simulation program plotting the average theoretical deformation response of the platelet membrane was compared to membrane responses obtained experimentally. Finally, using the optimized material constants, a graphical representation of platelet aspiration was generated, along with novel mechanical responses describing membrane tension and stretch.

\subsubsection{Human Platelets}

Prior to the identification of membrane material constants, it was necessary to validate certain model constraints pertaining to the conservation of platelet volume during micropipette aspiration. As briefly outlined in Section 4.5.1, two independent conditions where either constant volume or constant area were considered and tested for their feasibility predicting natural platelet behavior to deformation. Using MATLAB and interpolated experimental data from Haga et al. (11), each condition was enforced to ultimately produce an acceptable spherical radius or ' $r_{\mathrm{s}}$ ' values using the 'fzero' function. Imposing a constant volume constraint, one is able to calculate and compare the resultant surface area $(A)$ over the specified deformation range to that of the undeformed state $\left(A_{0}\right)$. Results of this evaluation demonstrate that the platelet responds to micropipette aspiration with an average decrease in surface area by approximately $44 \%$. To illustrate this event, the decrease in areal strain during aspiration is depicted in Figure 4.5. Using the definition of the second invariant (Section 2.5.3), $I_{2}$, areal strain $\left(\frac{d A}{d A_{o}}\right)$ is equivalent to the product of the longitudinal and circumferential stretch ratios $\left(\lambda_{s} \lambda_{\theta}\right)$. 


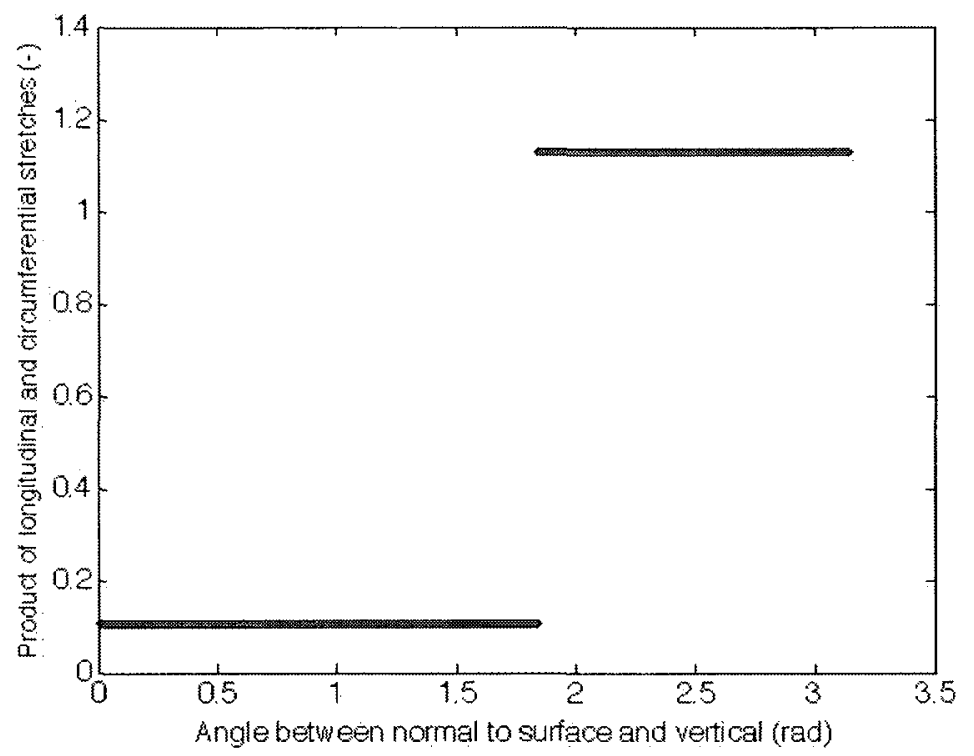

Figure 4.5: Areal strain over human platelet contour. Micropipette aspiration at $3 \mathrm{~cm} \mathrm{H}_{2} \mathrm{O}$ using a single human platelet. The platelet contour is described by $\phi$ (rad). Figure generated using the MATLAB program ' 3 Various Results at Single Pressure'.

In contrast to this condition, assuming a constant surface area yields deformed platelet volumes that indicate an average increase by approximately $148 \%$. Clearly, assuming a constant surface area is not compatible with experimental observation, as this massive volume increase is most likely indicative of cell swelling to the point of burst. Since cellular swelling was not observed, the alternative constant volume assumption was enforced in subsequent theoretical developments.

Using the mathematical model that confidently describes platelet membrane responses to micropipette aspiration, a set of material constants for the human platelet membrane was identified (Table 4.1).

Table 4.1: Material constants for an average $(n=17$ platelets $)$ human platelet membrane. Data generated using the MATLAB program ' 1 -Identification of Material Constants', $\mathrm{CI}=95 \%$.

\begin{tabular}{|c|c|c|c|c|}
\hline $\begin{array}{c}\text { Material } \\
\text { Constants } \\
\left(\mathrm{n}_{2}=3, \mathrm{n}_{3}=5\right)\end{array}$ & $\mathrm{c}_{1}(\mathrm{~N} / \mathrm{m})$ & $c_{2}(-)$ & $c_{3}(-)$ & $\mathrm{T}_{\mathrm{o}}(\mathrm{N} / \mathrm{m})$ \\
\hline $\begin{array}{c}\text { Human Platelet } \\
\text { Membrane }\end{array}$ & $\begin{array}{c}4.69999294 \\
\pm \\
0.0071118\end{array}$ & $\begin{array}{c}0.00014698 \\
\pm \\
0.00000461\end{array}$ & $\begin{array}{c}-0.00001802 \\
\pm \\
0.00000384\end{array}$ & $\begin{array}{c}-0.00008445 \\
\pm \\
0.00000414\end{array}$ \\
\hline
\end{tabular}

The material constants $\left(c_{i}, i=1-3\right)$ promote positive tension in the membrane during deformation, as evidenced in constitutive equations (Section 4.3), while membrane prestress suggests that the load-free membrane is under compression. 
To verify the accuracy of these material constants in generating adequate theoretical predictions of platelet membrane deformation, the average theoretical deformation behavior $\left(\frac{L_{p}}{R_{p}}\right.$ vs. $\left.p_{\mathrm{i}} \mathrm{x} R_{\mathrm{p}}\right)$ was plotted in contrast to the experimental alternative (Figure 4.6).

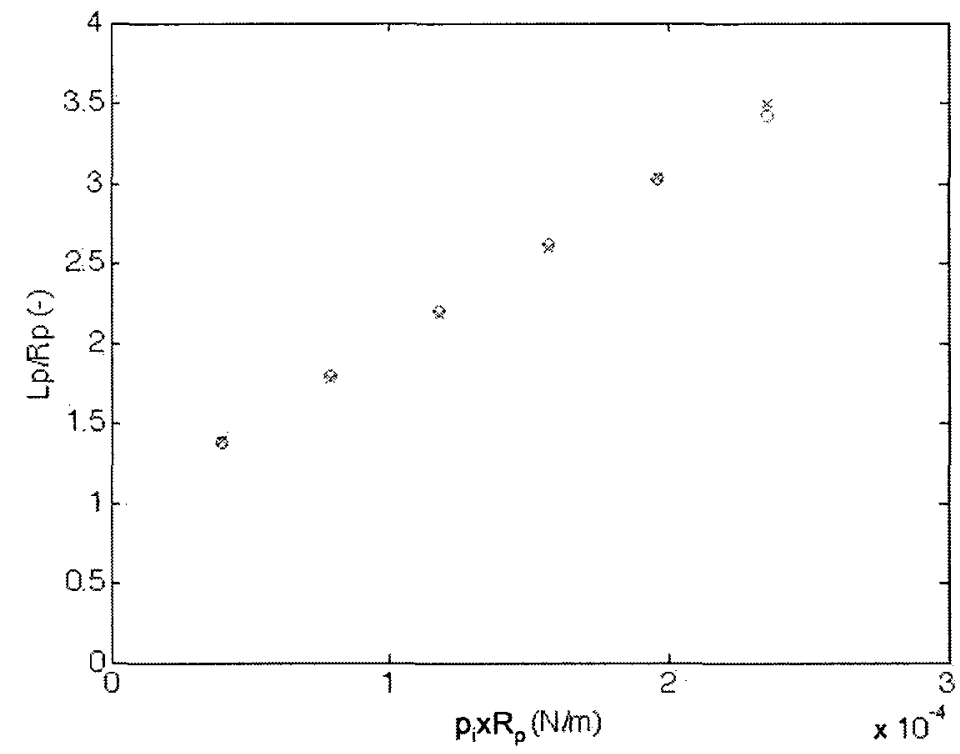

Figure 4.6: Average theoretical (x) vs. experimental (o) deformation behavior for the human platelet membrane. This relationship is represented by plotting the product of aspiration pressure $\left(p_{i}\right)$ and pipette tip radius $\left(R_{\mathrm{p}}\right)$ versus the quotient of aspired platelet length $\left(L_{\mathrm{p}}\right)$ and pipette tip radius. Figure generated using the MATLAB program '2-Simulation for Pressure Range'.

The accuracy of these constants were then exploited, along with definitions of geometry and a pressure range, to illustrate micropipette aspiration of a human platelet (Figure 4.7): 
(a)

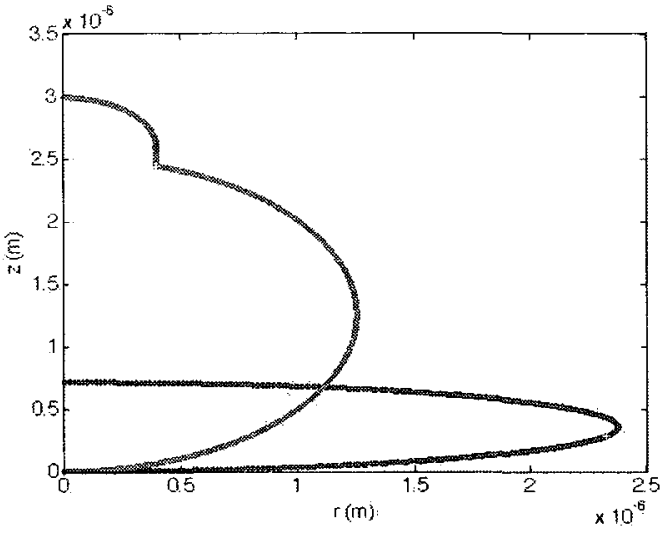

(c)

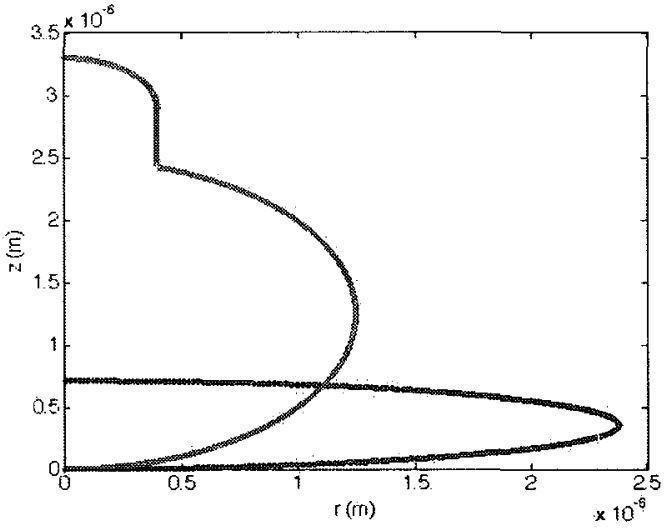

(b)

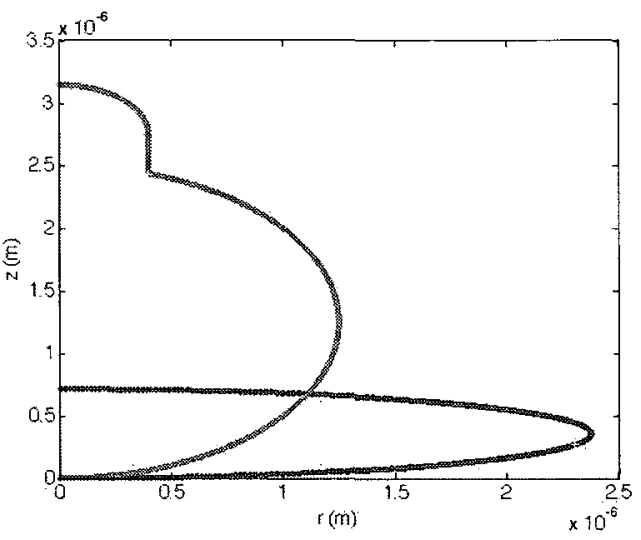

(d)

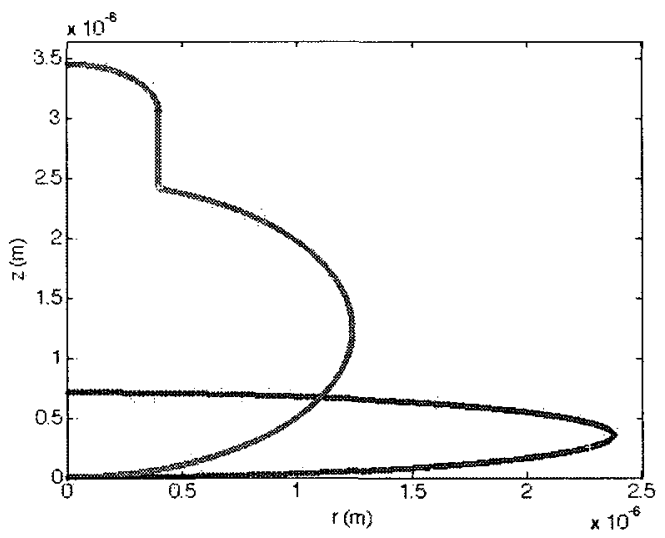

Figure 4.7: MATLAB simulation of an aspired human platelet. A single resting platelet was aspired over a pressure gradient of $1-4 \mathrm{~cm} \mathrm{H}_{2} \mathrm{O}\left(\sim 98-392 \mathrm{~N} / \mathrm{m}^{2}\right)$ (Figures 4.7, a-d). Undeformed (discoid) and deformed platelet shapes are shown. Figure generated using the MATLAB program ' 3 -Various Results at Single Pressure'.

As depicted above, the inactive human platelet has limited deformation capacity, with a maximum aspirated volume $\left(p_{\mathrm{i}}=7 \mathrm{~cm} \mathrm{H}_{2} \mathrm{O}\right)$ reaching approximately $7.4 \%$ of the total platelet volume. The deformation state of the human platelet membrane can also be described in terms of the resultant membrane tension, whose magnitude is maintained over the entire platelet contour and increases linearly with respect to aspiration pressure (Figure 4.8). 


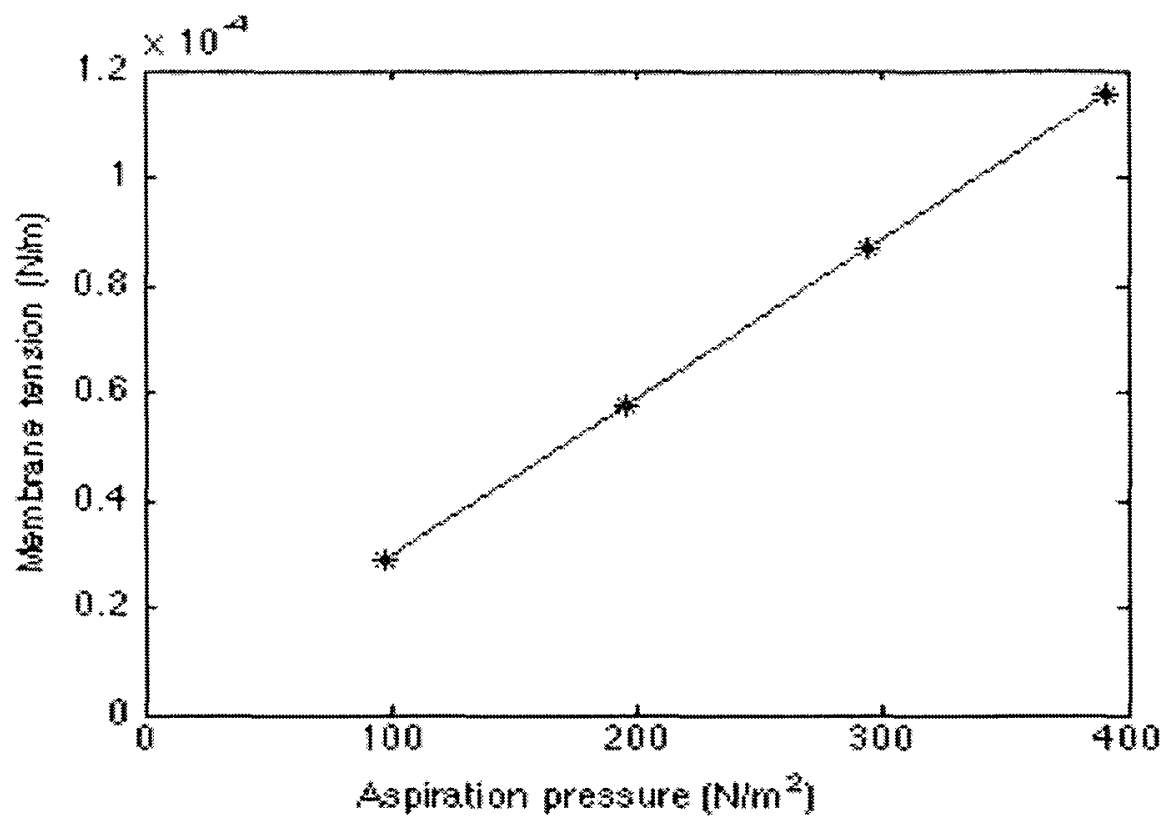

Figure 4.8: Tension accumulation in the human platelet membrane. Figure generated using the MATLAB program '3-Various Results at Single Pressure'.

The material constants also prove useful in the characterization of membrane stretch ratios in the longitudinal $\left(\lambda_{s}\right)$ and circumferential $\left(\lambda_{\theta}\right)$ directions, while also emphasizing the constraining effects of the micropipette (Figure 4.9).

(a)

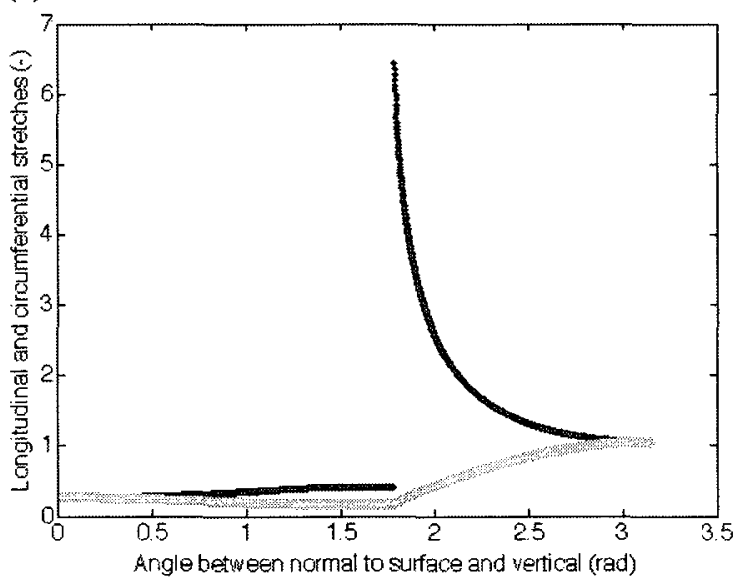

(b)



Figure 4.9: Longitudinal ( $\lambda_{s}$, black) and circumferential $\left(\lambda_{\theta}\right.$, grey) stretch ratios over human platelet contour for an aspiration pressure of (a) $1 \mathrm{~cm} \mathrm{H} \mathrm{H}_{2} \mathrm{O}$ and (b) $4 \mathrm{~cm} \mathrm{H}_{2} \mathrm{O}$. The platelet contour is described by $\phi$ (rad). Figure generated using the MATLAB program '3-Various Results at Single Pressure'.

Considering the circumferential $\left(\lambda_{\theta}\right)$ and longitudinal $\left(\lambda_{s}\right)$ stretch ratios, this membrane behavior is segmented into zone $1\left[(\pi-\varepsilon), \Phi_{\mathrm{s}}\right]$, zone $2\left[\Phi_{\mathrm{s}}, \Phi_{\mathrm{t}}\right]$ and zone $3\left[\Phi_{\mathrm{t}}, \varepsilon\right]$. 
Interestingly, with an appreciable decrease in circumferential stretch across these zones ( 1 to 3), the membrane appears to adapt to the rigid geometry of the micropipette. Specifically, the confines of the micropipette are most significant in zone 2 , as circumferential stretch is limited in this region. In contrast, circumferential stretch in the membrane increases with proximity to $\pi-\varepsilon$, as it describes the transition from the discoid to spherical shape. The circumferential stretch behavior of the hemispherical cap (zone 3) also emphasizes its diminutive size when compared to the external sphere (zone 1). Regardless of aspiration pressure, the volume of the hemispherical cap remains constant and dependent on the pipette radius, $R_{\mathrm{p}}$. Interestingly, maintaining hemispherical cap volume fails to complement the stretch behavior in this zone, as both $\lambda_{\theta}$ and $\lambda_{s}$ appear to increase slightly with applied pressure. Finally, the aspiration-induced shape transformation in zone 1 generates a marked increase in longitudinal stretch towards the pipette tip $(\phi \approx 1.78 \mathrm{rad})$, which steadily decreases with the approach of the opposite pole $(\phi=0 \mathrm{rad})$. At micropipette entry, a significant discontinuity in $\lambda_{s}$ for the human platelet membrane is also relevant in future discussions.

Although inconclusively, Haga et al. (11) observed a separation of the aspirated portion of the platelet from the external platelet fraction with aspiration pressures exceeding $6 \mathrm{~cm} \mathrm{H}_{2} \mathrm{O}$. This lytic event can therefore be used as a means to investigate membrane strength, by relating membrane tension to some incompatible magnitude of aspiration pressure. Inputting an applied negative pressure of $7 \mathrm{~cm} \mathrm{H}_{2} \mathrm{O}$ in the MATLAB program 'Logarithm Model/Various Results at Single Pressure' generated a hypothetical strength of $2.0364 \times 10^{-4} \mathrm{~N} / \mathrm{m}$ for the human platelet membrane. Manipulating the above program, membrane strength also proves consistent with variations in material constants, as independent evaluations of constants at the limits of confidence intervals (i.e. $c_{i} \pm \mathrm{CI}$ ) generated negligible effects on the magnitude of tensile strength.

\subsubsection{Murine Platelets}

As an extension of the mathematical model, the current work also investigated the mechanical properties of the murine platelet membrane. To begin, murine platelet deformation responses were implemented to confirm whether platelet volume was also maintained. Using the same rationale, conserving murine platelet volume during 
deformation proved compatible with experimental observation, and demonstrated an average decrease in membrane surface area by approximately $25 \%$. To illustrate this event, the increase in areal strain during aspiration is depicted in Figure 4.10.

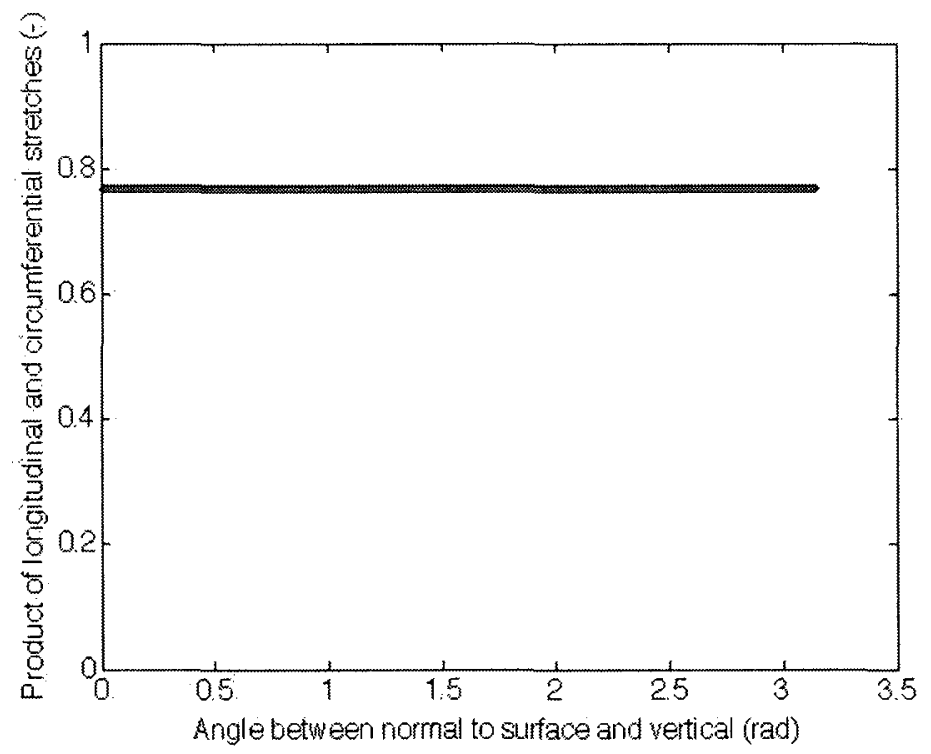

Figure 4.10: Areal strain over murine platelet contour. Micropipette aspiration at $3 \mathrm{~cm} \mathrm{H}_{2} \mathrm{O}$ using a single murine platelet. The platelet contour is described by $\phi(\mathrm{rad})$. Figure generated using the MATLAB program ' 3 Various Results at Single Pressure'.

Evidently, murine platelets respond to micropipette aspiration by maintaining area strain over its contour. In contrast to human platelet deformation responses, the murine membrane appears to accommodate large aspiration lengths $\left(L_{\mathrm{p}}\right)$, which may indicate different mechanical properties. As such, experimental data describing the average deformation response of a murine platelet was applied to the model, and successfully generated a set of membrane material constants (Table 4.2). To identify these membrane material constants for the mouse, one must first define a scalar relationship between the major and minor radii of the undeformed platelet, as described by the label 'shaperatio' in MATLAB (Logarithm Model/Identification of Material Constants/main). This ratio describes the radial dimensions of the undeformed human platelet, and ranges between 0.15 and 0.25 (14). For the murine model, the effect of increasing this shaperatio was investigated, as it could potentially have a significant effect on the circumferential and longitudinal stretch values (Logarithm Model/Identification of Material Constants/errLC), and ultimately the vectors of $\Phi$ and $\phi$ describing zones 1 and 3 . Fortunately, redefining the size of the undeformed platelet (shaperatio $=0.15$ or 0.25 ) had negligible effects on the set of material constants produced. 
Table 4.2: Material constants for an average $(n=11$ platelets $)$ murine platelet membrane. Data generated using the MATLAB program ' 1 -Identification of Material Constants', $(\mathrm{CI}=95 \%)$.

\begin{tabular}{|c|c|c|c|c|}
\hline $\begin{array}{c}\text { Material Constants } \\
\left(\mathrm{n}_{2}=1, \mathrm{n}_{3}=3\right)\end{array}$ & $\mathrm{c}_{1}(\mathrm{~N} / \mathrm{m})$ & $\mathrm{c}_{2}(-)$ & $\mathrm{c}_{3}(-)$ & $\mathrm{T}_{\mathrm{o}}(\mathrm{N} / \mathrm{m})$ \\
\hline & $\begin{array}{c}3.89000058 \\
\pm\end{array}$ & $\begin{array}{c}0.00018242 \\
\pm\end{array}$ & $\begin{array}{c}0.00001021 \\
\pm\end{array}$ & $\begin{array}{c}-0.00049714 \\
\pm\end{array}$ \\
$\begin{array}{c}\text { Murine Platelet } \\
\text { Membrane }\end{array}$ & $\begin{array}{c} \pm .00000592 \\
0.00000036\end{array}$ & $\begin{array}{c}0.00000016 \\
0\end{array}$ & \begin{tabular}{c}
0.00000126 \\
\hline
\end{tabular} \\
\hline
\end{tabular}

Similarly, the murine material constants $\left(c_{i}, i=1-3\right)$ promote positive tension in the membrane during deformation, as evidenced in constitutive equations (Section 4.3), while membrane prestress suggests that the load-free membrane is under compression. Further, murine membrane prestress appears to maintain compression with an approximate six-fold increase in magnitude compared to the human membrane.

The predictive capabilities of these material constants were also verified by comparing the average theoretical deformation behavior $\left(\frac{L_{p}}{R_{p}}\right.$ vs. $\left.p_{\mathrm{i}} \mathrm{x} R_{\mathrm{p}}\right)$ to the experimental alternative (Figure 4.11).

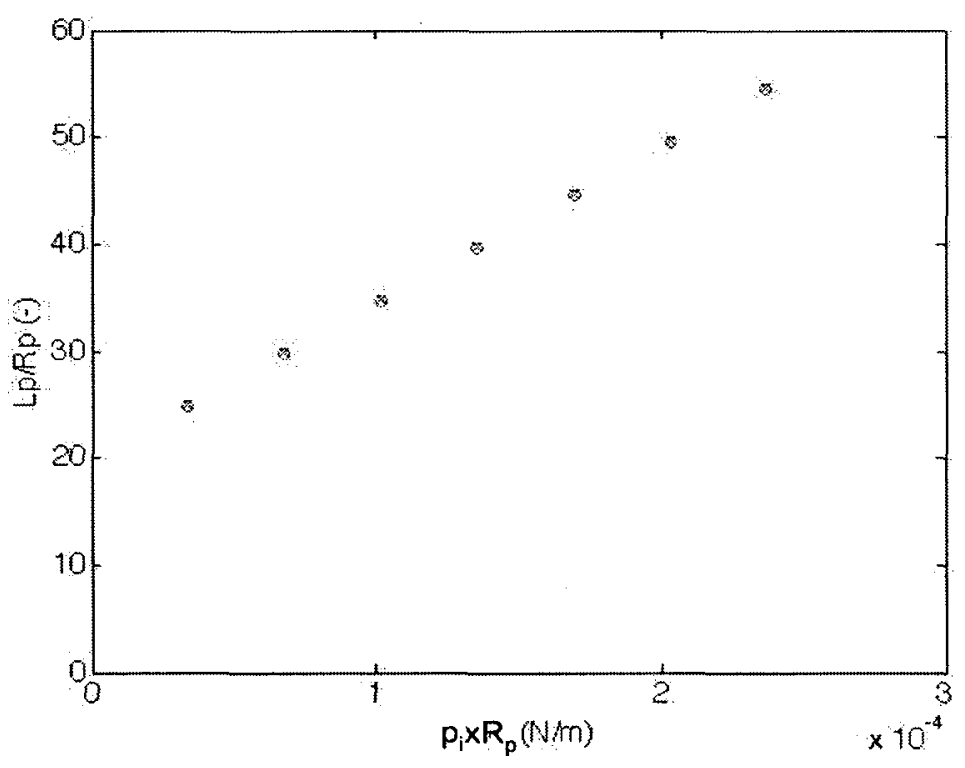

Figure 4.11: Average theoretical (x) vs. experimental (o) deformation behavior for the murine platelet membrane. This relationship is represented by plotting the product of aspiration pressure $\left(p_{i}\right)$ and pipette tip radius $\left(R_{\mathrm{p}}\right)$ versus the quotient of aspired platelet length $\left(L_{\mathrm{p}}\right)$ and pipette tip radius. Figure generated using the MATLAB program '2-Simulation for Pressure Range'.

Once again, the integrity of the model, defined by its ability to generate accurate material constants for nonspecific platelet species, appears sound. As the membrane material 
constants for the murine platelet generate an accurate theoretical deformation description, they proved useful in generating a pressure-dependent illustration of murine platelet aspiration (Figure 4.12):

(a)

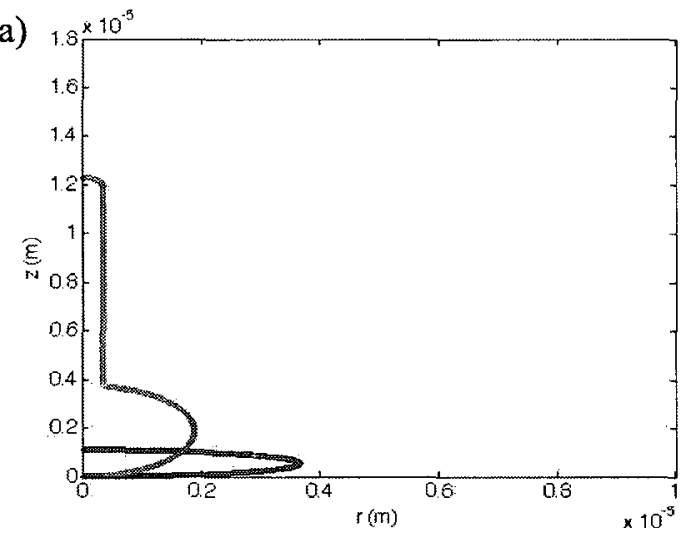

(c)

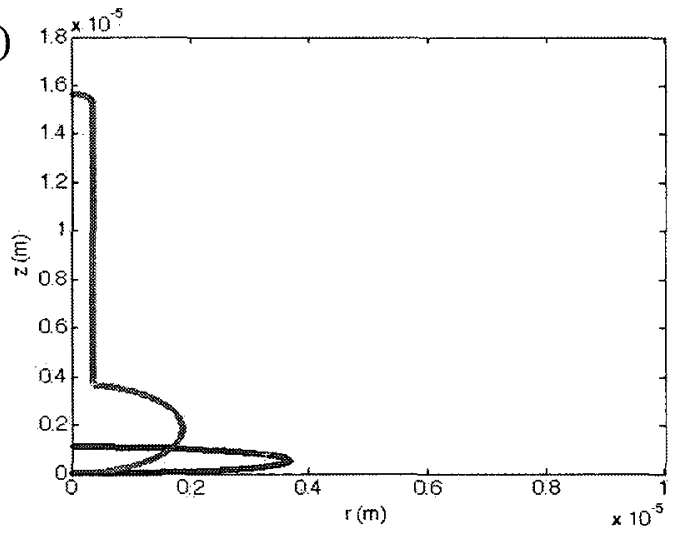

(b)

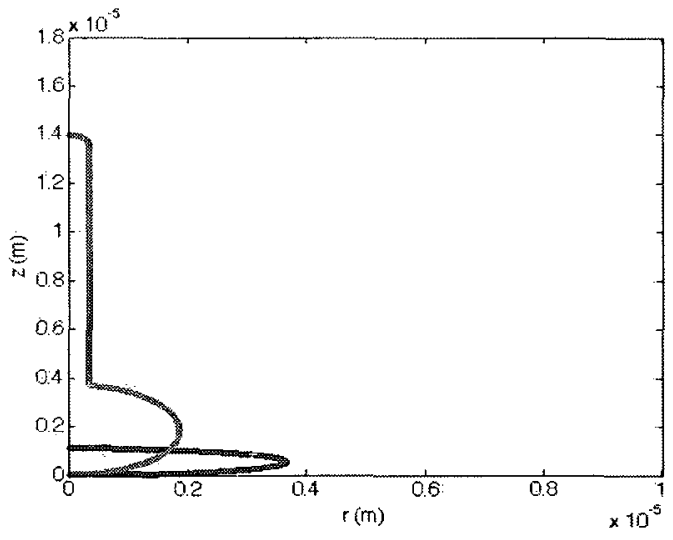

(d)

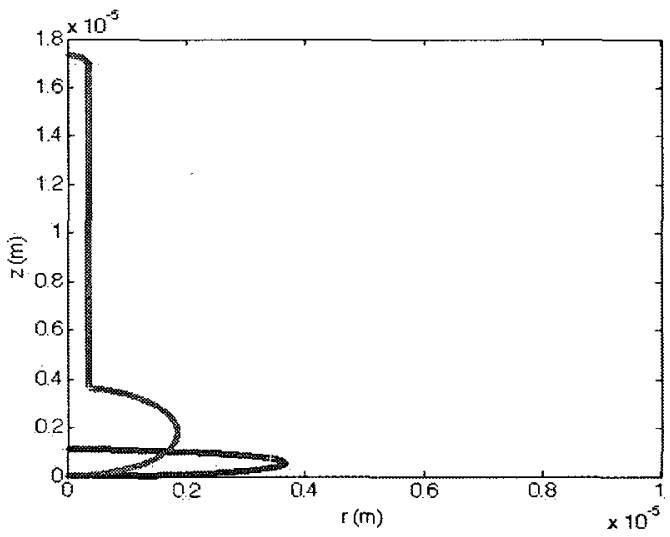

Figure 4.12: MATLAB simulation of an aspired murine platelet. A single resting platelet was aspired over a pressure gradient of $1-4 \mathrm{~cm} \mathrm{H}_{2} \mathrm{O}\left(\sim 98-392 \mathrm{~N} / \mathrm{m}^{2}\right)$ (Figures 4.12, a-d). Undeformed (discoid) and deformed platelet shapes are shown. Figure generated using the MATLAB program '3-Various Results at Single Pressure'.

As depicted above, the inactive mouse platelet has increased deformation capacity when compared to the human platelet, with its maximum aspirated volume reaching approximately $23 \%$ of the total platelet volume. The deformability of the murine platelet membrane is also apparent in descriptions of resultant tension, as the membrane accommodates aspiration at comparably lower tension magnitudes to that of the human platelet. Membrane tension also increases linearly with respect to aspiration pressure (Figure 4.13), and is maintained over the entire platelet contour. 


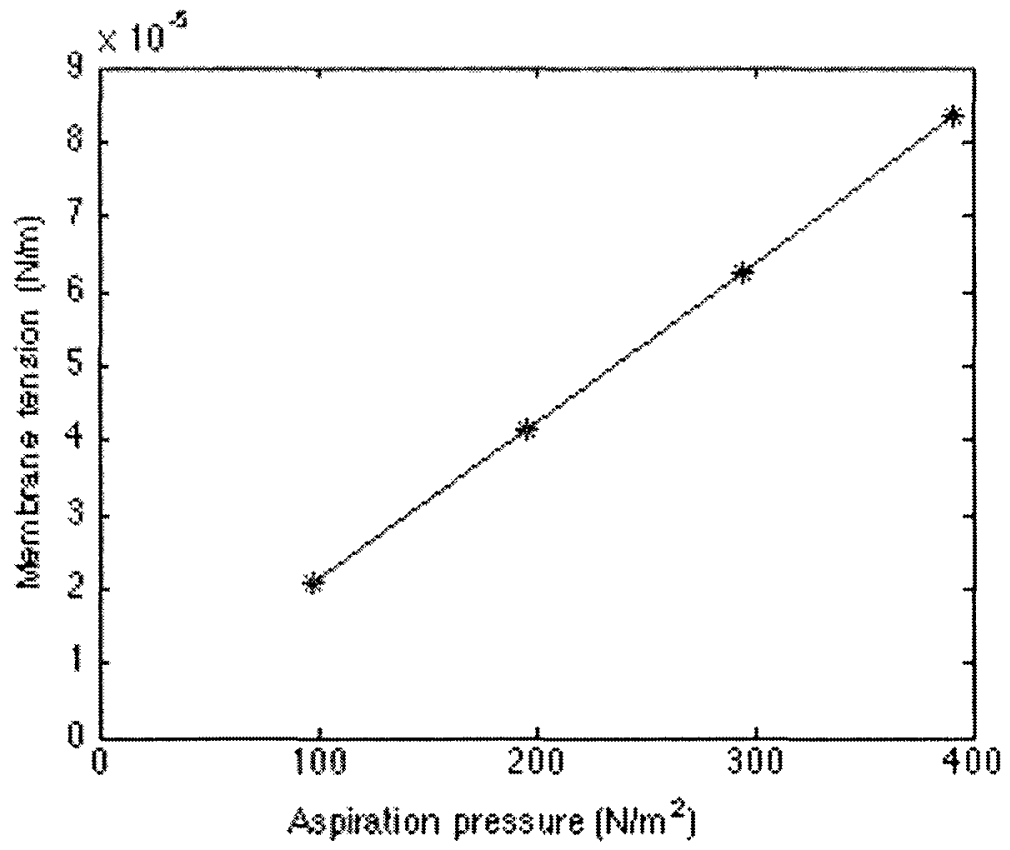

Figure 4.13: Tension accumulation in the murine platelet membrane. Figure generated using the MATLAB program '3-Various Results at Single Pressure'.

The material constants also prove useful in the characterization of membrane stretch ratios in the longitudinal $\left(\lambda_{s}\right)$ and circumferential $\left(\lambda_{\theta}\right)$ directions, while also emphasizing the constraining effects of the micropipette (Figure 4.14).

(a)

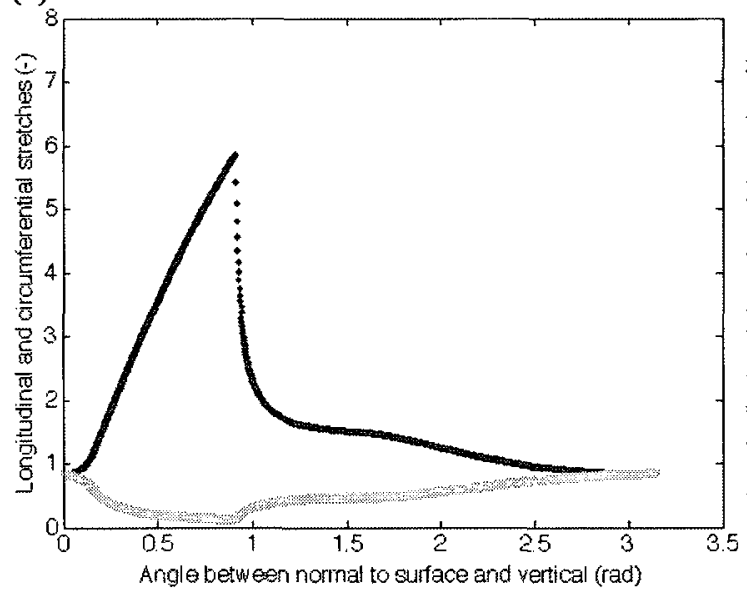

(b)

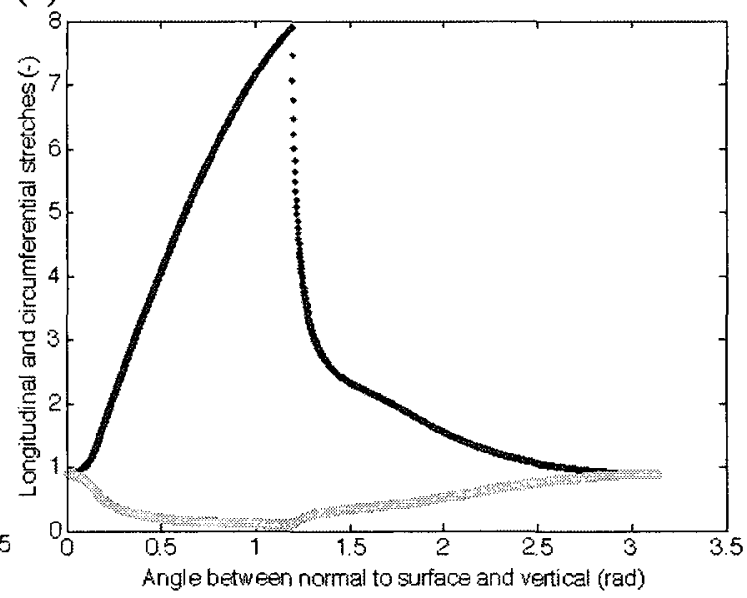

Figure 4.14: Longitudinal $\left(\lambda_{s}\right.$, black) and circumferential $\left(\lambda_{\theta}\right.$, grey) stretch ratios over murine platelet contour for an aspiration pressure of (a) $1 \mathrm{~cm} \mathrm{H} \mathrm{H}_{2} \mathrm{O}$ and (b) $4 \mathrm{~cm} \mathrm{H} \mathrm{H}_{2} \mathrm{O}$. The platelet contour is described by $\phi$ (rad). Figure generated using the MATLAB program '3-Various Results at Single Pressure'.

Clearly, the above description of membrane stretch complements the observed deformation responses of the murine platelet, as a large volume fraction of the murine platelet succumbs 
to large aspiration pressures. One must also appreciate that the range of stretch descriptions $\left(\lambda_{s}, \lambda_{\theta}\right)$ in zone 1 will decrease slightly with increasing aspiration pressure, as the platelet majority will occupy the inside of the micropipette (Figure 4.14 , b). In general, the circumferential membrane stretch tapers in magnitude as it diverges from a spherical shape and approaches the cylindrical shape of micropipette tip $(\phi \approx 0.90 \mathrm{rad})$, whereupon the confines of the glass micropipette limit the circumferential stretch capacity. Finally, a noticeable increase in $\lambda_{\theta}$ is achieved in zone 3 (hemispherical cap), where the membrane follows a similar circumferential stretch profile (sphere) to that of the bottom pole. Regardless of aspiration pressure, however, the volume and stretch behavior in this region remains constant and dependent on the pipette radius, $R_{\mathrm{p}}$. Alternatively, the longitudinal stretch response in zone 1 indicates a lengthening of the membrane, as the unaspirated platelet fraction follows the transition from a discoid $\left(R_{\mathrm{s}} \neq S_{\mathrm{s}}\right)$ to spheroid. Overall, the stretch capabilities of the murine platelet membrane prove larger than those of the human platelet.

Finally, the current work sought to investigate membrane strength by first identifying those murine platelet aspiration events where the membrane ruptured. Unfortunately, evidence of membrane rupture is limited, however preliminary results obtained using the MATLAB program 'Logarithm Model/Various Results at Single Pressure' have related an applied aspiration pressure $\left(8 \mathrm{~cm} \mathrm{H}_{2} \mathrm{O}\right)$ to a tension limitation in the membrane $\left(1.6808 \times 10^{-4}\right.$ $\mathrm{N} / \mathrm{m}$ ) where membrane integrity is compromised. As with the human platelets, membrane strength for the murine platelet also proves consistent with variations in material constants, as independent evaluations of constants at the limits of confidence intervals (i.e. $\mathrm{c}_{\mathrm{i}} \pm \mathrm{CI}$ ) generated negligible effects on the magnitude of tensile strength. To confidently identify membrane strength, however, a more robust sample size is necessary, as murine platelet lysis only occurred for 2 platelets of the sample population that maintained constant volume and a linear deformation behavior ( $\mathrm{n}=11$ platelets). 


\section{Chapter 5}

\section{Discussion}

The subsequent analysis first addresses the general characteristics of the model and its divergence from previous membrane models. Then, as a complement to this development, model solutions are discussed for their capacity to predict and simulate membrane behavior during micropipette aspiration. The justification of compressive residual stress is also necessary, with an opportunity to outline model deficits and potential improvements.

\subsection{General Model}

During the preliminary stages of model development, it was necessary to explore the applicability of alternative, well-established cell models, with specific interest to those characterizing the deformation responses of erythrocyte membranes $(94,98)$. As detailed in Section 2.5.3, one such model defined a set of constitutive equations describing erythrocyte membrane responses to induced sphering while accounting for negligible areal strain (98). The complementary efforts of Skalak et al. (94) sought to extend this deformation description using a novel strain energy function $(W)$, which imposed specific geometric constraints to the erythrocyte shape transformation that limited applicability to other cellular structures. Specifically, the deformed erythrocyte can only maintain a spherical shape $\left(T_{\mathrm{s}}=T_{\theta}\right)$ when the material constant $c_{1}$ has a magnitude of zero. Preliminary model applications implementing Skalak's strain energy function $(W)$ and Evans' derived stress-strain relationship using human platelet deformation data proved inaccurate, as the mathematical model produced material constants that failed to accurately simulate the spherical shape transformation by producing nonzero values for the material constant $c_{1}$. Further, the deformation responses of the human platelet membrane prove incompatible with the erythrocyte membrane model, as it deforms with significant areal strain. As such, an alternative phenomenological model (Section 4.2 - 4.3) fit to accommodate platelet deformation data was introduced, and ultimately predicted membrane deformation behavior with good accuracy. 
In terms of structural complexity, the model employs a continuum mechanics approach to platelet membrane descriptions. Specifically, then, only the global mechanical character of the platelet is considered, treating the deformed cell as a hyperelastic shell enveloping a liquid cytoplasm. Since the structure of platelets possesses the stabilizing advantage of a complex cytoskeleton (14), one must appreciate that the current model offers a simplistic explanation of the continuum mechanical behavior of platelet membranes, irrespective of these underlying contributions (118). The mathematical model also limited its material model descriptions to one describing the behavior of an isotropic material. This simplistic material concept is one supported by static deformation data collected from murine platelet aspiration experiments (Section 3.1.4), as membrane deformation behavior was uniform and independent of aspiration location. The model also indirectly defines an acceptable range of undeformed platelet volumes for both platelet species, as dictated by the radial dimensions of the platelet (Section 4.4.3). Testing the extremes (shaperatio $=0.15-$ 0.25) of volume variability, there was no significant change in membrane mechanical behavior (i.e. material constants, deformability) (66) for either platelet species.

Further, the theoretical foundation of this model requires a collaborative effort that validates assumptions using well-established observations and membrane deformation behaviours for both human and murine platelet aspiration experiments. Using aspiration data for each platelet species, along with a well-defined geometric concept of an aspirated platelet, one MATLAB program generates a set of optimized material constants that can be used to describe the behaviour of the platelet over the entire aspiration pressure range. In conjunction with the platelet's initial geometry and pressure range, these constants can be used to predict the state of platelet membrane deformation and stress. Further, the development of a program that successfully simulates micropipette aspiration offers novel insight into the mechanical behaviour of the platelet membrane, where one can investigate both the tension and stretch over the membrane contour for a wide range of negative pressures $\left(0-7 \mathrm{~cm} \mathrm{H}_{2} \mathrm{O}\right)$. Aspiration simulations based on experimental data inputs also validate the conservation of volume assumption. 


\subsection{Human Platelets}

As mentioned in section 4.5.1, the model only implements static deformation data that follows a linear behavior (as described by $\frac{L_{p}}{R_{p}}$ vs. $p_{\mathrm{i}} \mathrm{x} R_{\mathrm{p}}$ ), which is independent of platelet size. Specifically, the study by Haga et al. (11) identified this deformation trend for platelets at the lower end of the volume spectrum $\left(0.33 \times 10^{-18} \mathrm{~m}^{3} \leq V_{\mathrm{o}} \leq 1.05 \times 10^{-18} \mathrm{~m}^{3}\right)$, which fails to address the deformation behavior of human platelets with an average volume $\left(6.0 \times 10^{-18}\right.$ $\mathrm{m}^{3} \leq V_{\mathrm{o}} \leq 10 \times 10^{-18} \mathrm{~m}^{3}$ ), as dictated by literature (14). As such, the initial platelet volume was adjusted to comply with this average volume range, and a new set of deformation data points were interpolated using the linear trend defined by Haga et al. (11). Obviously, the applicability of this deformation behavior to large platelet volumes is uncertain, as this is not a definitive trend. One must therefore appreciate that this assumption is merely speculative, but gains some measure of validity when one considers the similar linear deformation trend observed for murine platelet membrane for a large range of volumes $\left(1.25 \times 10^{-17} \mathrm{~m}^{3} \leq V_{\mathrm{o}} \leq\right.$ $\left.4.13 \times 10^{-17} \mathrm{~m}^{3}\right)$. Further, additional aspiration studies $(86,87)$ support this deformation behavior, but only present the average initial extension length $\left(L_{\mathrm{p}}\right)\left(p_{\mathrm{i}}=1 \mathrm{~cm} \mathrm{H}_{2} \mathrm{O}, R_{\mathrm{p}}=0.35\right.$ $\mu \mathrm{m})$ rather than the average volume of their human platelet model. Comparing these length values, then, one can speculate that the average platelet volumes in these studies fall in a larger range to those used by Haga et al., as $L_{\mathrm{p}}$ values are an order of magnitude larger (Burris et al. (86): $L_{\mathrm{p}}=2.16 \pm 0.12 \mu \mathrm{m}$; Burris et al. (87): $L_{\mathrm{p}}=1.15 \pm 0.13 \mu \mathrm{m}$; Haga et al. (11): $0.47 \mu \mathrm{m}$ ).

Using this synthesized deformation data for human platelets the model was able to resolve specific uncertainties regarding general membrane deformation behavior. Specifically, the theoretical conservation of an average platelet volume during deformation proves compatible with experimental observation. Alternatively, deforming a human platelet at constant surface area results in a significant increase in cell volume $\left(\sim 148 \%\right.$ of $\left.V_{\mathrm{o}}\right)$ that challenges physiological norms. Essentially, this deformation condition may induce extreme membrane instability that could potentially induce cell bursting. Qualitative descriptions of human platelet aspiration by Haga et al. (11) presented no such events. An acceptable solution is therefore one that conserves volume with a significant decrease in areal strain. Theoretically, micropipette aspiration may induce a reversible compression of glycocalyx 
glycoproteins and the phospholipid bilayer, causing a decrease in areal strain. This condensing effect lacks validation, however, as the current work focuses on global membrane responses alone. Analyzing the zone distribution of areal strain $\left(\frac{d A}{d A_{o}}\right)$ depicted in Figure 4.5, however, one might identify a general location where a decrease in areal strain is most prominent. Notably, the magnitude of areal strain appears to be limited by the confines of micropipette (zones 2,3), but slowly approaches that achieved in zone 1 with increasing aspiration pressure. Preceding this evaluation of areal strain, the rigid micropipette limits the extension capabilities of the membrane in the longitudinal $\left(\lambda_{s}\right)$ and circumferential $\left(\lambda_{\theta}\right)$ directions. As detailed in Section 4.6.1, the human platelet membrane actively extends most prominently in regions lacking rigid constraints. Upon entry into the micropipette, circumferential stretch is significantly limited by the micropipette, reaching a limit at $r=R_{\mathrm{p}}$. With an increase in applied pressure, the magnitude of $\lambda_{\theta}$ in zone 3 also increases as $\phi$ approaches 0 , supporting the slight progression of the platelet into the micropipette. Alternatively, longitudinal membrane extension, whose magnitude increases along the axis of symmetry ( $z$-axis), is dependent on applied negative pressure $\left(p_{\mathrm{i}}\right)$. The significant $\lambda_{\mathrm{s}}$ decrease in zone 2 is therefore most prominent at low aspiration pressures, however this stretch response would become more prominent as the aspirated volume fraction increases along with applied negative pressure (Figure 4.9, a, b). To justify the significant discontinuity of $\lambda_{\mathrm{s}}$ values at the junction defining zone 1 and 2 , one must consider the distinctive manner in which $\lambda_{s}$ is evaluated for zone 2. As detailed in Section 4.4.1, one cannot directly solve for $\lambda_{s}$, as $\frac{d z}{d \Phi}$ is unknown. Alternatively, acceptable $\lambda_{s}$ values for zone 2 , along with membrane material constants, are iteratively adjusted to conserve platelet volume during deformation. Although meeting this volume constraint, the resultant $\lambda_{s}$ values for the human platelet membrane may therefore be lacking in their capacity to maintain continuity between zones 1 and 2 .

Following the general descriptions of membrane deformation behavior, the model identifies a set of material constants (Table 4.1) that satisfy the main objectives of this work. First, the material constants generate a theoretical behavioral prediction that follows almost identically the linear deformation response observed experimentally (Figure 4.6). The 
theoretical illustration of human platelet micropipette aspiration also demonstrates the consistency of these material constants in predicting membrane deformation responses. Clearly, this simulation offers visual proof of the limited deformation capacity of the human platelet membrane, which could theoretically depend on platelet ultrastructure. As cited by White et al. (66), the "lengths of aspirated segments could reflect the protein-lipid character of the surface membrane attachment of the membrane to the underlying cytoskeletal network, the state of organization of mobile microfilamentous structures of the cytoskeleton, or integrity of the circumferential band of microtubules." The model also details the significant but debatable shape contrast between the undeformed and deformed shape, as segmented by zones (Figure 4.7). That is, previous aspiration studies using human platelets present conflicting evidence of the unaspirated external geometry, loosely suggesting either a discoid shape $(86,11)$ or one that transforms to a spheroid shape (66). Ultimately, the current model presumes functional similarities between human and murine platelet species (Section 2.1.3), thus committing to an external spherical shape that mimics the observed aspiration responses of murine platelets. Further, a study by White et al. $(65,66)$ independently supports findings from the current murine platelet aspiration work, demonstrating the irrelevance of aspiration location on membrane responses. To justify this behavior, White et al. $(65,66)$ postulates a "uniform distribution of forces" as the governing factor of membrane deformability. Implementing the optimized material constants for the human platelet membrane, one MATLAB program confirms these uniformity suppositions, as membrane tension positively correlates with applied pressure and appears constant over the entire statically deformed membrane. This consistent tension may also lend to a potential uniform distribution of reinforcing and underlying actin filaments, as "factors other than the coiled microtubule band appear to influence cell surface tension or translate the force of the tubules to the surface membranes" (66).

Lastly, the mathematical model offers speculative insight into membrane strength, as Haga et al. (11) presents a preliminary range of aspiration pressures $\left(<6 \mathrm{~cm} \mathrm{H}_{2} \mathrm{O}\right)$ that may trigger human platelet membrane rupture. It is important to recognize the general uncertainty of these observations, as Haga et al.'s work fails to directly explore the limitations of the membrane, as only platelets which passively released from pipette tip were deemed useful (11). Further, similar aspiration experiments present conflicting evidence of membrane 
deformability, citing the safe aspiration and subsequent release of platelets at aspiration pressures of $10 \mathrm{~cm} \mathrm{H}_{2} \mathrm{O}(66)$.

\subsection{Murine Platelets}

Although murine platelet membranes demonstrate greater deformation capacity and appear to function at larger volumes, the fundamental similarities between platelet species prove sufficient for the identification of murine material constants using the same approach. Notably, the experimentally observed average murine platelet volume exceeds the average volume range outlined in literature (Section 2.1.3). This divergence, however, is most likely a result of variability in the mouse strain and size investigated. In terms of average deformation behavior, the murine platelet membrane follows a linear increase in extension length that parallels human platelet membrane responses to aspiration pressure. The linear deformation behaviour of the murine platelet membrane provides experimental validation for a simplistic material behaviour (i.e, isotropic) assumption, as this behavioural trend proves independent of aspiration location (Section 3.2.2). This murine data set was subsequently implemented, along with different inputs for $\mathrm{n}_{2}$ and $\mathrm{n}_{3}$, using the same mathematical model to obtain membrane material constants (Table 4.2). Relative to the human material constants, the smaller magnitudes of the murine alternative appears to support a behavioral discrepancy in membrane deformability, potentially indicating a decrease in murine membrane stiffness. Notably, both sets of material constants also present evidence of compressive residual stress $\left(T_{\mathrm{o}}\right)$ in the membrane, but is most prominent in murine platelets. The significance of this unloaded stress state will be detailed in Section 5.4. In terms of predictive capabilities, these constants prove equally effective in generating theoretical simulations of average deformation behavior (Figure 4.11) and graphical depictions of platelet aspiration (Figure 4.12). Similar to human membrane responses, the conservation of murine platelet volume also results in a surface area decrease. Markedly, this areal deviation from the undeformed state proves less significant $(-18 \%)$ than the human response $(-44 \%)$, while the model also presents evidence of uniform areal strain over the murine platelet contour (Figure 4.10). To rationalize the latter event where areal strain uniformly distributed, one might also consider the extension capabilities of the membrane (Figure 4.14). In contrast to the human alternative, a larger volume fraction of the murine platelet is aspirated, thereby improving the 
uniformity of extension descriptions $\left(\lambda_{s}, \lambda_{\theta}\right)$ and resultant areal strain $\left(\frac{d A}{d A_{o}}=\lambda_{s} \lambda_{\theta}\right)$ across the contour.

In addition, the model is able to resolve a similar description of murine membrane tension at static equilibrium to that generated in the human membrane. Although murine membrane tension manifests at lower magnitudes, it follows the same uniform distribution over the membrane contour. Evidently, the murine platelet's increased deformability infers a minor tension response following an applied pressure. Finally, an assessment of membrane strength demonstrates a relative consistency between human $\left(2.0365 \times 10^{-4} \mathrm{~N} / \mathrm{m}\right)$ and murine $\left(1.6808 \times 10^{-4} \mathrm{~N} / \mathrm{m}\right)$ membrane tension limitations. Although preliminary in nature, this relative consistency in membrane strength supports functional homology between platelet species, while also contributing novel mechanical descriptions of platelet behavior.

\subsection{Negative Prestress}

In the absence of prestress $\left(T_{\mathrm{o}}\right)$, the mathematical model produced preliminary solutions that generated significant numerical error $(\varepsilon)$, thereby failing to preserve platelet volume (Section 4.4.1). To rationalize this behavior, it is generally accepted that cellular prestress is defined by the cytoskeletal components, and is distinguished by the isometric tension generated, in the majority, by the acto-myosin contractile components of the complex actin network. Interestingly, the resultant magnitude of membrane prestress suggests that the resting platelet membrane in particular, is under compression. One potential explanation of this outcome would be the role of the microtubule coil, which lies along the circumference of the platelet, and has a notable influence on controlling shape stability and overall deformability (66). This inherent compression may also prevent the pre-loaded membrane from a natural tendency to separate from its intracellular components. Considering the tensegrity (or tensional integrity) model, which describes the mechanical properties of cellular structure, this theory supposes that the intracellular structure of the cell consists of components under tension balanced by those under compression (68). Applied to the platelet structure, evidence suggests that cellular stability is ultimately upheld by tension elements that are composed of actin microfilaments, while microtubules act as compression elements (119). 
In terms of general model implications, then, the incorporation of a prestress component infers the significant contributions of these cytoskeletal components, but fails to directly model the mechanical behavior of this complex network of proteins (i.e. microfilaments, microtubules, actin filaments). The current model, then, is committed to a continuum mechanics approach that characterizes the global mechanical properties of the platelet membrane, but indirectly acknowledges a heterogeneous cell structure maintained by an underlying cytoskeleton (118).

\subsection{Clinical Relevance}

Despite the large body of platelet research available, daily issues of thrombosis in clinical settings are a testament to what is still unknown. Manufacturers strive to improve the design of MHVs to minimize thromboembolic events in patients (Section 2.2.2), but the recurrence of such problems points to holes in our core understanding of the phenomenon, as does the wide range of medications and dosages administered to curtail thrombosis in these patients. Transfusion and human blood storage techniques are other activities where thrombosis is a constant threat and present another source of motivation for developing a better understanding of the fundamental causes of this process.

In particular, with the advent of refined techniques for probing the mechanical behavior of the platelet membrane, one may hope in future investigations to construct a more accurate representation of the local forces necessary to indirectly activate platelets (via cell lysis) through shear stress, either at once, or after multiple repetitions as experienced by circulating platelets. Then, for example, the design of MHVs could be evaluated for their ability to keep such forces to a minimum, even before the valve is tested in vivo, as monitoring single platelets in the blood flow is becoming possible in computer simulations $(120)$.

Essentially, the current work contributes to this clinically relevant pursuit by establishing a validated constitutive model that predicts platelet membrane responses (i.e. membrane tension, stretch) to an applied force. In terms of loading limits, the model can also probe the strength of the platelet membrane, defining the behavioral profile that fails to preserve the structural and functional integrity (i.e. cell lysis) of a single platelet. 


\subsection{Limitations of the Current Work and Future Recommendations}

Although the implementation of deformation data sets from two platelet species proved advantageous in their capacity to validate the model, a more representative mechanical description of the human platelet membrane is required. Specifically, the model lacks a definitive and robust set of deformation data and qualitative descriptions (i.e. signs of activation, platelet lysis) for average human platelet volumes, which would significantly improve the accuracy of membrane material constants. To address these deficiencies, one must conserve the general methodologies described for murine platelet sample preparations and platelet imaging to obtain a large inactive platelet population that follows a normal distribution of volumes. Model development also presumes consistencies in the general material behavior between species, imposing a hyperelastic material concept. As such, the current work implemented aspiration data of human platelets that mainly concentrated on the magnitude of platelet deformation to imposed pressure, without considerations to the time history of platelet tongue deformation $(86,87,88,11)$. Ultimately, a failure to recognize potential viscoelastic properties in the material model concept would result in a misrepresentative model of human platelet deformation. In real-time, future investigations of human membrane responses to micropipette aspiration must definitively resolve viscoelastic uncertainties, quantifying the changes in platelet tongue length $\left(L_{\mathrm{p}}\right)$ as a function of time. Although insignificant to current model evaluations of membrane strength, strain rate may prove significant in future attempts to model platelet membrane mechanical behavior.

As mentioned, the current model provides a simplistic explanation of platelet deformation, as it characterizes the global membrane mechanical properties without considering the significant structural contributions of cytoskeletal components. As this complex network of proteins influences membrane deformability (66), a more complex model might also provide insight into how mechanical manipulation of the membrane mediates biological pathways (linking mechanics to microstructure and molecular biochemistry) (119). To verify the mechanical contributions of these structural components to the platelet membrane, as characterized by resultant material constants and descriptions of tension and stretch, future work could follow the processing practices detailed in a study by White et al. (66). Specifically, platelet populations in this study (66) were independently treated with anti-microtubule agents (vincristine, colchicines) or actin microfilaments 
assembly antagonists (Cytochalasin B) to verify the cytoskeletal impact on platelet membrane deformability.

In summary, the current constitutive model offers an effective mechanism to characterize the mechanical properties of the platelet membrane in response to micropipette aspiration. Constitutive modeling of the platelet membrane, however, cannot be summarized by one form of mechanical manipulation alone (100). To gain a more comprehensive description of the membranes behavioral versatility, then, novel constitutive modeling methods employing alternative manipulation techniques are necessary. 


\section{Chapter 6}

\section{Conclusions}

The overall goal of the current work was to model the mechanical behavior of platelets, which play an integral role in circulatory health and proved to be a challenging endeavour that demanded precision and skill in both experimental and mathematical applications. To date, efforts to characterize the mechanical behavior of such intrinsically sensitive and thrombogenic blood components is sparse, thereby motivating this research towards a novel and valuable product. In reviewing literature, the role of mechanical stimuli in hemostasis and pathological thrombosis is such that one must develop a multidisciplinary concept of blood coagulation to establish a comprehensive description of clotting agonists. At the cellular level, techniques to investigate such biomechanical behaviours range from whole blood simulations of variable flow conditions to those limited to unicellular manipulations. A necessary extension of single platelet manipulations requires a more general evaluation of platelet membrane mechanical behavior, as most investigations were centered on properties of cytoskeletal function $(65,66)$ and glycoprotein interaction $(78,79)$. Addressing these limitations, micropipette aspiration is a technique that offers a real-time and visually simple perspective of membrane deformation that proves highly useful in its capacity to control membrane behavior.

To the advantage of mathematical model validations, quantitative information extracted from aspiration data sets proves invaluable to theoretical development. Further, in an integrated effort to accurately describe the average membrane response (i.e. stress, strain) to mechanical manipulation, the current work develops a constitutive equation with applicability to both platelet species. Ultimately, by generating an optimized set of membrane material constants the model maintains precision in simulating micropipette aspiration, and accurately predicts membrane mechanical responses to this deformation mechanism. Although lacking evidence of mechanotransduction in murine platelet manipulations, qualitative insight confirming the occurrence of platelet lysis establishes future opportunities to confidently define membrane strength. Clearly, much work remains, not only on the mechanistic and biochemical aspects, but also on the integration of all the 
pieces of information, before a comprehensive picture can be drawn. Also, to improve clinical relevance a natural complement to this work would therefore demand a modernized and robust biomechanical investigation of human platelets, defining model significance as it relates to vasculature and prosthetic devices. Ultimately, cumulative information regarding platelet activation and lysis, deformation and material properties amounts to a more thorough understanding of platelets' contributions to physiological thrombosis. Better ways to prevent or reduce thrombosis should be natural dividends from such a collective effort. 


\section{Appendix A}

Standard Operating Procedure for Rodent Anaesthetization.

\begin{tabular}{|c|c|c|c|}
\hline \multicolumn{4}{|c|}{$\begin{array}{c}\text { National Research Council } \\
\text { Institute for Biological Sciences } \\
\text { Standard Operating Procedure (SOP) }\end{array}$} \\
\hline \multicolumn{4}{|c|}{$\begin{array}{l}\text { TITLE/SUBJECT: } \\
\text { RODENT INHALANT ANESTHESIA }\end{array}$} \\
\hline \multicolumn{2}{|l|}{ SOP NO.: } & \multicolumn{2}{|c|}{$\begin{array}{r}\text { COPMPUTER FILE NO.: } \\
\text { AR06003 }\end{array}$} \\
\hline \multicolumn{4}{|c|}{ ORIGINATING GROUP: } \\
\hline $\begin{array}{l}\text { STATUS: } \\
\text { Final, March 8, } 2007\end{array}$ & $\begin{array}{r}\text { VERSION: } \\
2 \\
\end{array}$ & $\begin{array}{c}\text { EFFECTIVE DATE: } \\
\text { March 8, } 2007 \\
\end{array}$ & $\begin{aligned} & \text { PAGES: } \\
& 1 \text { of } 4 \\
&\end{aligned}$ \\
\hline
\end{tabular}




\section{Introduction}

1.1 Anesthetizing laboratory animals is often a key component of many experimental protocols. It is therefore critical that the appropriate agent(s) be chosen and that it be administered properly in order to address humane issues and ultimately to be able to obtain valid research data.

1.2 Inhalant anesthetic agents allow for rapid induction and recovery. However, due to their high volatility they usually need to be administered by a precision vaporizer.

1.3 Inhalants also offer the advantage of causing minimal chemical contamination to the subject. The percentage of agent taken up and recovered as metabolites is $0.17 \%$ for isoflurane and $20 \%$ for halothane. It is for this reason that isoflurane is considered safer for personnel who are exposed to the waste gases.

1.4 In terms of animal preference, it appears that halothane, with its somewhat sweet odor, is preferable to the more pungent smelling isoflurane.

\section{Purpose}

2.1 The purpose of this SOP is to provide guidelines to those persons anesthetizing rats and mice in order that it is performed in a safe and efficient manner.

\section{Scope}

3.1 These guidelines apply to all research animals used in the IBS. However, it should be emphasized that each protocol and animal strain has unique differences that could be adversely affected by the anesthetic regime and as such each protocol should assessed on an individual basis.

\section{Pre-Anesthetic Preparation of the Animal}

4.1 Animals shipped from outside suppliers should be acclimatized for a minimum of 5 days prior to being anesthetized.

4.2 Animals should be examined to confirm they are clinically healthy.

4.3 Animals should be weighed and the weights recorded. This information is important for accurately determining drug dosages and as a baseline for health monitoring.

4.4 Fasting, unless required by the protocol, is not normally required since rodents can not regurgitate. Their high metabolic rate also makes them susceptible to hypoglycemia if fasted. Thirdly, rodents are coprophagic, so it is actually very difficult to prevent them from eating prior to surgery. Restricting water is not advisable at any time.

4.5 Pre-anesthetic sedation is not generally required in the rat or mouse.

\section{Anesthesia Induction and Maintenance Procedures}

5.1 Place animal in anesthetic chamber.

5.2 Turn oxygen flow meter to $2 \mathrm{~L} /$ minute.

5.3 Turn anesthetic vaporizer to $4 \%$.

5.4 After 1-5 minutes (length of time is $\propto$ to size of container) or as soon as the animal becomes recumbent reduce the vaporizer setting to a maintenance level (see chart). 
5.5 At a maintenance level of anesthetic the animal can be;

i) maintained for an extended period

ii) removed from the chamber and then hooked up to a facemask.

iii) removed from chamber and allowed to recover, usually within a few minutes.

6. Chart

\begin{tabular}{|c|c|c|}
\hline Anesthetic Agent & Induction & Maintenance \\
\hline \multirow{2}{*}{$\begin{array}{c}\text { Isoflurane } \\
\text { (Aerrane } \AA \text {, Forane) }\end{array}$} & $\begin{array}{c}\text { Anesthetic Concentration: } \\
4 \% \\
(1-3 \text { minutes })\end{array}$ & $\begin{array}{l}\text { Anesthetic Concentration: } \\
\qquad 1.5-3 \%\end{array}$ \\
\hline & $\begin{array}{c}\text { Oxygen Flow Rate }(\mathrm{L} / \mathrm{min}) \\
2 \mathrm{~L} / \text { minute }\end{array}$ & $\begin{array}{c}\text { Oxygen Flow Rate (L/min) } \\
1-1.5 \mathrm{~L} / \text { minute }\end{array}$ \\
\hline \multirow[t]{2}{*}{$\begin{array}{l}\text { Halothane } \\
\text { (Fluothane } \mathbb{B} \text { ) }\end{array}$} & $\begin{array}{c}\text { Anesthetic Concentration: } \\
4 \% \\
(1-3 \text { minutes })\end{array}$ & $\begin{array}{c}\text { Anesthetic Concentration: } \\
\qquad 1-2 \%\end{array}$ \\
\hline & $\begin{array}{c}\text { Oxygen Flow Rate (L/min) } \\
2 \mathrm{~L} / \text { minute }\end{array}$ & $\begin{array}{c}\text { Oxygen Flow Rate }(\mathrm{L} / \mathrm{min}) \\
\text { L/minute }\end{array}$ \\
\hline
\end{tabular}

\section{Animal Care During Anesthesia}

6.1 Sterile ophthalmic ointment should be placed in the eyes of all anesthetized rodents. Rodents are normally exophthalmic and most anesthetics oblate the blink reflex making them susceptible to corneal desiccation.

6.2 Animals anesthetized for periods greater than 30 minutes should be provided with a supplemental heating device, preferably a circulating water-heating pad. When possible core body temperatures should be monitored.

\section{Post-Anesthetic Care}

7.1 Animals having undergone long periods of anesthesia should be administered warm $\left(37-38{ }^{\circ} \mathrm{C}\right)$ sterile saline (SC or IP) at the dose of $10 \mathrm{ml} / \mathrm{kg} /$ hour of anesthesia.

7.2 All animals should be observed until they have recovered before returning them to their normal room/cubicle. Recovery is defined as being able to perform purposeful movements and being conscious of their surroundings.

7.3 Animals should be housed individually or housed with other animals having undergone the same procedure for at least 24 hours after surgery.

7.4 All animals undergoing painful procedures should be administered analgesic drugs. See SOP on rodent analgesia for further details. Analgesia will not be administered if its omission is explained in the in the ACC-approved protocol 


\section{Miscellaneous Comments}

8.1 Oxygen flow rates in an open anesthetic setup should be three times the minute volume of the animal being anesthetized. The minute volume of a rat is approximately $75 \mathrm{ml} / \mathrm{minute}$. Therefore a minimum oxygen flow rate of $0.225 \mathrm{~L}$ /minute is required. However, high oxygen flow rates are generally required because current anesthetic vaporizers are considered inaccurate at flow rates of less than 0.7 $\mathrm{L} /$ minute.

8.2 Oxygen flow rates in excess of those recommended are wasteful and potentially harmful to those exposed to the waste gases.

8.3 The administration of anesthetic concentrations in excess to those recommended or for periods longer than those recommended may put the animal at risk of dying.

8.4 Do not hesitate to contact Animal Resources' personnel in order to obtain additional information or assistance with any aspect of anesthetizing laboratory animals. 


\section{Appendix B}

\section{Deformed Platelet Assumptions Overview}

Zone 1: sphere of radius $\boldsymbol{r}_{\mathrm{s}}$

$r=r_{s} \sin \phi ; \phi_{\mathrm{s}} \leq \phi \leq \pi ; \phi_{s}=\arcsin \left(\frac{R_{p}}{r_{s}}\right)$

$z=r_{s}(1+\cos \phi) ; \mathrm{dr}=\cos \phi \mathrm{ds} ; d z=-\sin \phi d s ; \mathrm{ds}=\mathrm{r}_{\mathrm{s}} \mathrm{d} \phi$

where $r_{\mathrm{s}}=r_{\mathrm{n}}=r_{\theta}$,

$\mathrm{T}_{\mathrm{s}}{ }^{1}=\left(\frac{p-p_{o}}{2}\right) r_{s}=T_{\theta}{ }^{1}$, consequence of the spherical geometry (79)

$\lambda_{s}=\frac{r_{s}}{\sqrt{R_{s}^{2} \cos ^{2} \Phi+S_{s}^{2} \sin ^{2} \Phi}} \frac{d \phi}{d \Phi} ; \lambda_{\theta}=\frac{r_{s} \sin \phi}{R_{s} \sin \Phi}$

\section{Zone 2: cylinder of radius $\boldsymbol{R}_{\mathrm{p}}$}

$r=R_{p} ; \phi=\frac{\pi}{2}$

$r_{s}\left(1+\cos \phi_{s}\right) \leq z \leq r_{s}\left(1+\cos \phi_{s}\right)+L_{p}-R_{p} ; d r=0 ; d z=d s ; \mathrm{d} \phi=0$

where $r_{n}=\infty ; r_{\theta}=R_{p}$

$T_{s}^{2}=\left(\frac{p-p_{o}}{2}\right) r_{s}=\left(\frac{p-p_{i}}{2}\right) R_{p}=T_{s}^{1}=T_{s}^{3}$ therefore $p=\frac{p_{\mathrm{o}} r_{s}-p_{i} R_{p}}{r_{s}-R_{p}}$

$T_{\theta}^{2}=p R_{p}:$ circumferential tension is assumed by the pipette material,

$\therefore$ no role in the characterization of the platelet membrane material properties

$\lambda_{s}=\frac{1}{\sqrt{R_{s}^{2} \cos ^{2} \Phi+S_{s}^{2} \sin ^{2} \Phi}} \frac{d z}{d \Phi} ; \lambda_{\theta}=\frac{R_{p}}{R_{s} \sin \Phi}$

\section{Zone 3: hemisphere of radius $R p$}

$r=R_{p} \sin \phi ; 0 \leq \phi \leq \frac{\pi}{2}$

$z=r_{s}\left(1+\cos \phi_{s}\right)+L_{p}-R_{p}(1-\cos \phi) ; d r=\cos \phi d s ; \mathrm{d} \phi=-\sin \phi d s ; d s=R_{\mathrm{p}} \mathrm{d} \phi$

where $r_{n}=r_{\theta}=R_{p}$; the volume of the hemispherical cap is dependent on $R_{\mathrm{p}}$

$$
\begin{aligned}
& T_{s}^{3}=\left(\frac{p-p_{i}}{2}\right) R_{p}=T_{\theta}{ }^{3} \\
& \lambda_{s}=\frac{R_{p}}{\sqrt{R_{s}{ }^{2} \cos ^{2} \Phi+S_{s}{ }^{2} \sin ^{2} \Phi}} \frac{d \phi}{d \Phi} ; \lambda_{\theta}=\frac{R_{p} \sin \phi}{R_{s} \sin \Phi}
\end{aligned}
$$




\section{Appendix C}

\section{Undeformed and Deformed Membrane Elements in Curvilinear Coordinates}

Platelet shape is based on a similar axisymmetric geometry outlined by Evans (98), where the platelet geometry is also defined by curvilinear coordinates $(S, \Phi, \Theta)$ and $(s, \phi, \theta)$ in the undeformed and deformed contours, respectively. The distance along the contour of the axisymmetric shell is defined by $s$ (or $S$ ), the coordinate $\phi($ or $\Phi)$ defines the angle between the $z$-axis and the outward normal $(n)$ to the shell surface, and the coordinate $\theta$ (or $\Theta$ ) is the azimuthal angle (84). An application of this coordinate system that defines undeformed and deformed surface elements of a platelet is illustrated in Figure C.1.

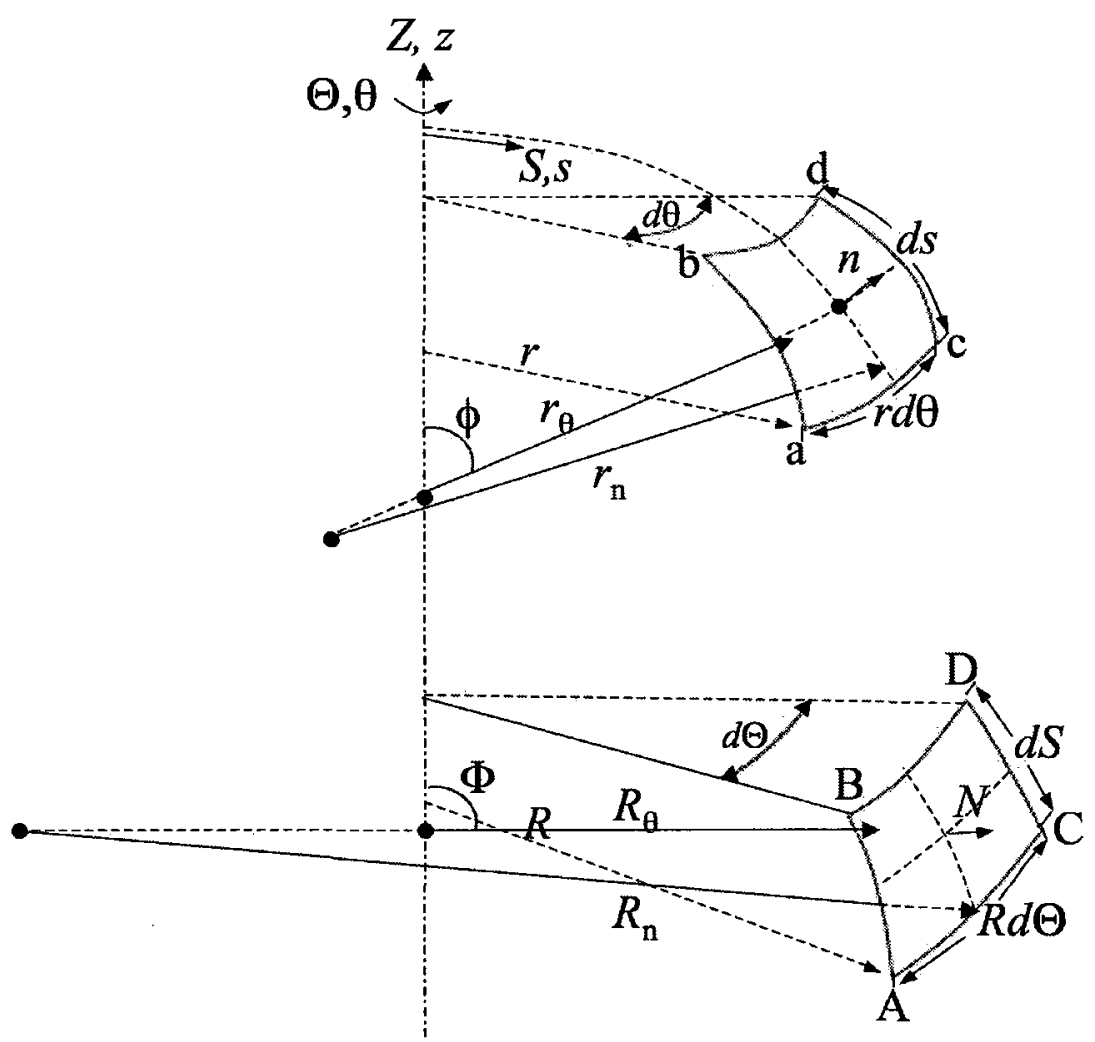

Figure C.1: Graphical representation of the initial (undeformed) and final (deformed) geometries of an element on the axisymmetric membrane surface. The spatial limitations of this element are labeled A to D in the undeformed orientation, and a to $\mathrm{d}$ after element deformation. Spatial coordinates for this element are $(R, \Theta, Z)$ and $(r, \theta, z)$ for the initial and final states, respectively; the curvilinear coordinates for this element are $(S, \Theta)$ and $(s, \theta)$. $\left(R_{\mathrm{n}}, R_{\theta}\right)$ and $\left(r_{\mathrm{n}}, r_{\theta}\right)$ are principal radii of curvature at points $(S, \Theta)$ and $(s, \theta)$ on the undeformed and deformed platelet surface elements, respectively. $r_{n}$ (or $\left.R_{n}\right)$ defines a local arc of the surface meridian. $r_{\theta}$ (or $R_{\theta}$ ) defines a local arc which is parallel to the shell normal and is orthogonal to the meridianal contour. [Adapted from (84)]. 


\section{Appendix D}

\section{Proof of Equilibrium Equations}

The general loading of an element on the shell surface results in membrane forces and their differential changes that are depicted below. Using the differential area along the deformed platelet contour, $(d A=r d \theta d s)$, 


$$
\begin{aligned}
& \frac{\partial\left(N_{s} r \mathrm{e}_{s}\right)}{\partial s} d \theta d s+\frac{\partial\left(N_{s \theta} r \mathrm{e}_{\theta}\right)}{\partial s} d \theta d s+\frac{\partial\left(N_{\theta} \mathrm{e}_{\theta}\right)}{\partial \theta} d \theta d s+\frac{\partial\left(N_{\theta s} \mathrm{e}_{s}\right)}{\partial \theta} d s d \theta+r p_{s} \mathrm{e}_{s} d \theta d s+\ldots \\
& +r p_{\theta} \mathrm{e}_{\theta} d \theta d s+r p_{n} \mathrm{e}_{n} d \theta d s=0
\end{aligned}
$$

Expanding the first four terms :

$$
\begin{aligned}
& \frac{\partial N_{s}}{\partial s} r \mathrm{e}_{\mathrm{s}}+N_{s} \frac{\partial r}{\partial s} \mathrm{e}_{s}+N_{s} r\left(\frac{\partial \mathrm{e}_{\mathrm{s}}}{\partial s}\right)+\frac{\partial N_{s \theta}}{\partial s} r \mathrm{e}_{\theta}+N_{s \theta} \frac{\partial r}{\partial s} \mathrm{e}_{\theta}+N_{s \theta} r\left(\frac{\partial \mathrm{e}_{\theta}}{\partial s}\right)+\left(\frac{\partial N_{\theta}}{\partial \theta}\right) \mathrm{e}_{\theta}+\ldots \\
& +N_{\theta}\left(\frac{\partial \mathrm{e}_{\theta}}{\partial \theta}\right)+\left(\frac{\partial N_{\theta s}}{\partial \theta}\right) \mathrm{e}_{s}+N_{\theta s}\left(\frac{\partial \mathrm{e}_{s}}{\partial \theta}\right)+r p_{s} \mathrm{e}_{\mathrm{s}}+r p_{\theta} \mathrm{e}_{\theta}+r p_{n} \mathrm{e}_{\mathrm{n}}=0
\end{aligned}
$$

Using derivatives of unit vectors $e_{s}$ and $e_{\theta}(109)$ :

$$
\begin{aligned}
& \frac{\partial \mathrm{e}_{\mathrm{s}}}{\partial s}=-\frac{\mathrm{e}_{\mathrm{n}}}{r_{n}} ; \frac{\partial \mathrm{e}_{\theta}}{\partial s}=0 ; \frac{\partial \mathrm{e}_{\theta}}{\partial \theta}=-\mathrm{e}_{s} \cos \phi-\mathrm{e}_{\mathrm{n}} \sin \phi ; \frac{\partial \mathrm{e}_{s}}{\partial \theta}=\mathrm{e}_{\theta} \cos \phi \\
& \frac{\partial N_{s}}{\partial s} r \mathrm{e}_{s}+N_{s} \frac{\partial r}{\partial s} \mathrm{e}_{s}+N_{s} r\left(-\frac{\mathrm{e}_{\mathrm{n}}}{r_{n}}\right)+\frac{\partial N_{s \theta}}{\partial s} r \mathrm{e}_{\theta}+N_{s \theta} \frac{\partial r}{\partial s} \mathrm{e}_{\theta}+N_{s \theta} r(0)+\left(\frac{\partial N_{\theta}}{\partial \theta}\right) \mathrm{e}_{\theta}+\ldots \\
& +N_{\theta}\left(-\mathrm{e}_{s} \cos \phi-\mathrm{e}_{\mathrm{n}} \sin \phi\right)+\left(\frac{\partial N_{\theta s}}{\partial \theta}\right) \mathrm{e}_{s}+N_{\theta s}\left(\mathrm{e}_{\theta} \cos \phi\right)+r p_{s} \mathrm{e}_{s}+r p_{\theta} \mathrm{e}_{\theta}+r p_{n} \mathrm{e}_{\mathrm{n}}=0
\end{aligned}
$$

Using $\frac{d r}{d s}=\cos \phi$ and rearranging :

$$
\begin{aligned}
& \mathrm{e}_{\mathrm{n}}\left(-N_{\theta} \sin \phi-N_{s} \frac{r}{r_{n}}+r p_{n}\right)+\mathrm{e}_{\theta}\left(\frac{\partial N_{\theta}}{\partial \theta}+N_{s \theta} \cos \phi+r \frac{\partial N_{s \theta}}{\partial s}+N_{\theta s} \cos \phi+r p_{\theta}\right)+\ldots \\
& +\mathrm{e}_{s}\left(r \frac{\partial N_{s}}{\partial s}+N_{s} \cos \phi+\frac{\partial N_{\theta s}}{\partial \theta}-N_{\theta} \cos \phi+r p_{s}\right)=0 .
\end{aligned}
$$

The equilibrium equations defining $\mathrm{N}_{\mathrm{s}}, \mathrm{N}_{\theta}, \mathrm{N}_{s \theta}$ and $\mathrm{N}_{\theta s}$ are therefore :

$$
\begin{aligned}
& \sum F_{n}=-N_{\theta} \sin \phi-N_{s} \frac{r}{r_{n}}+r p_{n}=0 \\
& \sum F_{\theta}=\frac{\partial N_{\theta}}{\partial \theta}+N_{s \theta} \cos \phi+r \frac{\partial N_{s \theta}}{\partial s}+N_{\theta s} \cos \phi+r p_{\theta}=0 \\
& \sum F_{s}=r \frac{\partial N_{s}}{\partial s}+\left(N_{s}-N_{\theta}\right) \cos \phi+\frac{\partial N_{\theta s}}{\partial \theta}+r p_{s}=0
\end{aligned}
$$

using $d s=r_{n} d \phi \quad$ and $\quad \sin \phi=\frac{r}{r_{\theta}}$

$-N_{\theta} \sin \phi-N_{s} \frac{r}{r_{n}}+r p_{n}=0$

$-N_{\theta} \frac{r}{r_{\theta}}-N_{s} \frac{r}{r_{n}}+r p_{n}=0$

$\frac{N_{\theta}}{r_{\theta}}+\frac{N_{s}}{r_{n}}=p_{n}$ 


$$
\begin{aligned}
& \frac{\partial N_{\theta}}{\partial \theta}+N_{s \theta} \cos \phi+r \frac{\partial N_{s \theta}}{\partial s}+N_{\theta s} \cos \phi+r p_{\theta}=0 \\
& \frac{1}{r} \frac{\partial N_{\theta}}{\partial \theta}+\frac{N_{s \theta} \cos \phi}{r}+\frac{1}{r_{n}} \frac{\partial N_{s \theta}}{\partial \phi}+\frac{N_{\theta s} \cos \phi}{r}+p_{\theta}=0
\end{aligned}
$$

$r \frac{\partial N_{s}}{\partial s}+\left(N_{s}-N_{\theta}\right) \cos \phi+\frac{\partial N_{s \theta}}{\partial \theta}+r p_{s}=0$

$\frac{1}{r_{n}} \frac{\partial N_{s}}{\partial \phi}+\frac{\left(N_{s}-N_{\theta}\right) \cos \phi}{r}+\frac{1}{r} \frac{\partial N_{\theta s}}{\partial \theta}+p_{s}=0$

since $N_{\theta}=T_{\theta}, N_{s}=T_{s}, N_{s \theta}=N_{\theta s}=T_{s \theta}=T_{\theta s}, p_{\theta}=0, \quad$ C. 5 - C. 7 can be expressed as,

$\frac{T_{\theta}}{r_{\theta}}+\frac{T_{s}}{r_{n}}=p_{n}$

$\frac{1}{r} \frac{\partial T_{\theta}}{\partial \theta}=0$

$\frac{\partial T_{\theta}}{\partial \theta}=0$

$\frac{1}{r_{n}} \frac{\partial T_{s}}{\partial \phi}+\frac{\left(T_{s}-T_{\theta}\right) \cos \phi}{r}+p_{s}=0,\left(\right.$ where $\left.p_{s}=0\right)$

Finally, these equilibrium equations can be expressed as (92),

$$
\mathrm{T}_{\mathrm{s}}=\frac{P}{2 K_{2}} \quad \mathrm{~T}_{\theta}=\frac{\left(2 K_{2}-K_{1}\right)}{2 K_{2}^{2}} P
$$

assuming $\mathrm{K}_{1}=r_{n}^{-1}, \mathrm{~K}_{2}=r_{\theta}^{-1}, \mathrm{P}=\mathrm{p}_{\mathrm{n}}$

$\mathrm{T}_{\mathrm{s}}=\frac{p_{n}}{2} \mathrm{r}_{\theta} \quad \mathrm{T}_{\theta}=p_{n} \mathrm{r}_{\theta} \frac{\left(1-r_{\theta}\right)}{2 r_{n}}$ 


\section{Appendix E}

\section{Constitutive Description of Platelet Membrane}

$$
\begin{aligned}
& W=-\frac{c_{1}}{2} \ln (1-Q) \quad \text { with } Q=c_{2} I_{2}^{n_{2}}+c_{3} I_{2}^{n_{3}} \text { and } I_{2}=\lambda_{s}^{2} \lambda_{\theta}^{2}-1=\left(\frac{d A}{d A_{o}}\right)^{2}-1 \\
& \frac{\partial Q}{\partial I_{2}}=Q^{\prime}=c_{2} n_{2} I_{2}^{\left(n_{2}-1\right)}+c_{3} n_{3} I_{2}^{\left(n_{3}-1\right)} ; \quad \frac{\partial W}{\partial I_{2}}=\frac{c_{1}}{2} \frac{Q^{\prime}}{(1-Q)} \\
& T_{s}=T_{\theta}=2 \lambda_{s} \lambda_{\theta} \frac{\partial W}{\partial I_{2}} \\
& T_{s}=T_{\theta}=c_{1} \lambda_{s} \lambda_{\theta}\left\{\frac{c_{2} n_{2}\left(\lambda_{s}^{2} \lambda_{\theta}^{2}-1\right)^{\left(n_{2}-1\right)}+c_{3} n_{3}\left(\lambda_{s}^{2} \lambda_{\theta}^{2}-1\right)^{\left(n_{3}-1\right)}}{1-c_{2}\left(\lambda_{s}^{2} \lambda_{\theta}^{2}-1\right)^{n_{2}}-c_{3}\left(\lambda_{s}^{2} \lambda_{\theta}^{2}-1\right)^{n_{3}}}\right\}
\end{aligned}
$$




\section{Appendix F}

\section{Zone-dependent Descriptions of Membrane Tension}

\section{Zone 1:}

At the bottom pole, $(\Phi=\phi=\pi)$ :

$$
\begin{aligned}
\lambda_{\mathrm{s}} & =\lambda_{\theta} \\
\frac{r_{s}}{R_{s}}\left(\frac{d \phi}{d \Phi}\right)_{\pi} & =\frac{r_{s}}{R_{s}}\left(\frac{\sin \phi}{\sin \Phi}\right)_{\pi},
\end{aligned}
$$

therefore,

$$
\left(\mathrm{p}-\mathrm{p}_{0}\right) r_{s}-2 c_{1}\left(\frac{r_{s}}{R_{s}}\right)^{2}\left(\frac{d \phi}{d \Phi}\right)_{\pi}^{2}\left\{\frac{c_{2} n_{2}\left[\left(\frac{r_{s}}{R_{s}}\right)^{4}\left(\frac{d \phi}{d \Phi}\right)_{\pi}^{4}-1\right]^{\left(n_{2}-1\right)}+c_{3} n_{3}\left[\left(\frac{r_{s}}{R_{s}}\right)^{4}\left(\frac{d \phi}{d \Phi}\right)_{\pi}^{4}-1\right]^{\left(n_{3}-1\right)}}{1-c_{2}\left[\left(\frac{r_{s}}{R_{s}}\right)^{4}\left(\frac{d \phi}{d \Phi}\right)_{\pi}^{4}-1\right]^{n_{2}}-c_{3}\left[\left(\frac{r_{s}}{R_{s}}\right)^{4}\left(\frac{d \phi}{d \Phi}\right)_{\pi}^{4}-1\right]^{n_{3}}}\right\}-2 T_{o}=0
$$

Zone 3:

At the bottom pole, $(\Phi=\phi=0)$ :

$$
\begin{aligned}
& \lambda_{\mathrm{s}}=\lambda_{\theta} \\
& \frac{R_{p}}{R_{s}}\left(\frac{d \phi}{d \Phi}\right)_{0}=\frac{R_{p}}{R_{s}}\left(\frac{\sin \phi}{\sin \Phi}\right)_{0},
\end{aligned}
$$

therefore,

$$
\left(\mathrm{p}-\mathrm{p}_{\mathrm{i}}\right) R_{p}-2 c_{1}\left(\frac{R_{p}}{R_{s}}\right)^{2}\left(\frac{d \phi}{d \Phi}\right)_{0}^{2}\left\{\frac{c_{2} n_{2}\left[\left(\frac{R_{p}}{R_{s}}\right)^{4}\left(\frac{d \phi}{d \Phi}\right)_{0}^{4}-1\right]^{\left(n_{2}-1\right)}+c_{3} n_{3}\left[\left(\frac{R_{p}}{R_{s}}\right)^{4}\left(\frac{d \phi}{d \Phi}\right)_{0}^{4}-1\right]^{\left(n_{3}-1\right)}}{1-c_{2}\left[\left(\frac{R_{p}}{R_{s}}\right)^{4}\left(\frac{d \phi}{d \Phi}\right)_{0}^{4}-1\right]^{n_{2}}-c_{3}\left[\left(\frac{R_{p}}{R_{s}}\right)^{4}\left(\frac{d \phi}{d \Phi}\right)_{0}^{4}-1\right]^{n_{3}}}\right\}-2 T_{o}=0
$$




\section{Appendix G}

\section{MATLAB M-files: Commented}

\section{1-Material Constants Identification/main:}

clc

clear

format long

$\%$ Declare Global Variables

global rp rrs ss lpexp ppi ppo pp rs n2 n3

species = 'human'; $\quad \%$ implement 'human' or 'mouse' deformation data

shaperatio $=0.25 ; \quad \%$ shaperatio is $\mathrm{ss} / \mathrm{rrs}$, and is between 0.15 and 0.25 , based on info from human platelets

$\%$ Read in raw experimental data from rawdata .txt switch species case 'human' case 'mouse'

fid = fopen('rawdatahuman.txt', 'rt');

$$
\text { fid = fopen('rawdatamouse.txt', 'rt'); }
$$

end

$\mathrm{c}=$ textscan (fid, $\left.{ }^{1} \% \mathrm{f} \% \mathrm{f} \% \mathrm{f}, 1\right)$;

$\mathrm{rp}=\mathrm{c}\{1\}$;

$\mathrm{v} 0=\mathrm{c}\{2\}$;

$\mathrm{c}=$ textscan(fid, $\left.{ }^{\circ} \% \mathrm{f} \% \mathrm{f}^{\prime}\right)$;

fclose(fid);

deltap $=\mathrm{c}\{1\}$;

deltap $=$ deltap .98 .067

$\mathrm{Ip}=\mathrm{c}\{2\}$;

$\%$ Synthesize $\mathrm{n}$ data points

$\mathrm{n}=12$;

$\mathbf{t}=1: 1$ :length(deltap);

spdeltap $=$ spline $(t$,deltap $)$;

splp $=$ spline $(t, l p)$

$\mathfrak{t t}=$ linspace $(1$, length $($ deltap $), n)$

deltapexp = ppval $($ spdeltap,, $\mathrm{tt})$

deltapexp = deltapexp'; \%reads and converts ( 2 sig. digits) from text file 'fid' into a 3 cell array ' $c$ ', format is used 1 time

$\%$ pipette radius $(\mathrm{m})$

$\%$ volume of intact platelet $\left(\mathrm{m}^{3}\right)$

$\% \%$ reads and converts ( 2 sig. digits) from text file 'fid' into a

2 cell array 'c'

$\%$ closes text file 'fid'

$\%$ aspiration pressure $\left(\mathrm{cmH}_{2} \mathrm{O}\right)$

$\%$ aspiration pressure $(\mathrm{Pa})$

$\%$ aspiration length $(\mathrm{m})$

$\%$ end using rawdata . $\mathrm{txt}$

$\%$ vector with identical length to vector 'deltap' $\%$ produces piecewise polynomials of the cubic spline interpolant to the aspiration pressure values of 'deltap' at the data sites of the vector ' $t$ '

$\%$ produces piecewise polynomials of the cubic spline interpolant to the data values of 'Ip' at the data sites of the vector ' $t$ '

\%creates a linearly spaced vector with 12 points between 1 and the length of vector 'deltap'

$\%$ synthesized experimental aspiration pressure $(\mathrm{Pa})$ values from the piecewise polynomial 'spdeltap' at the linearly spaced entries defined by the vector ' $t \mathrm{t}$ ' $\%$ forms transpose of vector 'deltapexp' 
$\operatorname{lpexp}=\operatorname{ppval}($ splp,tt $)$

lpexp = lpexp';

ppo $=101300 *$ ones(length $($ lpexp $), 1)$

ppi $=$ ppo - deltapexp;

rrs $=\left(3^{*} \mathrm{v} 0 /\left(4^{*} \mathrm{pi}^{*} \text { shaperatio }\right)\right)^{\wedge}(1 / 3)$

ss $=$ shaperatio* ${ }^{*}$ rs;

rs=zeros(length(lpexp),1);

for $\mathrm{i}=1:$ length(lpexp)

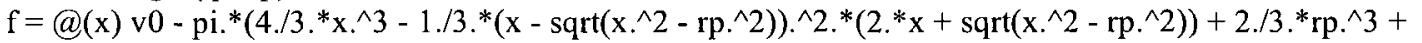

$\left(\right.$ lpexp(i) - rp).* ${ }^{*}$ rp. $\left.{ }^{\wedge}\right)$
$\%$ synthesized experimental aspiration length $(\mathrm{m})$ values from the piecewise polynomial 'splp' at the linearly spaced entries defined by the vector ' $t$ '

$\%$ forms transpose of vector 'deltapexp'

$\%$ determines atmospheric pressure $(\mathrm{Pa})$

$\%$ intracellular pressure

$\%$ determines the undeformed major radius $\left(\mathrm{R}_{\mathrm{s}}\right)$ of the platelet

$\%$ determines the undeformed minor radius $\left(\mathrm{S}_{\mathrm{s}}\right)$ of the platelet $\%$ defines the deformed spherical radius 'rs' as a matrix of zeros that is the length of vector 'lpexp' by 1 in size $\%$ defines a function ' $\mathrm{f}$ ' describing the difference between the

options = optimset('TolX',1 e-20,'TolFun',1 e-32, 'Display', 'off'); \% alters the optimization parameters for the undeformed volume $\left(V_{0}\right)$ and the deformed volume $(V)$, the unknown variable ' $r$ ' is represented by ' $x$ '

rs $(i)=$ fzero(f,rrs,options $)$; function 'fzero'

end $\%$ finds rs such that platelet volume is preserved during aspiration

$\mathrm{pp}=\left(\right.$ ppo. ${ }^{*}$ rs-ppi. $\left.{ }^{*} \mathrm{rp}\right) . /(\mathrm{rs}-\mathrm{rp}) ; \quad$ \%evaluates intracellular pressure

$\%$ options for Levenberg-Marquardt method of solution options=optimset('MaxFunEvals', le100,'tolFun',1 e-5,'TolX', 1e 8,'MaxIter',5e3, 'LevenbergMarquardt','on');

warning off all; $\quad$ \%disables all warnings

switch species

case 'human'

iniconstants $=\left(\begin{array}{ll}4.70 & 1.47 \mathrm{e}-4 \\ -1.80 \mathrm{e}-5 & -8.430 \mathrm{e}-5)\end{array}\right) \%$ initial guesses for material constants (human)

$\mathrm{n} 2=3$

$\mathrm{n} 3=5$

case 'mouse'

iniconstants $=(3.891 .81 \mathrm{e}-4$ 9.85e-6 -4.94e-4); \% initial guesses for material constants (mouse)

$\mathrm{n} 2=1$

n3 = 3;

end

(Constants, resnorm, residual,exitflag,output,l, $\mathrm{J})=\ldots$

lsqnonlin(@errLC,iniconstants,(),(),options); \% the optimizer calls the @errLC m-file, using initial constants and imposing altered options set to generate vector of 'Constants'

fprintf(')n');

fprintf('MODEL CONSTANTS $\backslash n$ '); \% prints 'MODEL CONSTANTS' to the screen

$\mathrm{CI}=$ nlparci(Constants,residual,'jacobian',J); \% determines $95 \%$ confidence intervals for constants

$\%$ prints optimized material constants $\left(c_{i}, i=1-3, T_{0}\right)$ to the screen fprintf('c1 $=\% 12.8 \mathrm{f}+/-\% 12.8 \mathrm{fn}$ ', Constants $\left.(1), 0.5^{*}(\mathrm{CI}(1,2)-\mathrm{CI}(1,1))\right)$;

fprintf('c2 $=\% 12.8 \mathrm{f}+/-\% 12.8 \mathrm{fnn}$ ', Constants(2),0.5* $(\mathrm{CI}(2,2)-\mathrm{Cl}(2,1)))$;

fprintf('c3 $=\% 12.8 \mathrm{f}+/-\% 12.8 \mathrm{fnn}$ ', Constants(3), $\left.0.5^{*}(\mathrm{Cl}(3,2)-\mathrm{CI}(3,1))\right)$;

fprintf('To $=\% 12.8 \mathrm{f}+/-\% 12.8 \mathrm{fn}$ ',Constants(4), $0.5 *(\mathrm{CI}(4,2)-\mathrm{CI}(4,1)))$;

\section{1-Material Constants Identification/errLC:}




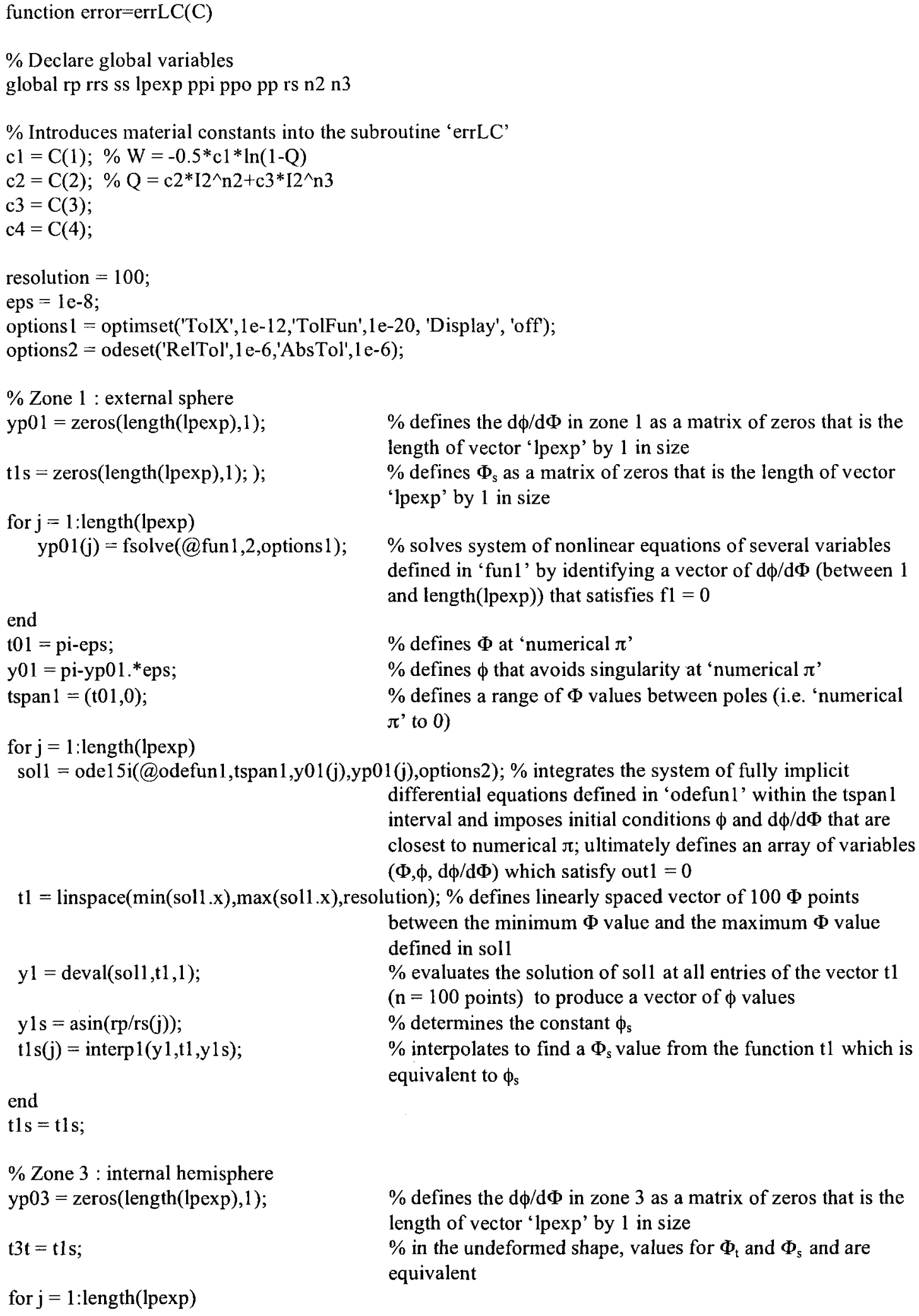

$\%$ defines the $d \phi / d \Phi$ in zone 3 as a matrix of zeros that is the length of vector 'lpexp' by 1 in size $\%$ in the undeformed shape, values for $\Phi_{\mathrm{t}}$ and $\Phi_{\mathrm{s}}$ and are equivalent 


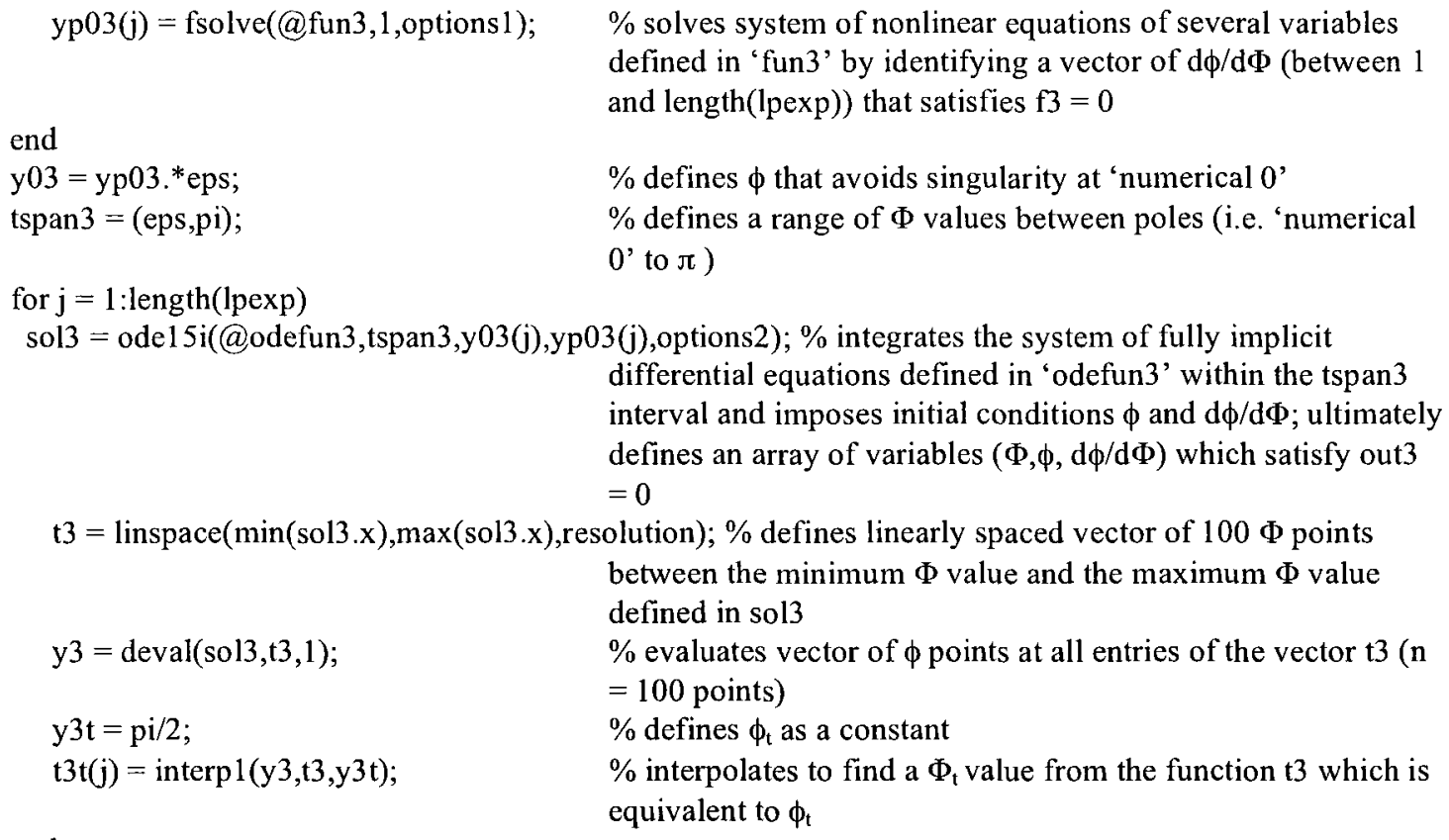

$\%$ Zone-dependent functions for 'fsolve' and 'odel $5 \mathrm{i}$ '

function $\mathrm{fl}=$ fun $1(\mathrm{x})$

$\mathrm{q}=\mathrm{c} 2 *\left((\mathrm{rs}(\mathrm{j}) / \mathrm{rrs})^{\wedge} 4^{*} \mathrm{x}^{\wedge} 4-1\right)^{\wedge} \mathrm{n} 2+\mathrm{c} 3 *\left((\mathrm{rs}(\mathrm{j}) / \mathrm{rrs})^{\wedge} 4^{*} \mathrm{x}^{\wedge} 4-1\right)^{\wedge} \mathrm{n} 3$ 


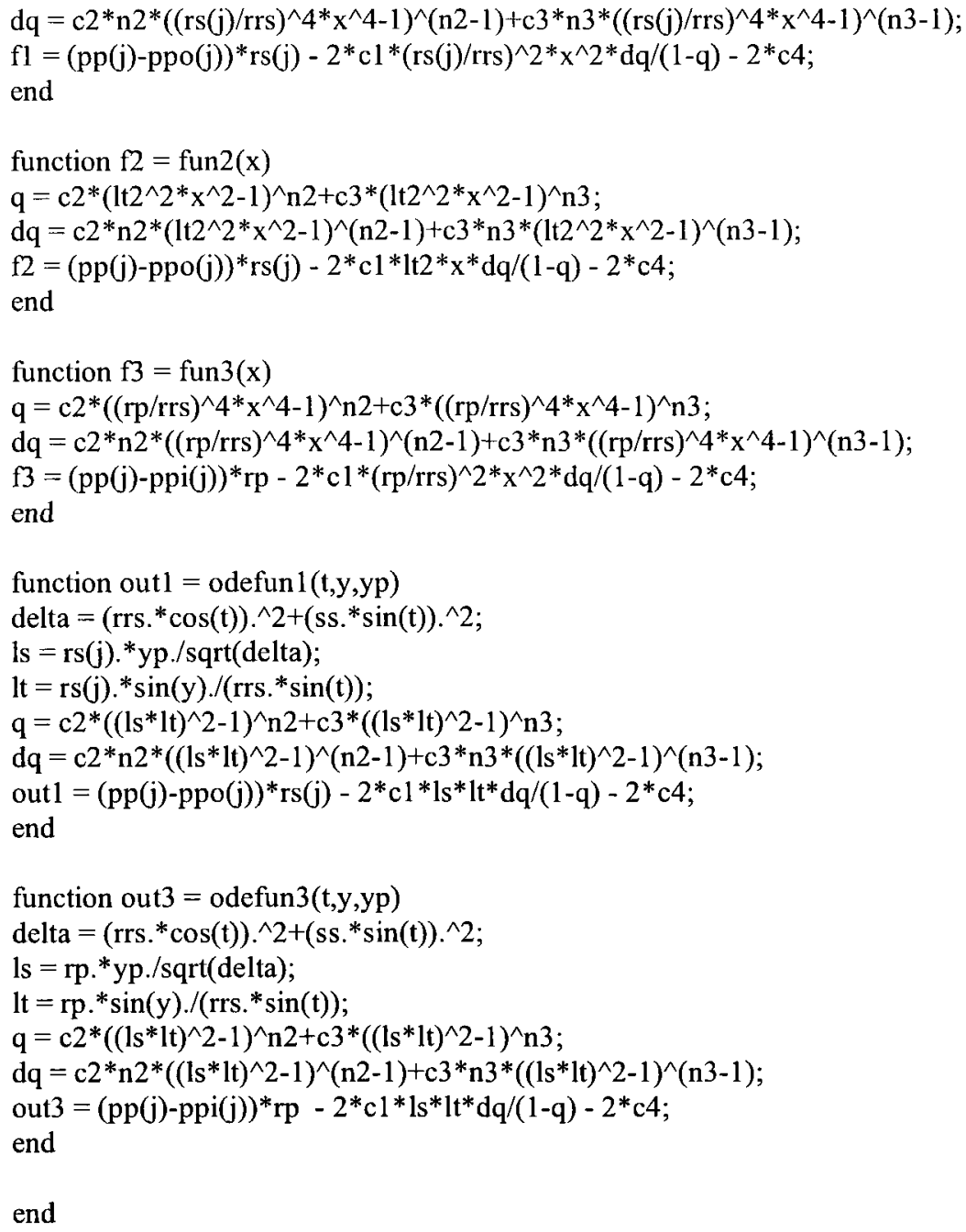

\section{2-Simulation for Pressure Range/main:}

clc

clear

format long

global rp rrs ss C ppi ppo v0 n2 n3

species = 'human';

$\%$ 'human' or 'mouse'

shaperatio $=0.15$; $\%$ shaperatio is ss/rrs, and is between 0.15 and 0.25 , based on
info from human platelets

$\%$ Read in raw experimental data from rawdata txt switch species case 'human' fid = fopen('rawdatahuman.txt', 'rt'); case 'mouse'

end

$$
\text { fid = fopen('rawdatamouse.txt', 'rt'); }
$$




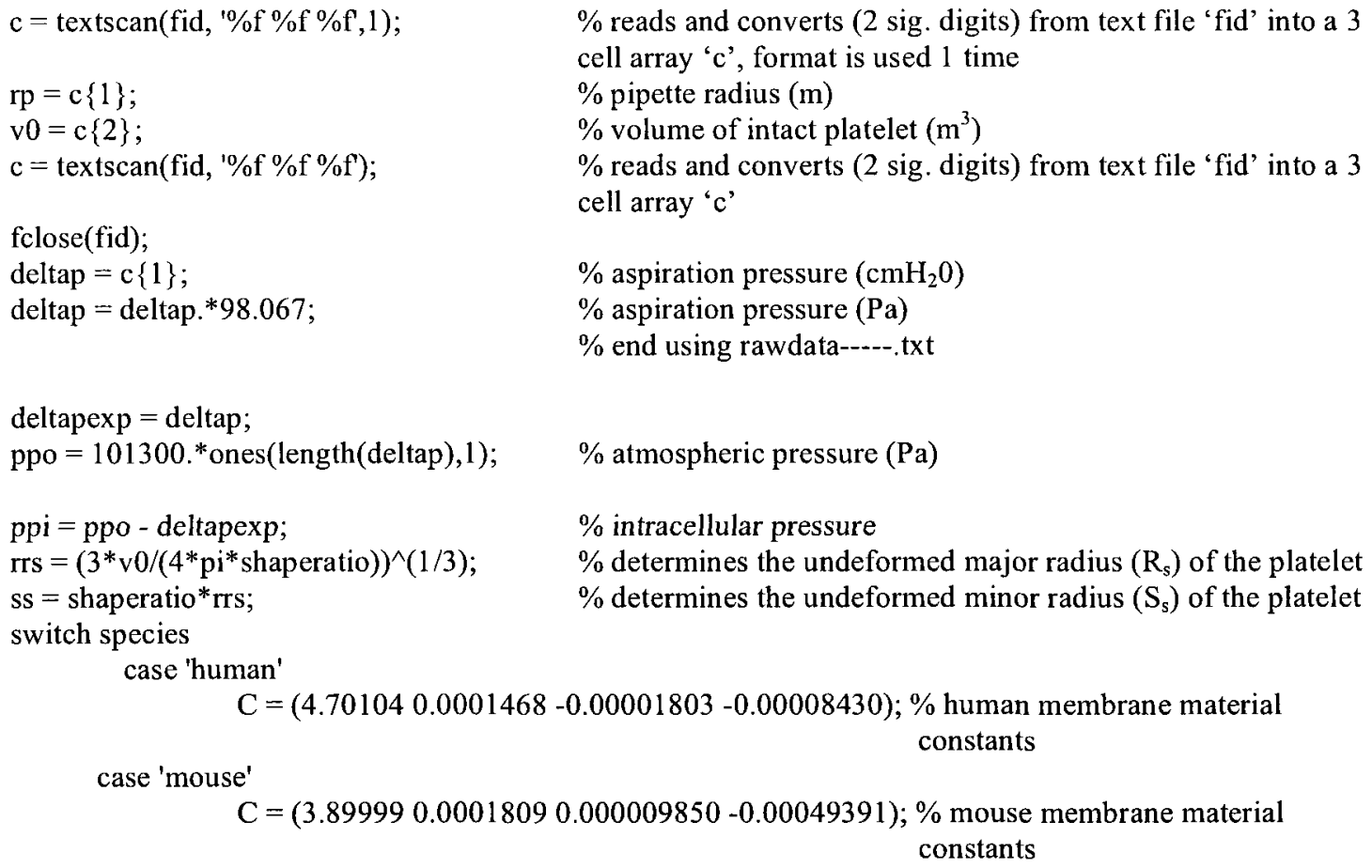

end

$\%$ options for Levenberg-Marquardt method of solution

options=optimset('MaxFunEvals',1 e100,'tolFun',1 e-2,'TolX', 1 e-2,'MaxIter', 5 e3,'LevenbergMarquardt','on');

warning off all;

$\%$ Using optimized material constants, known geometry, and pressure range, generate optimized platelet tongue lengths $\left(\mathrm{L}_{\mathrm{p}}\right)$ compatible with a conservation of platelet volume

$\mathrm{lb}=1.1{ }^{*}$ ones(length(deltap),1). ${ }^{*} \mathrm{rp} ; \quad \%$ defines a matrix with values that exceed the micropipette radius ( $=1.1 \mathrm{rp}$ ) that is the length of vector 'deltap' by 1 in

switch species size

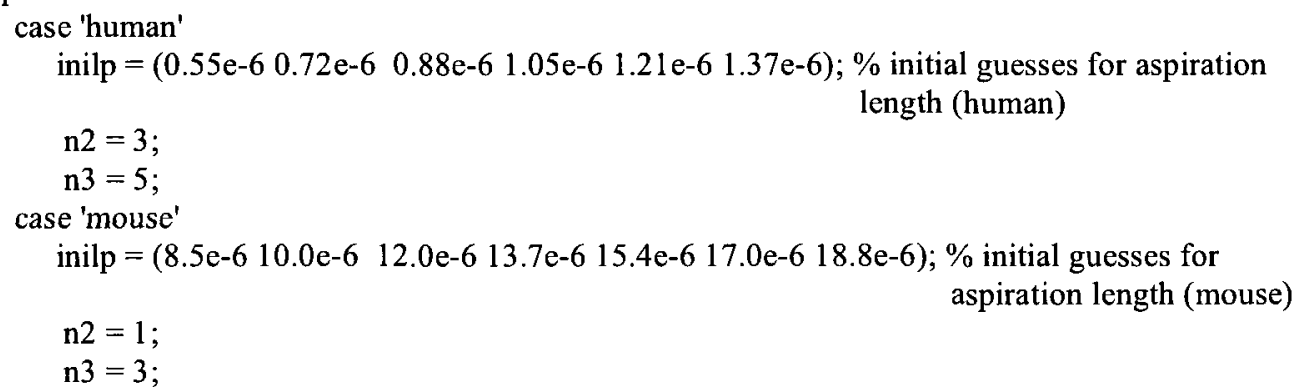


for $\mathrm{i}=1:$ length $(\mathrm{lp})$

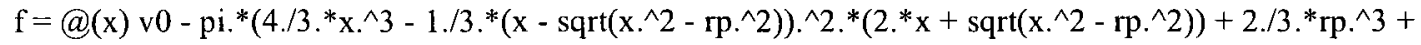

(lp(i) - rp).*rp.^2); \% defines a function ' $\mathrm{f}$ ' describing the difference between the undeformed volume $\left(\mathrm{V}_{\mathrm{o}}\right)$

and the deformed volume $(\mathrm{V})$, the unknown variable ' $r s$ ' is represented by ' $\mathrm{x}$ '

options = optimset('TolX',1 e-20,'TolFun',1 e-32, 'Display', 'off'); \% alters the optimization parameters for the function 'fzero'

rs $(i)=$ fzero(f,rrs,options); $\quad$ \% finds rs such that platelet volume is preserved during

end aspiration

\section{2-Simulation for Pressure Range/errLlp:}

function error=errLlp $(l p)$

$\%$ Declare global variables

global rp rrs ss C ppi ppo v0 n2 n3

$\%$ The following development is identical to that detailed for the $\mathrm{m}$-file ' 1 -Material Constants Identification/errLC', save the evaluation of a theoretical aspiration length (lp) rather than the experimentally derived alternative (lpexp).

$\mathrm{cl}=\mathrm{C}(1) ; \% \mathrm{~W}=-0.5 * \mathrm{cl} * \ln (1-\mathrm{Q})$

$\mathrm{c} 2=\mathrm{C}(2) ; \% \mathrm{Q}=\mathrm{c} 2 * \mathrm{I} 22^{\wedge} \mathrm{n} 2+\mathrm{c} 3 * \mathrm{I} 2^{\wedge} \mathrm{n} 3$

$\mathrm{c} 3=\mathrm{C}(3)$;

$\mathrm{c} 4=\mathrm{C}(4)$;

rs=zeros(length(lp),1);

for $\mathrm{i}=1$ :length $(\mathrm{lp})$

$\mathrm{f}=@(\mathrm{x}) \mathrm{v} 0-\mathrm{pi} \mathbf{i}^{*}\left(4 / 3^{*} \mathrm{x}^{\wedge} 3-1 / 3^{*}\left(\mathrm{x}-\operatorname{sgrt}\left(\mathrm{x}^{\wedge} 2-\mathrm{rp}^{\wedge} 2\right)\right)^{\wedge} 2^{*}\left(2^{*} \mathrm{x}+\operatorname{sqrt}\left(\mathrm{x}^{\wedge} 2-\mathrm{rp}^{\wedge} 2\right)\right)+2 / 3^{*} \mathrm{rp}^{\wedge} 3+(\operatorname{lp}(\mathrm{i})-\right.$ $\left.\mathrm{rp})^{*} \mathrm{rp}^{\wedge} 2\right)$

options = optimset('TolX',1e-20,'TolFun',1e-32, 'Display', 'off');

rs(i) $=$ fzero $(f, r r s, o p t i o n s)$;

end

aspiration

$\mathrm{pp}=\left(\right.$ ppo. $\left.{ }^{*} \mathrm{rs}-\mathrm{ppi} .{ }^{*} \mathrm{rp}\right) . /(\mathrm{rs}-\mathrm{rp})$

resolution $=100$

eps = $1 \mathrm{e}-8$;

options 1 = optimset('TolX',1e-12,'TolFun',1e-20, 'Display', 'off');

options2 = odeset('RelTol',1 e-6,'AbsTol',1 e-6);

$\%$ Zone 1 : external sphere

yp01 = zeros(length(lp),1);

tls = zeros(length(lp),1);

for $\mathrm{j}=1$ : length(lp)

yp01(j)=fsolve(@fun1,2,options1);

end

t01 = pi-eps;

y01 = pi-yp01.*eps;

tspan1 $=(\mathrm{t} 01,0)$

for $\mathrm{j}=1$ :length $(\mathrm{lp})$

sol1=ode15i(@,odefun 1,tspan 1,y01(j),yp01(j),options2);

$\mathrm{t} 1$ = linspace $(\min (\operatorname{soll} . \mathrm{x}), \max ($ soll. $\mathrm{x})$, resolution);

$\mathrm{y} 1=\operatorname{deval}(\operatorname{sol} 1, \mathrm{t} 1,1)$; 


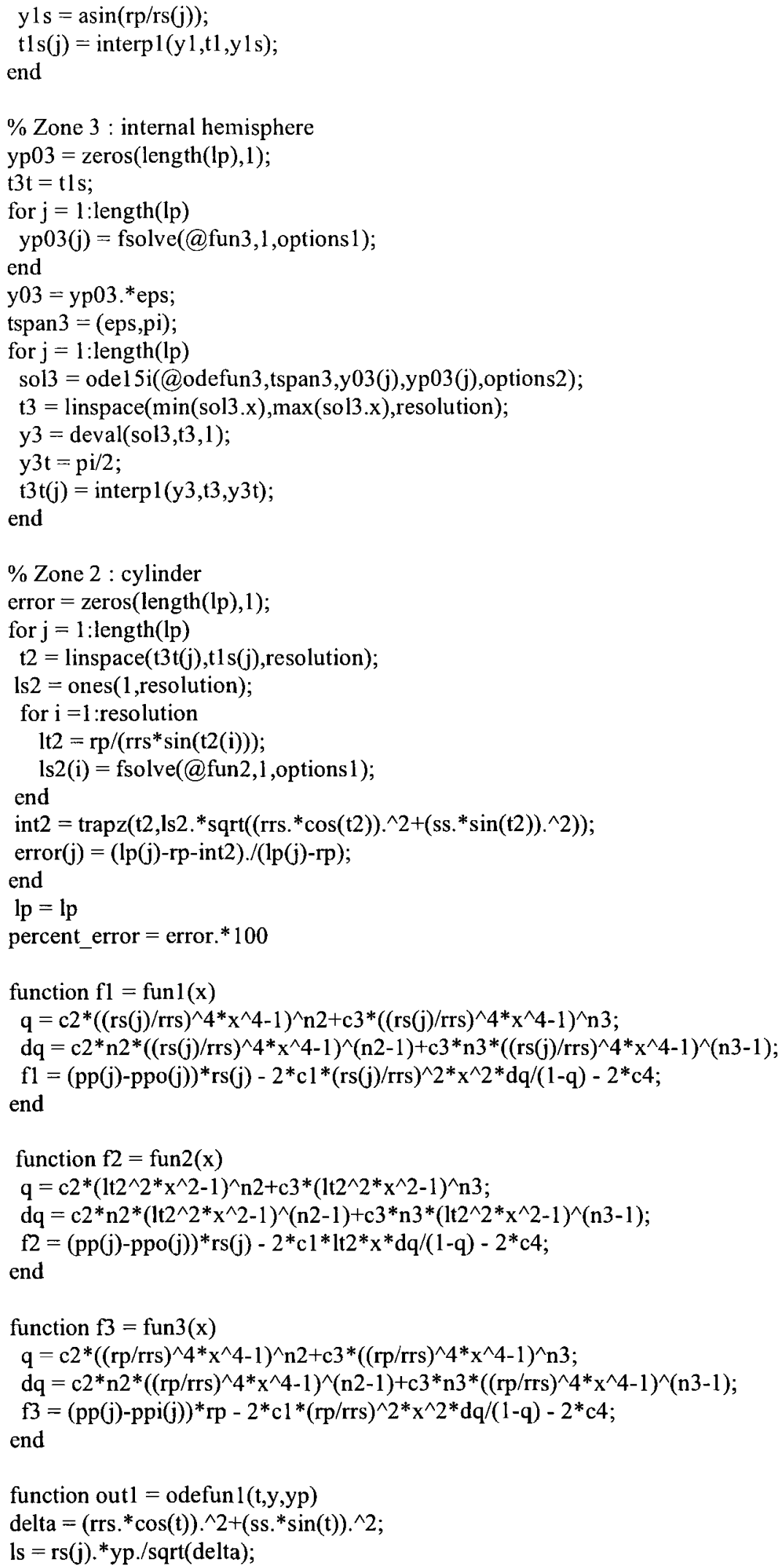




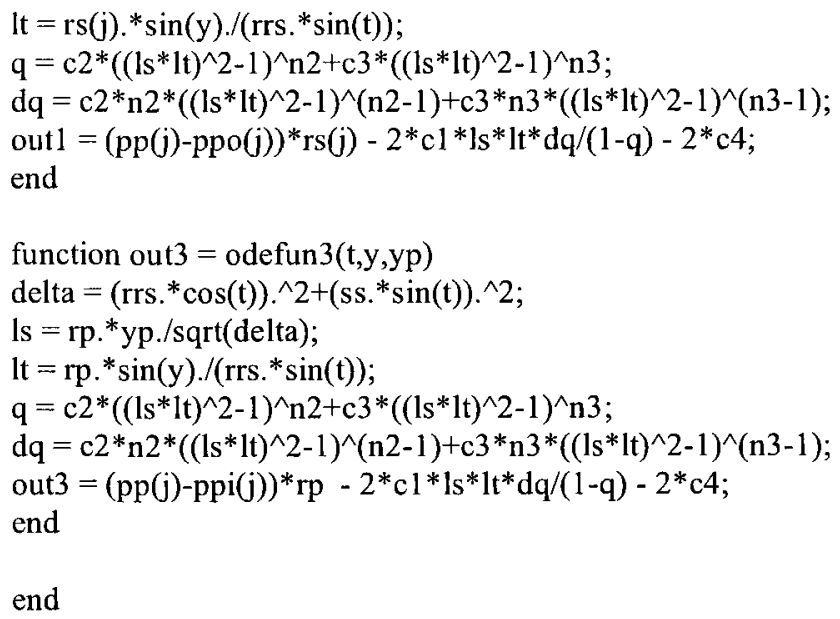


end

rrs $=\left(3 * v 0 /\left(4^{*} \mathrm{pi}^{*} \text { shaperatio }\right)\right)^{\wedge}(1 / 3)$

ss $=$ shaperatio*rrs;

switch species

eps $=1 \mathrm{e}-8$;

resolution $=1000$

$\mathrm{e}=\operatorname{sqrt}\left(\mathrm{rrs} \wedge^{\wedge} 2\right.$-ss.^2)./rrs;

s0 $=2 .{ }^{*}$ pi. ${ }^{*}$ rrs. ${ }^{\wedge} 2+$ pi. ${ }^{*}$ ss. ${ }^{\wedge} 2 .{ }^{*} \log ((1+\mathrm{e}) . /(1-\mathrm{e})) . / \mathrm{e} ; \%$ calculates undeformed platelet surface area

fid $=1$

fprintf(fid,'v0 = \% 10.8e m^3; s0=\%10.8e m^2 $\left.\backslash \mathrm{n}^{\prime}, \mathrm{v} 0, \mathrm{~s} 0\right)$;

switch species

case 'human'

$\mathrm{lp}=(10406 *($ deltap*rp $)+0.9703) * \mathrm{rp} ; \%$ for human platelets (from Haga et al. (11)) case 'mouse'

$1 \mathrm{p}=\left(146100^{*}\left(\text { deltap }^{*} \mathrm{rp}\right)+19.923\right)^{*} \mathrm{rp} ; \%$ for mouse platelets (from 11 of our samples)

end

fprintf(fid,'Lp $\left.=\% 10.8 \mathrm{e} \mathrm{m}^{\prime} \mathbf{n}^{\prime}, \mathrm{lp}\right)$;

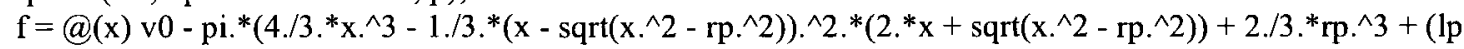
-rp).*rp.^2);

$\%$ defines a function ' $\mathrm{f}$ ' describing the difference between the undeformed volume $\left(V_{0}\right)$ and the deformed volume $(V)$, the unknown variable ' $r$ ' is represented by ' $x$ '

options = optimset('TolX',le-20,'TolFun',l e-32, 'Display', 'off); \% alters the optimization parameters for

rs = fzero(f,rrs,options); $\quad \%$ finds rs such that platelet volume is preserved during aspiration

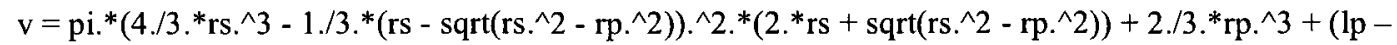

rp).*rp.^2); $\%$ calculates the deformed platelet volume as a verification that $\mathrm{V}_{\mathrm{o}}=\mathrm{V}$

$\mathrm{s}=$ pi. ${ }^{*}\left(2 .{ }^{*} \mathrm{rs} .{ }^{\wedge} 2+2 .{ }^{*} \mathrm{rs} .{ }^{*} \mathrm{sqrt}\left(\mathrm{rs} .{ }^{\wedge} 2-\mathrm{rp} .{ }^{\wedge} 2\right)+2 .{ }^{*} \mathrm{rp} .{ }^{\wedge} 2+2 .{ }^{*}(\mathrm{lp}-\mathrm{rp}) .{ }^{*} \mathrm{rp}\right) ; \%$ calculate the deformed platelet surface area, proof of change in SA following deformation

fprintf(fid, 'The resulting rs and areal change are: $\left.\mid n^{\prime}\right)$;

fprintf(fid,'rs $=\% 10.8 \mathrm{e} \mathrm{m} ;$ p.c. $\left.(\mathrm{dA} / \mathrm{dA0} 0-1)=\% 10.8 \mathrm{e} / \mathrm{n}^{\prime}, \mathrm{rs}, 100 .{ }^{*}(\mathrm{~s}-\mathrm{s} 0) / \mathrm{s} 0\right)$;

fprintf(fid,'v $\left.=\% 10.8 \mathrm{e} \mathrm{m} \mathrm{m}^{\wedge} ; \mathrm{s}=\% 10.8 \mathrm{e} \mathrm{m}^{\wedge} 2 \backslash \mathrm{n}^{\prime}, \mathrm{v}, \mathrm{s}\right)$;

$\mathrm{pp}=\left(\right.$ ppo. ${ }^{*}$ rs-ppi. $\left.{ }^{*} \mathrm{rp}\right) . /(\mathrm{rs}-\mathrm{rp})$

options1 = optimset('TolX',1 e-12,'TolFun',1e-20, 'Display', 'off');

options2 = odeset('RelTol',1 e-6,'Abs Tol',1 e-6);

warning off all;

$\%$ Zone 1 : external sphere

yp01 = fsolve(@fun1,2,options 1$) ; \quad$ \% solves system of nonlinear equations of several variables

t01 = pi-eps;

y01 = pi-yp01.*eps; defined in 'fun 1 ' by identifying a scalar value of $d \phi / d \Phi$

$\%$ defines $\Phi$ at 'numerical $\pi$ '

$\%$ defines $\phi$ that avoids singularity at 'numerical $\pi$ ' 


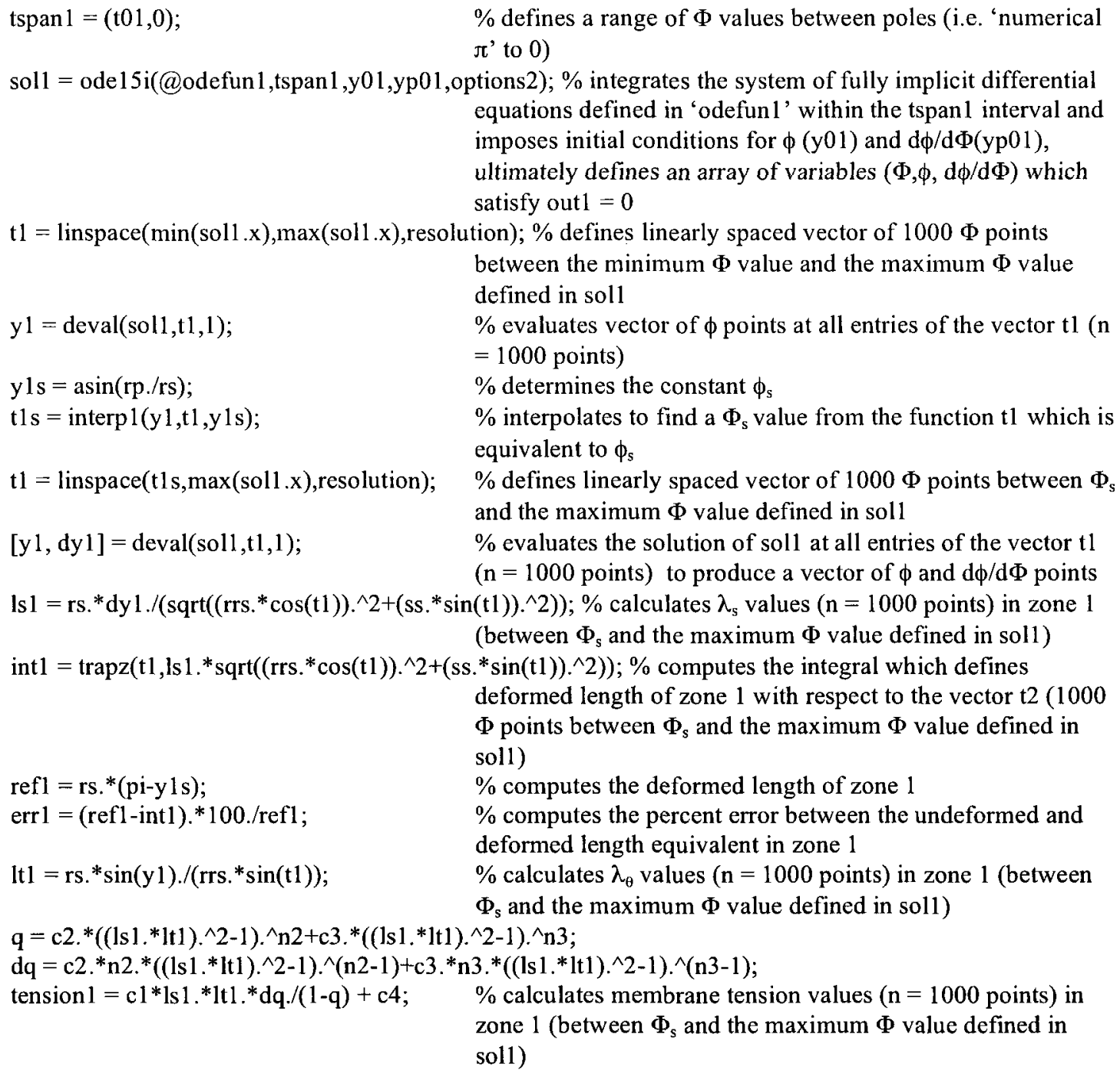




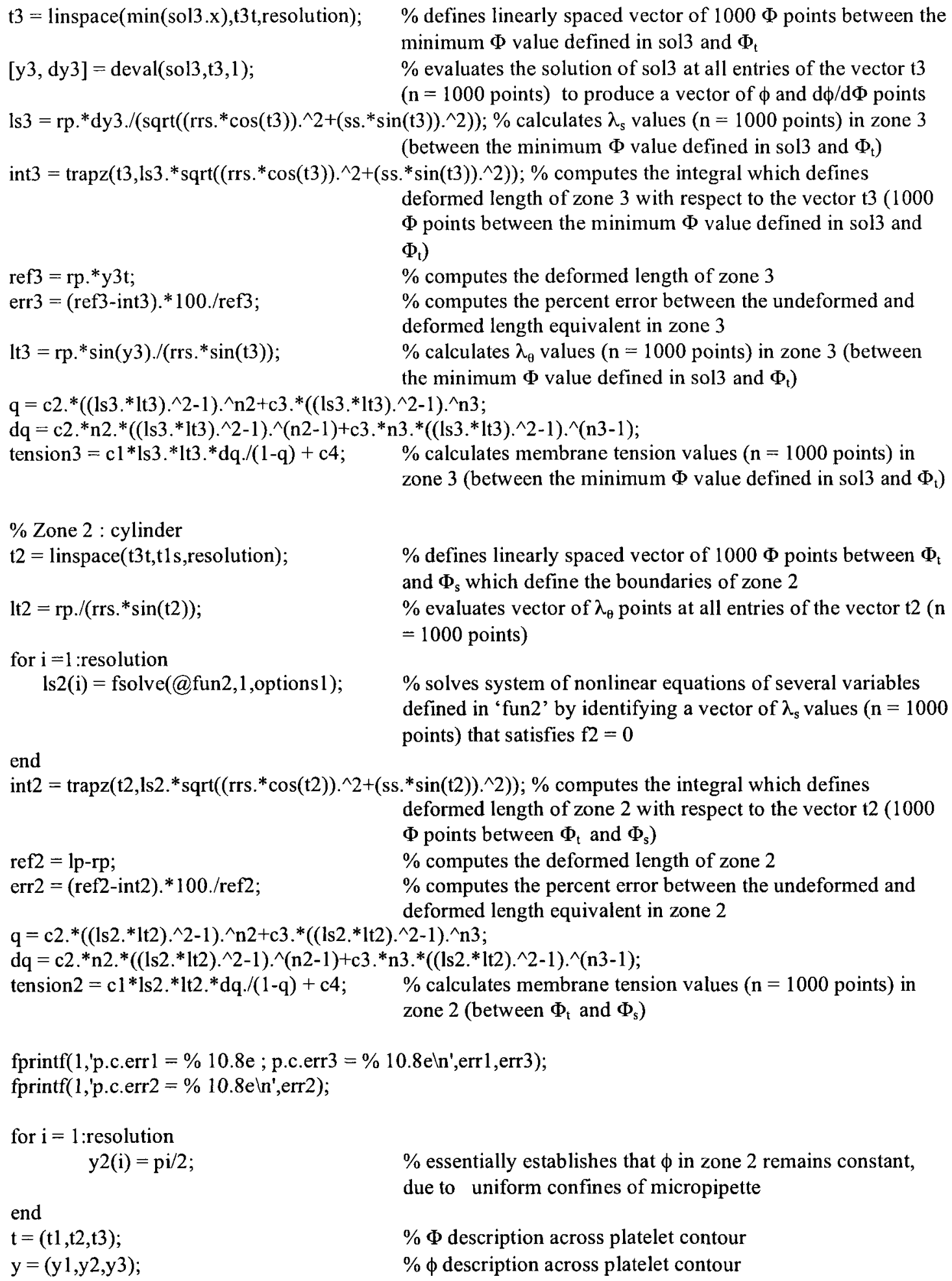

$\%$ Zone-dependent values for radial and axial lengths for the deformed platelet $\mathrm{rr} 1=\mathrm{rs} .{ }^{*} \sin (\mathrm{y} 1)$; 


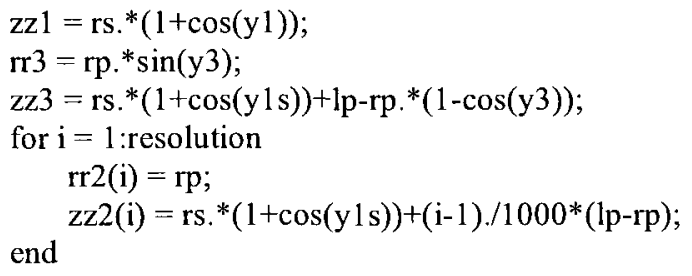

figure

plot((t1 t 2 t3), (tension 1 tension2 tension 3$\left.),{ }^{\prime}.\right)$;

xlabel('Angle between normal to surface and vertical (rad)')

ylabel('Membrane tension $\left.(\mathrm{N} / \mathrm{m})^{\prime}\right)$

figure

plot((t1 t2 t3),(1s1 ls2 ls3),'b.',(t1 t2 t3),(1t1 1t2 lt3),'g*');

xlabel('Angle between normal to surface and vertical (rad)')

ylabel('Longitudinal and circumferential stretches $(-)$ ')

figure

plot((t1 t2 t3),(ls1.*1t1 ls2.*1t2 ls3.*lt3), .');

xlabel('Angle between normal to surface and vertical (rad)')

ylabel('Product of longitudinal and circumferential stretches (-)')

figure

plot(rr0,zz0,'',rr0s,zz0s,'y+',rr0t,zz0t,'r+',r,z,',',current_rr0s,current_zz0s,'y+',current_rr0t,current_zz0t,'r+')

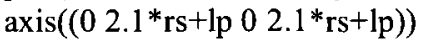

$\%$ title('Undeformed (blue) and deformed (green) platelet shapes')

xlabel('r (m)')

ylabel('z (m)')

\section{3-Various Results at Single Pressure/fun1:}

function $\mathrm{fl}=$ fun $1(\mathrm{x})$ 
global c1 c2 c3 c4 n2 n3 rs rrs pp ppo

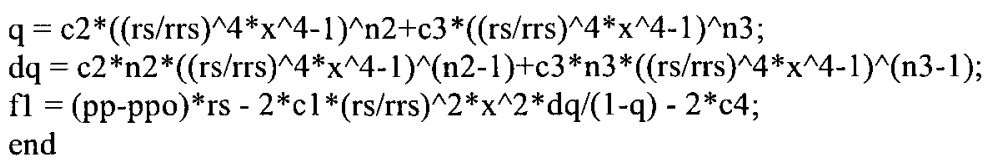

\section{3-Various Results at Single Pressure/fun2:}

function $\mathrm{f} 2=$ fun $2(\mathrm{x})$

global cl c2 c3 c4 n2 n3 rs lt2 pp ppo i

$\mathrm{q}=\mathrm{c} 2 *\left(1 \mathrm{t} 2(\mathrm{i})^{\wedge} 2^{*} \mathrm{x}^{\wedge} 2-1\right)^{\wedge} \mathrm{n} 2+\mathrm{c} 3 *\left(1 \mathrm{t} 2(\mathrm{i})^{\wedge} 2 * \mathrm{x}^{\wedge} 2-1\right)^{\wedge} \mathrm{n} 3$

$\mathrm{dq}=\mathrm{c} 2 * \mathrm{n} 2 *\left(\operatorname{lt} 2(\mathrm{i})^{\wedge} 2^{*} \mathrm{x}^{\wedge} 2-1\right)^{\wedge}(\mathrm{n} 2-1)+\mathrm{c} 3{ }^{*} \mathrm{n} 33^{*}\left(\mathrm{lt} 2(\mathrm{i})^{\wedge} 2^{*} \mathrm{x}^{\wedge} 2-1\right)^{\wedge}(\mathrm{n} 3-1)$;

$\mathrm{f} 2=(\mathrm{pp}-\mathrm{ppo})^{*} \mathrm{rs}-2^{*} \mathrm{c} 1^{*} \operatorname{lt} 2(\mathrm{i})^{*} \mathrm{x}^{*} \mathrm{dq} /(1-\mathrm{q})-2^{*} \mathrm{c} 4$;

end 


\section{Bibliography}

(1) Kroll M, Hellums JD, McIntire LV, Schafer AI and Moake JL. 1996. Platelets and shear stress. Blood. 88:1525-41.

(2) Goldhaber SZ. 2000. Diagnosis of deep vein thrombosis. Clinical Cornerstone. 2:29-37.

(3) Kassaï B, Boissel J-P, Cuchera M, Sonie S. Shah NR, and Leizorovicz A. 2004. A systematic review of the accuracy of ultrasound in the diagnosis of deep vein thrombosis in asymptomatic patients. Thrombosis \& Haemostasis. 91:655-666.

(4) Becker RC. 2002. Anticoagulation and the heart. Journal of Thrombosis and Thrombolysis. 12:41-52.

(5) Anand M, Rajagopal K, and Rajagopal KR. 2004. A model incorporating some of the mechanical and biochemical factors underlying clot formation and dissolution in flowing blood. Journal of Theoretical Medicine. 5:183-218.

(6) Bluestein D, Gutierrez C, and Londono $\mathrm{M}$ et al. 1999. Vortex shedding in arterial stenosis and its relevance to mural platelet deposition. Annals of Biomedical Engineering. 27:763-73.

(7) Bluestein D, Yin W, Affeld K, and Jesty J. 2004. Flow induced platelet activation in mechanical heart valves. The Journal of Heart Valve Disease. 13:501-8.

(8) Rothrock JF, and Hart RG. 1991. Antithrombotic therapy in cerebrovascular disease. Annals of Internal Medicine. 115:885-895.

(9) Leibson CL, Petterson TM, Bailey KR, Melton J, and Heit JA. 2008. Risk factors for venous thromboembolism in nursing home residents. Mayo Clin Proceedings. 83:151-157.

(10) Gimbrone M. 1999. Endothelial dysfunction, hemodynamic forces and atherosclersosis. Thrombosis \& Haemostasis. 82:722-26.

(11) Haga JH, Beaudoin AJ, White JG, and Strony J. 1998. Quantification of the passive mechanical properties of the resting platelet. Annals of Biomedical Engineering. 26:268-277. 
(12) Zhang J, Bergeron A, Yu Q, Sun C, McBride L, Bray P, and Dong J. 2003. Duration of exposure to high fluid shear stress is critical in shear-induced platelet activation-aggregation. Thrombosis \& Haemostasis. 90:672-8.

(13) Marieb E. 2004. Human Anatomy \& Physiology. San Francisco: Pearson Benjamin Cummings.

(14) Michelson A. 2007. Platelets. Burlington: Elsevier Inc.

(15) Boyles J, Fox JEB, Phillips DR, and Stenberg PE. 1985. Organization of the cytoskeleton in resting, discoid platelets: Preservation of actin filaments by a modified fixation that prevents osmium damage.. The Journal of Cell Biology. 101:1463-1472.

(16) Kamath S, Blann AD, and Lip YH. 2001. Platelet activation: assessment and quantification. European Heart Journal. 22:1561-1571.

(17) Rosing J, Van Rijn JL, and Beevers EM et al. 1985. The role of activated human platelets in prothrombin and factor X activation. Blood. 65:319-332.

(18) Escolar G, and White JG. 1991. The platelet open canalicular system: A final common pathway. Blood. 17:467-485.

(19) Fukami MH, and Salganicoff L. 1977. Human platelet storage organelles. A review. Thrombosis \& Haemostasis. 38: 963-970.

(20) Konstantopoulos K, Neelamegham S, Burns AR, Hentzen E, Kansas GS, Snapp KR, Berg EL, Hellums JD, Smith CW, McIntire LV, and Simon SI. 1998. Venous levels of shear support neutrophil-platelet adhesion and neutrophil aggregation in blood via p-selectin and beta2-integrin. Circulation. 98:873-82.

(21) Loscalzo, J. 2002. Oxidative stress in endothelial cell dysfunction and thrombosis. Pathophysiology of Haemostasis and Thrombosis. 32:359-360.

(22) Huang JN, and Koerper MA. 2008. Factor V deficiency: a concise review. Haemophilia. 14:1164-9.

(23) Bickfalvi, A. 2004. Platelet factor 4: An inhibitor of angiogenesis. Seminars in Thrombosis and Hemostasis. 30:379-85.

(24) Brandt E, Petersen F, Ludwig A, Ehlert JE, Bock L, and Flad HD. 2000. The $\beta$ thromboglobulins and platelet factor 4: blood-derived CXC chemokines with divergent roles in early neutrophil regulation. Journal of Leukocyte Biology. 67:471-78. 
(25) Van der Linden, P, and Ickx, BE. 2006. The effects of colloid solutions on hemostasis. Canadian Journal of Anaesthesia. 53:S30-9.

(26) de Jonge E, and Levi M. 2001. Effects of different plasma substitutes on blood coagulation: A comparative review. Critical Care Medicine. 29:1261-7.

(27) Ruggeri ZM, de Marco L, Gatti L, Bader R, and Montgomery RR. 1983. Platelets have more than one binding site for von Willebrand Factor. Journal of Clinical Investigation. 72:1-12.

(28) McEver RP. 1990a. The clinical significance of platelet membrane glycoproteins. Hematology/Oncology Clinics of North America. 4:87-105.

(29) Stassen J, Arnout J, and Deckmyn H. 2004. The hemostatic system. Current Medicinal Chemistry. 11:2245-60.

(30) Furman M, Barnard MR, Krueger LA, Fox ML, Shilale EA, Lessard DM, Marchese P, Frelinger III AL, Goldberg RJ, and Michelson AD. 2001. Circulating monocyte-platelet aggregates are an early marker of acute myocardial infarction. Journal of the American College of Cardiology. 38:1002-6.

(31) McEver R. 1990b. Properties of GMP-140, an inducible granule membrane protein of platelets and endothelium. Blood Cells. 16:73-80.

(32) Slack S, and Turitto VT. 1993. Fluid dynamic and hemorheologic considerations. Cardiovascular Pathology Supplements.. 2:11-21.

(33) Feng S, Christodoulides N, Resendiz JC, Berndt MC, and Kroll MH. 2000. Cytoplasmic domains of Gplbalpha and Gplbbeta regulate 14-3-3zeta binding to $\mathrm{GpIb} / \mathrm{IX} / \mathrm{V}$. Blood. 95:551-7.

(34) Sakariassen K, Nievelstein P, Coller B, and Sixma J. 1986. The role of platelet membrane glycoproteins $\mathrm{Ib}$ and IIb-IIIa in platelet adherence to human artery subendothelium. British Journal of Haematology. 63:681-91.

(35) Shattil S. 1999. Signaling through platelet integrin allbb3: inside out, outside in and sideways. Thrombosis \& Haemostasis. 82:318-25.

(36) McManama G, Lindon $\mathrm{N}$, and Kloczewiak $M$ et al. 1986. Platelet aggregation by fibrinogen polymers cross-linked across the E domain. Blood. 68:363-71.

(37) Travis B, Marzec UM, and Ellis JT et al. 2001. The sensitivity of indicators of thrombosis initiation to a bileaflet prosthesis leakage stimulus. The Journal of Heart Valve Disease. 10:228-38. 
(38) Schmitt A, Guichard J, Massé J-M, Debili N, and Cramer EM. 2001. Of mice and men: Comparison of the ultrastructure of megakaryocytes and platelets. Experimental Hematology. 29:1295-302.

(39) Subramaniam M, Frenette PS, Saffaripour S, Johnson RC, Hynes RO, and Wagner DD. 1996. Defects in hemostasis in P-selectin deficient mice. Blood. 87:123842 .

(40) Kato K, Martinez C, Russell S, Nurden P, Nurden A, Fiering S, and Ware J. 2004. Genetic deletion of mouse platelet glycoprotein Ibbeta produces a BernardSoulier phenotype with increased alpha-granule size. Blood. 104:2339-44.

(41) Lewis, JH. 1996. Rodents. In: Comparative Hemostasis in Vertebrates. New York: Lewis JH, eds.

(42) Tsakiris DA, Scudder L, Hodivala-Dilke K, Hynes RO, and Coller BS. 1999. Hemostasis in the mouse (Mus musculus): A review. Thrombosis \& Haemostasis: $81: 177-88$.

(43) Novak EK, Hui SW and Swank RT. 1984. Platelet storage pool deficiency in mouse pigment mutations associated with seven distinct genetic loci. Blood. 63:536-44.

(44) Smyth SS, Tsakiris DA, Scudder LE, and Coller BS. 2000. Structure and function of murine alphaIIbeta3 (GPIIb/IIIa): studies using monoclonal antibodies and beta-3-null mice. Thrombosis \& Haemostasis. 84:1103-8.

(45) Sambrano GR, Weiss EJ, Zheng YW, Huang W, and Coughlin SR. 2001. Role of thrombin signaling in platelets in haemostasis and thrombosis. Nature. 413:74-8.

(46) Jirouskova M, Shet AS, and Johnson GJ. 2007. A guide to murine platelet structure, function, assays, and genetic alterations. Journal of Thrombosis and Haemostasis. 5:661-9.

(47) Emeis JJ, Jirouskova M, Muchitsch E-M, Shet AS, Smyths SS, and Johnson GJ. 2007. A guide to murine coagulation factor structure, function, assays, and genetic alterations. Journal of Thrombosis and Haemostasis. 5:670-9.

(48) Roth G, and Calverley DC. 1994. Aspirin, platelets, and thrombosis: Theory and practice. Blood. 83:885-898.

(49) Ramstack J, Zuckerman, L, and Mockros L. 1979. Shear induced activation of platelets. Journal of Biomechanics. 12:113-125. 
(50) Jesty J, Win Y, Perrotta P, and Bluestein D. 2003. Platelet activation in a circulating flow loop: combined effects of shear stress and exposure time. Platelets. 14:1439.

(51) Sutera S, Nowak MD, and Joist JH et al.. 1988. A programmable, computercontrolled, cone-plate viscometer for the application of pulsatile shear stress to platelet suspensions. Biorheology. 25:449-59.

(52) Boreda R, Tatemi RS, and Rittgers SE. 1995. Potential for platelet stimulation in critically stenosed carotid and coronary arteries. Journal of Vascular Investigation. 1:26-37.

(53) Turitto V. 1982. Blood viscosity, mass transport, and thrombogenesis. Progress in Hemostasis and Thrombosis. 6:139-77.

(54) Bluestein D, Niu L, and Schoephoerster RT et al.. 1997. Fluid mechanics of flow through a stenosis: relationship to the development of mural thrombus. Annals of Biomedical Engineering. 25:344-356.

(55) Wootton D, Markou C, Hanson S, and Ku D. 2001. A mechanistic model of acute platelet accumulation in thrombogenic stenoses. Annals of Biomedical Engineering. 29:321-329.

(56) Zhang J, Bergeron A, Yu Q, Sun C, McIntire L, Lopez J, and Dong J. 2002. Platelet aggregation and activation under complex patterns of shear stress. Thrombosis \& Haemostasis. 88:817-21.

(57) Chow T, Hellums JD, Moake JL, and Kroll MH. 1992. Shear stress-induced von Willebrand factor binding to platelet glycoprotein $\mathrm{Ib}$ initiates calcium influx associated with aggregation. Blood. 80:113-20.

(58) Wurzinger L, Opitz R, Wolf M, and Schmid-Schönbein, H. 1987. Ultrastructural Investigations on the Question of Mechanical Activation of Blood Platelets. Blut. 54:97-107.

(59) Doggett T, Girdhar G, Lawshé A, Schmidtke DW, Laurenzi IJ, Diamond SL, and Diacovo TG. 2002. Selectin-like kinetics and biomechanics promote rapid platelet adhesion in flow: The GpIba-vWF tether bond. Biophysical Journal. 83:194-205.

(60) Edmunds LJ. 1987. Thrombotic and bleeding complications of prosthetic heart valves. The Annals of Thoracic Surgery. 44:430-45.

(61) Johansen P. 2004. Mechanical heart valve cavitation. Expert Reviews of Medical Devices. 1:95-104. 
(62) Pumphrey C, and Dawes J. 1983. Platelet alpha granule depletion: Findings in patients with prosthetic heart valves and following cardiopulmonary bypass surgery. Thrombosis Research. 30:257-64.

(63) Bluestein D, Li Y, and Krukenkamp JB. 2002. The role of wake dynamics in enhancing the risk of cardioembolism in mechanical heart valves-a transient and turbulent analysis. Journal of Biomechanics. 35:1533-40.

(64) Peterson D, Stathopoulos NA, Giorgio TD, Hellums JD, and Moake JL. 1987. Shear-induced platelet aggregation requires von Willebrand factor and platelet membrane glycoproteins Ib and IIb-IIIa. Blood. 69:625-8.

(65) White J, Burris SM, Hasegawa D, and Johnson M. 1984a. Micropipette aspiration of human blood platelets: A defect in Bernard-Soulier syndrome. Blood. 63:1249-52.

(66) White J, Burris SM, Tukey D, Smith II C, and Clawson CC. 1984b. Micropipette aspiration of human platelets: Influence of microtubules and actin filaments on deformability. Blood. 63:210-4.

(67) Fox J, Baughan AK, and Phillips DR. 1983. Direct linkage of GPIb to a $\mathrm{M}_{\mathrm{r}}=$ 250,000 polypeptide in platelet cytoskeletons. Blood. 62 (Suppl):255a.

(68) Ethier C, Simmons CA. 2007. Introductory biomechanics: From cells to organisms: Cambridge: Cambridge University Press.

(69) Maniotis A, Chen CS, and Ingber DE. 1997. Demonstration of mechanical connections between integrins, cytoskeletal filaments, and nucleoplasm that stabilize nuclear structure. Proceedings of the National Academy of Sciences of the USA. 94:849-54.

(70) Nachmias V. 1980. Cytoskeleton of human platelets at rest and after spreading. Journal of Cell Biology. 86:795-802.

(71) Bennett J, Zigoind S, Vilaire G, Cunningham ME, and Bednar B. 1999. The platelet cytoskeleton regulates the affinity of the integrin allbb3 for fibrinogen. The Journal Biological Chemistry. 274:25301-7.

(72) Ramasamy I. 2004. Inherited bleeding disorders: disorders of platelet adhesion and aggregation. Critical Reviews of Oncology/Hematology. 49:1-35.

(73) Savage B, Almus-Jacobs F, and Ruggeri ZM. 1998. Specific synergy of multiple substrate-receptor interactions in platelet thrombus formation under flow. Cell. 94:657-66.

(74) Arya M, Anvari B, Romo GM, Cruz M, Dong J, McIntire LV, Moake JL, and López JA. 2002. Ultralarge multimers of von Willebrand factor form spontaneous high- 
strength bonds with platelet glycoprotein Ib-IX complex: studies using optical tweezers. Blood. 99:3971-7.

(75) Arya M, López JA, Romo GM, Cruz MA, Kasirer-Friede A, Shattil SJ and Anvari B. 2003. Glycoprotein-Ib-IX-mediated activation of integrin allbb3: effects of receptor clustering and von Willebrand factor adhesion. Journal of Thrombosis \& Haemostasis. 1:1150-7.

(76) Shahin V, and Barrera NP. 2008. Providing unique insight into cell biology via atomic force microscopy. International Review of Cytology. 265:227-52.

(77) Lee I, and Marchant, RE. 2000. Force measurements on platelet surfaces with high spatial resolution under physiological conditions. Colloids and Surfaces B: Biointerfaces. 19:357-65.

(78) Lee I, and Marchant RE. 2003. Molecular interaction studies of hemostasis: fibrinogen ligand-human platelet receptor interactions. Ultramicroscopy. 97:34152.

(79) Hussain M, Agnihotri A, and Siedlecki CA. 1995. AFM imaging of ligand binding to platelet integrin alphaIIbbeta3 receptors reconstituted into planar lipid bilayers. Langmuir. 21:6979-86.

(80) Kuo S. 2001. Using optics to measure biological forces and mechanics. Traffic. 2:757-63.

(81) Dao M, Lim CT, and Suresh S. 2003. Mechanics of the human red blood cell deformed by optical tweezers. Journal of the Mechanics and Physics of Solids. 51:2259-80.

(82) Grier D. 2003. A revolution in optical manipulation. Nature. 424:21-7.

(83) Rand RP, and Burton AC. 1964. Mechanical properties of the red cell membrane. I. Membrane stiffness and intracellular pressure. Biophysical Journal. 4:115-135.

(84) Evans EA. 1980. Minimum energy analysis of membrane deformation applied to pipet aspiration and surface adhesion of red blood cells. Biophysical Journal. 30:265-284.

(85) Drury JL, and Dembo M. 2001. Aspiration of human neutrophils: Effects of shear thinning and cortical dissipation. Biophysical Journal. 81: 3166-77.

(86) Burris S, Smith CM, Tukey DT, Clawson CC, and White JG. 1986a. Micropipette aspiration of human platelets after exposure to aggregating agents. Arteriosclerosis. 6:321-5.

(87) Burris S, Smith II CM, Rao GHR, and White JG. 1987. Aspirin treatment reduces 
platelet resistance to deformation. Arteriosclerosis. 7:385-8.

(88) Burris S, Smith II CM, Tukey DT, Clawson CC, and White JG. 1986b. Micropipette aspiration of human platelets: Influence of rewarming on deformability of chilled cells. The Journal of Laboratory and Clinical Medicine. 107:238-43.

(89) Chien S, Sung KLP, Skalak R, Usami S, and Tozeren A. 1978. Theoretical and experimental studies on viscoelastic properties of erythrocyte membrane. Biophysical Journal. 24:463-487.

(90) Evans E, and Hochmuth RM. 1976. Membrane viscoelasticity. Biophysical Journal. $16: 1-11$

(91) Smith II C, Burris SM, Weiss DJ, and White JG. 1989. Comparison of bovine and human platelet deformability using micropipette elastimetry. American Journal of Veterinary Research. 50:34-8.

(92) Muller MR, Schima H, Engelhardt H. et al. 1993. In vitro hematological testing of rotary blood pumps: Remarks on standardization and data interpretation. Artificial Organs. 17:103-10.

(93) Holzapfel GA, and Gasser T. 2000. A new constitutive framework for arterial wall mechanics and a comparative study of material models. Journal of Elasticity. $61: 1-48$.

(94) Skalak R, Tozeren A, Zarda RP, and Chien S. 1973. Strain energy function of red blood cell membranes. Biophysical Journal. 13:245-264.

(95) Costa KD, Sim AJ, and Yin FC-P. 2006. Non-Hertzian approach to analyzing mechanical properties of endothelial cells probed by atomic force microscopy. Journal of Biomechanical Engineering. 128:176-184.

(96) Sacks MS. 2000. Biaxial mechanical evaluation of planar biological materials. Journal of Elasticity. 61:199-246.

(97) Humphrey JD, Strumpf RK, and Yin FCP. 1992. A constitutive theory for biomembranes: Application to epicardial mechanics. Journal of Biomedical Engineering. 114:461-466.

(98) Evans EA. 1973. A new material concept for the red cell membrane. Biophysical Journal. 13:926-940.

(99) McElfresh M, Baesu E, Balhorn R, Belak J, Allen MJ, and Rudd RE. 2002. Combining constitutive materials modeling with atomic force microscopy to understand the mechanical properties of living cells. The Proceedings of the National Academy of Sciences. 99:6493-6497. 
(100) Humphrey JD. Cardiovascular Solid Mechanics: Cells, Tissues, and Organs. New York: Springer, 2002.

(101) Fung YC, and Tong P. 1968. Theory of the sphering of red blood cells. Biophysical Journal. 8:175-198.

(102) Hochmuth RM, and Mohandas N. 1972. Unaxial loading of the red cell membrane. Journal of Biomechanics. 5:501-509.

(103) Golde WT, Gollobin P, and Rodriguez LL. 2005. A rapid, simple, and humane method for submandibular bleeding of mice using a lancer. Laboratory Animals (NY). 34:39-43.

(104) Gibbins JM, and Mahaut-Smith MP. 2004. Platelets and Megakaryocytes. New Jersey: Humana Press.

(105) Carmeliet P, Moons L, and Luttun A et al.. 2001. Synergism between vascular endothelial growth factor and placental growth factor contributes to angiogenesis and plasma extravasation in pathological conditions. Nature Medicine. 7:575-83.

(106) Kozek-Langenecker S. 2002. The effects of drugs used in anaesthesia on platelet membrane receptors and on platelet function. Current Drug Targets. 3:247-58.

(107) Watson SP, and Authi KS. 1996. Platelets: A Practical Approach. London: Oxford University Press.

(108) Rosenblum WI, Nelson GH, Cockrell CS, and Ellis EF. 1983. Some properties of mouse platelets. Thrombosis Research. 30:347-55.

(109) BD Vacutainer ${ }^{\circledR}$ Tube Guide. Oakville: Becton, Dickinson and Company, 2008.

(110) Kim, H-H, and Liao, JK. 2008. Translational therapeutics of dipyridamole. Arteriosclerosis, thrombosis and vascular biology. 28:s39-42.

(111) P-97 Pipette Cookbook. Novato: Sutter Instrument, 2006.

(112) Milton JS. 1999. Statistical methods in the biological and health sciences. Boston: McGraw-Hill.

(113) Hilbert D, and Cohn-Vossen S. 1999. Geometry and the imagination. New York: Chelsea.

(114) Bickford WB. 1998. Advanced mechanics of materials. California: Addison Wesley Longman, Inc. 
(115) Benham PP, Crawford RJ, and Armstrong CG. 1996. Mechanics of engineering materials. England: Pearson Education.

(116) Bronstein IN, and Semendiaev KA. 1990. Aide-mémoire de mathématiques. Paris: Eyrolles.

(117) Alenghat FJ, Nauli SM, Kolb R, Zhou J, and Ingber DE. 2004. Global cytoskeletal control of mechanotransduction in kidney epithelial cells. Experimental Cell Research. 301:23-30.

(118) Hochmuth RM. 2000. Micropipette aspiration of living cells. Journal of Biomechanics. 33:15-22.

(119) Wang N, Naruse K, Stemnovi D, Fredberg JJ, Mijailovich SM, Tolic-Nørrelykke IM, Polter T, Mannix R, and Ingber DE. 2001. Mechanical behavior in living cells consistent with the tensegrity model. Proceedings of the National Academy of Sciences. 98:7765-7770.

(120) Mody N, and King MR. 2008. Platelet adhesive dynamics. Part I: Characterization of platelets hydrodynamic collisions and wall effects. Biophysical Journal. 95: 2539-2555. 